\title{
Performance of the ATLAS track reconstruction algorithms in dense environments in LHC Run 2
}

\author{
ATLAS Collaboration ${ }^{\star}$ \\ CERN, 1211 Geneva 23, Switzerland
}

Received: 27 April 2017 / Accepted: 18 September 2017 / Published online: 11 October 2017

(c) CERN for the benefit of the ATLAS collaboration 2017. This article is an open access publication

\begin{abstract}
With the increase in energy of the Large Hadron Collider to a centre-of-mass energy of $13 \mathrm{TeV}$ for Run 2, events with dense environments, such as in the cores of highenergy jets, became a focus for new physics searches as well as measurements of the Standard Model. These environments are characterized by charged-particle separations of the order of the tracking detectors sensor granularity. Basic track quantities are compared between $3.2 \mathrm{fb}^{-1}$ of data collected by the ATLAS experiment and simulation of protonproton collisions producing high-transverse-momentum jets at a centre-of-mass energy of $13 \mathrm{TeV}$. The impact of chargedparticle separations and multiplicities on the track reconstruction performance is discussed. The track reconstruction efficiency in the cores of jets with transverse momenta between 200 and $1600 \mathrm{GeV}$ is quantified using a novel, datadriven, method. The method uses the energy loss, $\mathrm{d} E / d x$, to identify pixel clusters originating from two charged particles. Of the charged particles creating these clusters, the measured fraction that fail to be reconstructed is $0.061 \pm 0.006$ (stat.) \pm 0.014 (syst.) and $0.093 \pm 0.017$ (stat.) \pm 0.021 (syst.) for jet transverse momenta of $200-400 \mathrm{GeV}$ and $1400-1600 \mathrm{GeV}$, respectively.
\end{abstract}

\section{Introduction}

The Large Hadron Collider (LHC) entered a new energy regime in 2015, at the start of Run 2, with proton-proton collisions at a centre-of-mass energy of $13 \mathrm{TeV}$. Events with $\mathrm{TeV}$-scale jets showering in the detectors, or $\tau$-leptons and $b$-hadrons that pass through multiple active layers of material, now occur at high enough rates to be studied in detail. These signatures also occur in potential new physics scenarios including massive new resonances decaying to highly boosted bosons or top quarks whose decay products are often reconstructed as a single jet [1]. In the cores of highly

^e-mail: atlas.publications@ @ern.ch energetic hadronic jets and $\tau$-leptons, the average separation between highly collimated charged particles is comparable to the granularity of individual sensors of the inner detector. This can create confusion within the algorithms used to reconstruct charged-particle trajectories, or tracks. Therefore, without careful consideration, the track reconstruction efficiency in these dense environments is limited, resulting in difficulties in identifying long-lived $b$-hadrons and hadronic $\tau$-decays, or in calibrating the energy and mass of jets. To prevent losses in efficiency, to increase the possibility of discovering new phenomena and to allow more detailed measurements of the newly opened kinematic regime, a dedicated optimization for dense environments was performed and deployed in the ATLAS [2] reconstruction for the start of Run 2. This updated reconstruction provides superior physics performance, reduces the required computing resources, and is now the default used by ATLAS.

This paper first describes the ATLAS detector (Sect. 2). Then, a general overview of the track reconstruction algorithm (Sect. 3) is given, focusing on the performance of charged-particle reconstruction in dense environments at the start of Run 2. The data set utilized is described in Sect. 4. The quality of the expected performance is evaluated in dedicated single-particle and dijet simulation samples (Sect. 5), and comparisons between simulation and data are performed in events with energetic jets. Extending these mainly Monte Carlo (MC) simulation-based studies, a fully data-driven method is introduced in Sect. 6 which probes the fraction of tracks lost in reconstruction, due to the high density and collimation of charged particles in high-transverse-momentum ${ }^{1}$ $\left(p_{\mathrm{T}}\right)$ jets. This is achieved by using the ionization energy loss $(\mathrm{d} E / d x)$ in the pixel detector.

\footnotetext{
1 ATLAS uses a right-handed coordinate system with its origin at the nominal interaction point (IP) in the centre of the detector and the $z$-axis along the beam pipe. The $x$-axis points from the IP to the centre of the LHC ring, and the $y$-axis points upwards. Cylindrical coordinates $(r, \phi)$ are used in the transverse plane, $\phi$ being the azimuthal angle around the $z$-axis. The pseudorapidty is defined in terms of the polar angle $\theta$ as $\eta=$ $-\ln \tan (\theta / 2)$. Angular distance is defined as $\Delta R \equiv \sqrt{(\Delta \eta)^{2}+(\Delta \phi)^{2}}$.
} 


\section{The ATLAS detector}

The ATLAS experiment, a multipurpose particle detector at the LHC, covers almost the entire solid angle around the collision point, and consists of an inner detector (ID) tracking system surrounded by a thin superconducting solenoid magnet producing a $2 \mathrm{~T}$ axial magnetic field, electromagnetic and hadronic calorimeters, and a muon spectrometer incorporating three large toroid magnet assemblies.

The ID, shown in Fig. 1, provides position measurements for charged particles in the range $|\eta|<2.5$ by combining information from three subdetectors. It consists of a cylindrical barrel region (full coverage for $|\eta| \lesssim 1.5$ ) arranged around the beam pipe, and two end-caps. Disks in the endcap region are placed perpendicular to the beam axis, covering $1.5<|\eta|<2.5$. Starting from the interaction point, the high-granularity silicon pixel detector segmented in $r-\phi$ and $z$ (including the new innermost layer, the insertable B-layer (IBL) [3,4] added for Run 2) covers the vertex region and typically provides four measurements per track. The IBL has a mean radius of $33 \mathrm{~mm}$ and a typical IBL pixel has a size of $50 \mu \mathrm{m}$ by $250 \mu \mathrm{m}$ in the transverse and longitudinal directions with a sensor thickness of $200 \mu \mathrm{m}$. For the remaining three layers of the pixel system, located at mean radii of 50.5,
88.5, and $122.5 \mathrm{~mm}$ respectively, a typical pixel has a size of $50 \mu \mathrm{m}$ by $400 \mu \mathrm{m}$ in the transverse and longitudinal directions with a thickness of $250 \mu \mathrm{m}$. The pixel layer at a radius of $50.5 \mathrm{~mm}$ is referred to as the B-layer in this paper. The coverage in the end-cap region is enhanced by three disks on either side of the interaction point. The pixel detectors measure the charge collected in each individual pixel using the time over threshold (ToT) [5]. ToT is the time the pulse exceeds a given threshold and is proportional to the deposited energy.

Outside the pixel volume, the barrel of the silicon microstrip detector (SCT) consists of four double strip layers at radii of $299-514 \mathrm{~mm}$, complemented by nine disks in each of the end-caps. A typical strip of a barrel SCT sensor has a length of $126 \mathrm{~mm}$ and a pitch of $80 \mu \mathrm{m}$. On each layer, the strips are parallel to the beam direction on one side and at a stereo angle of $40 \mathrm{mrad}$ on the other. The information from the two sides of each layer can be combined to provide an average of four three-dimensional measurements per track. The SCT sensors are connected to binary read-out chips, which do not provide information about the collected charge. The silicon detectors are complemented by the transition radiation tracker (TRT) [6], which extends track reconstruction radially up to a radius of $1082 \mathrm{~mm}$ for

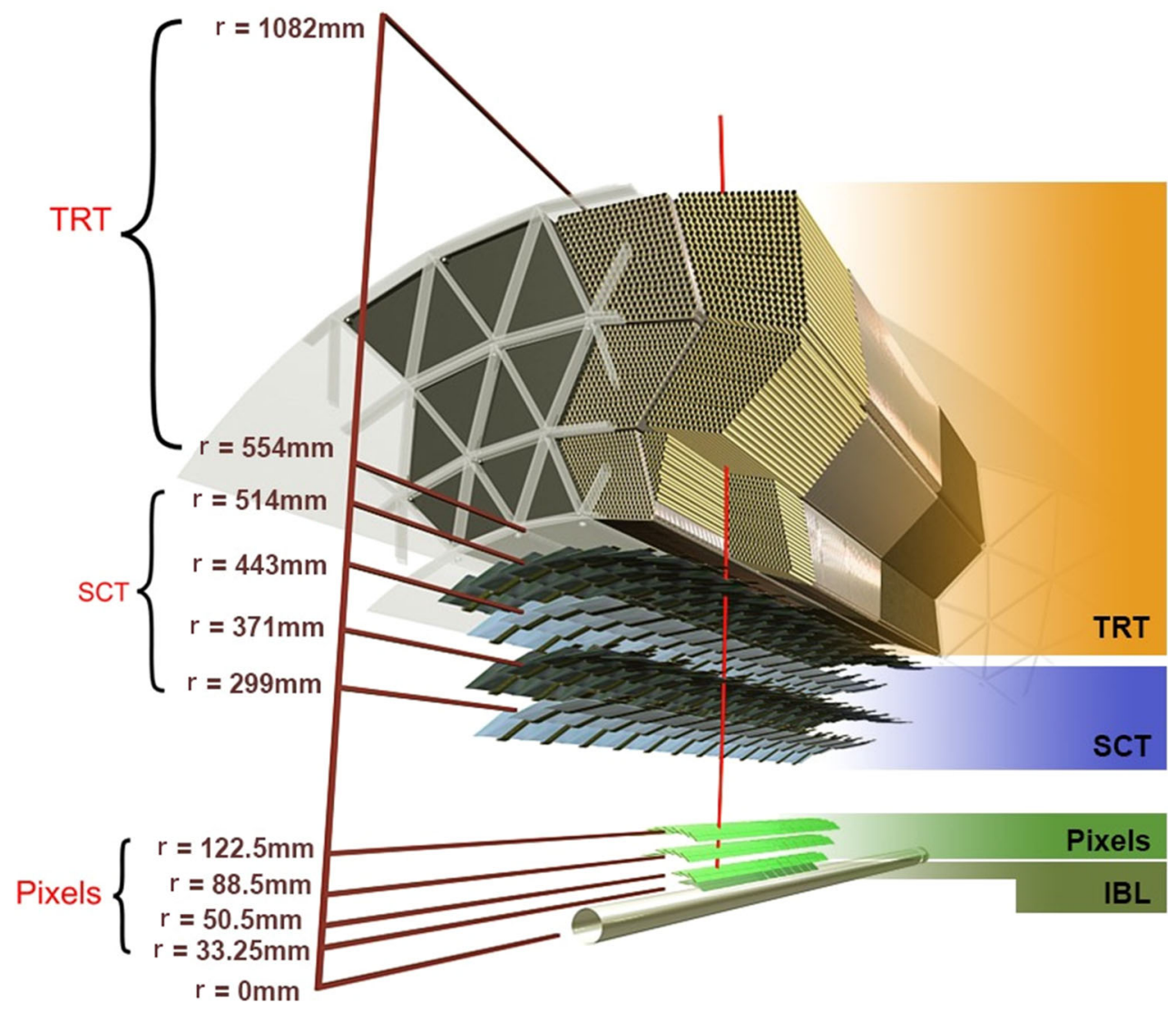

Fig. 1 Sketch of the barrel region of the ATLAS inner detector 
charged particles within $|\eta|=2.0$ while providing $r-\phi$ information. The raw timing information from its straw tubes is translated into calibrated drift circles that are matched to track candidates reconstructed from the silicon detectors [6].

The solenoid is surrounded by sampling calorimeters. Calorimetry is provided by three distinct detectors outside the ID volume. A lead/liquid-argon sampling electromagnetic calorimeter is split into barrel $(|\eta|<1.5)$ and end-cap $(1.5<|\eta|<3.2)$ sections. A steel/scintillator-tile hadronic calorimeter covers the barrel region $(|\eta|<1.7)$ and two end-cap copper/liquid-argon sections extend to higher pseudorapidity $(1.5<|\eta|<3.2)$. Finally, the forward region

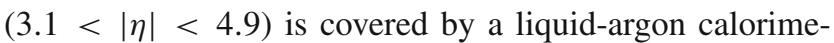
ter with a copper (tungsten) absorber in the electromagnetic (hadronic) section. In the outermost part, air-core toroids provide the magnetic field for the muon spectrometer. It consists of three layers of gaseous detectors: monitored drift tubes and cathode strip chambers for muon identification and momentum measurements for $|\eta|<2.7$, and resistive-plate and thingap chambers for online event selection up to $|\eta|=2.4$. A two-level trigger system, custom hardware followed by a software-based level, is used for online event selection and to reduce the event rate to about $1 \mathrm{kHz}$ for offline reconstruction and storage.

\section{ATLAS track reconstruction}

The following provides an overview of primary-track reconstruction in the pixel and SCT detectors. After cluster creation, the primary-track reconstruction algorithm utilizes iterative track-finding seeded from combinations of silicon detector measurements, while additional methods are employed to recover non-prompt tracks. A staged patternrecognition approach is used: a loose track candidate search, which allows a number of combinatorial track candidates, is followed by a stringent ambiguity-solver that compares and rates the individual tracks by assigning a relative track score to each track. This follows current approaches to track reconstruction first introduced in Ref. [7]. Further details, including a description of TRT track extensions, can be found in Ref. [8].

\subsection{Clusterization}

Charged-particle reconstruction in the pixel and SCT detectors begins by assembling clusters from the raw measurements. A connected component analysis (CCA) [9] groups pixels and strips in a given sensor, where the deposited energy yields a charge above threshold, with a common edge or corner into clusters. From these clusters, three-dimensional measurements referred to as space-points are created. They represent the point where the charged particle traversed the active material of the ID. In the pixel detector, each cluster equates to one space-point, while in the SCT, clusters from both sides of a strip layer must be combined to obtain a threedimensional measurement.

The charge in a pixel sensor is often collected on multiple adjacent pixels. In the data set described in Sect. 4, the average size of pixel clusters in the barrel is about two pixels in the $r-\phi$ plane and from one to three pixels in the longitudinal direction increasing with $\eta$. The total charge is proportional to the path length in the sensor and thus dependent on the incident angle of the particle. The particle's intersection point with the sensor is determined from the pixels contributing to the cluster using a linear approximation refined with a charge interpolation technique [10]. In dense environments, the spatial separation between charged particles traversing the sensor is only a few pixels, and the CCA algorithm, at times, reconstructs only one cluster which includes energy deposits from multiple particles. Identifying such clusters reliably and quickly is paramount for an efficient chargedparticle reconstruction in dense environments.

It is useful to introduce the several classes of clusters identified by either the "truth information", only available in simulation and referring to information at $\mathrm{MC}$ generator level, or reconstructed quantities in both collision data and $\mathrm{MC}$ simulation. Clusters created by charge deposits from one particle are called single-particle clusters. Clusters created by charge deposits from multiple particles are called merged clusters. These definitions rely on truth information and both cases are illustrated in Fig. 2. Based on information available in the track reconstruction algorithm described below, clusters which are compatible with a merged cluster can be identified. These are labelled identified as merged. Ideally, all clusters identified as merged are, in fact, merged clusters, and all merged clusters are identified as merged. Shared clusters are those which are used in multiple reconstructed tracks but are not sufficiently compatible with the properties of a merged cluster to be identified as merged by the reconstruction. $\mathrm{Mul}$ tiply used clusters - clusters used by multiple tracks - are either identified as merged or shared but not both.

\subsection{Iterative combinatorial track finding}

Track seeds are formed from sets of three space-points. This approach maximizes the possible number of combinations while still allowing a first crude momentum estimate. The impact parameters of a track seed, with respect to the centre of the interaction region, are estimated by assuming a perfect helical trajectory in a uniform magnetic field.

The purity, or fraction of seeds that result in good-quality tracks, varies significantly depending on which subdetector(s) recorded the space-points used in the seed. Therefore, seed types are considered starting with SCT-only, then 


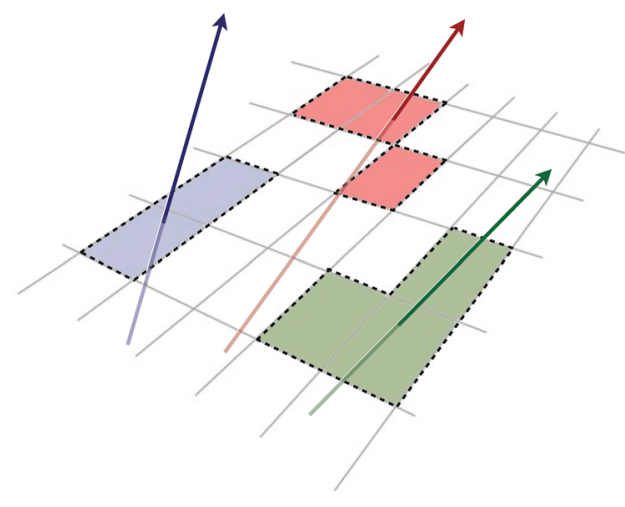

(a) Single-particle pixel clusters

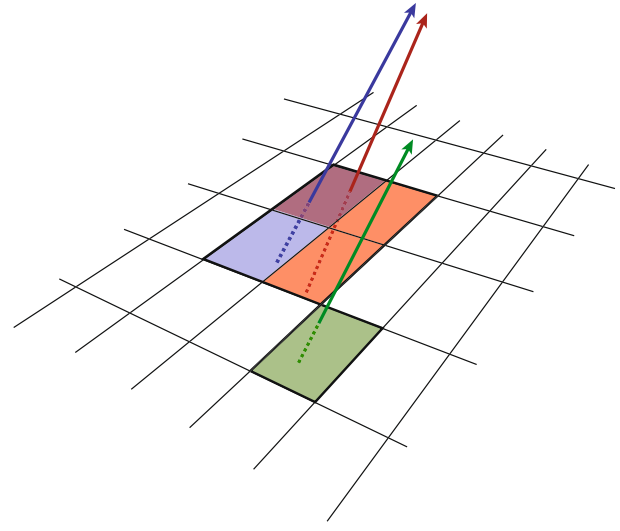

(b) Merged pixel cluster

Fig. 2 Illustration of a single-particle pixel clusters on a pixel sensor and $\mathbf{b}$ a merged pixel cluster due to very collimated charged particles. Different colours represent energy deposits from different charged particles traversing the sensor and the particles trajectories are shown as arrows

pixel-only and finally mixed-detector seeds, representing the order of purity. A number of criteria are placed on the seeds to maximize purity: first and foremost seed-type-dependent momentum and impact parameter requirements. Also, the use of space-points in multiple seeds is carefully controlled. Purity is further improved by requiring that one additional space-point is compatible with the particle's trajectory estimated from the seed. A combinatorial Kalman filter [11] is then used to build track candidates from the chosen seeds by incorporating additional space-points from the remaining layers of the pixel and SCT detectors which are compatible with the preliminary trajectory. The filter creates multiple track candidates per seed if more than one compatible spacepoint extension exists on the same layer.

These criteria result in a very high efficiency for reconstructing primary particles (for example, the muon reconstruction efficiency is greater than 99\% [12]) and the removal of tracks created from purely random collections of spacepoints. Suppressing such purely combinatorial tracks is essential in order to remain within the available CPU budget for event reconstruction. From approximately 13 space-point combinations created for an isolated charged particle traversing the entire ID, the time-intensive combinatorial Kalman filter is, on average, called in its entirety 1.1 times. As all realistic combinations of space-points have been made, there are a number of track candidates where space-points overlap, or have been incorrectly assigned. This necessitates an ambiguity-solving stage.

\subsection{Track candidates and ambiguity solving}

In the ambiguity solver, track candidates considered to create the reconstructed track collection are processed individually in descending order of a track score, favouring tracks with a higher score. This design relies on having an appropriate track score definition that puts tracks into an order that scores more highly the candidates likely to correctly represent the trajectory of a charged primary particle.

The method used to determine the track score, discussed in the following, applies a robust approach based largely on simple measures of the track quality. Clusters assigned to a track increase the track score according to configurable weight fractions reflecting the intrinsic resolutions and expected cluster multiplicities in the different subdetectors. Holes ${ }^{2}$ reduce the score. The $\chi^{2}$ of the track fit is also considered to penalize candidates with a poor fit. Finally, the logarithm of the track momentum is considered to promote energetic tracks and suppress the larger number of tracks with incorrectly assigned clusters, which typically have a low $p_{\mathrm{T}}$.

After the track scores have been calculated, the ambiguity solver deals with clusters assigned to multiple track candidates. Clusters compatible with multiple track candidates are a natural consequence of having merged clusters in dense environments. High reconstruction efficiency is facilitated by the identification of merged clusters, as explained in Sect. 3.4. However, shared clusters, clusters used in multiple track candidates which are not identified as merged, must be limited as they are a strong indicator of incorrect assignments.

To count shared clusters, a track candidate is only compared to those tracks previously accepted by the ambiguity solver. Clusters can be shared by no more than two tracks, giving preference to tracks processed first in the ambiguity

\footnotetext{
${ }^{2}$ Holes are defined as intersections of the reconstructed track trajectory with a sensitive detector element that does not contain a matching cluster. These are estimated by following closely the track trajectory and comparing, within the uncertainties, the intersected sensors with the clusters on the track. Inactive sensors or regions, such as edge areas on the silicon sensors, are excluded from the hole definition.
} 


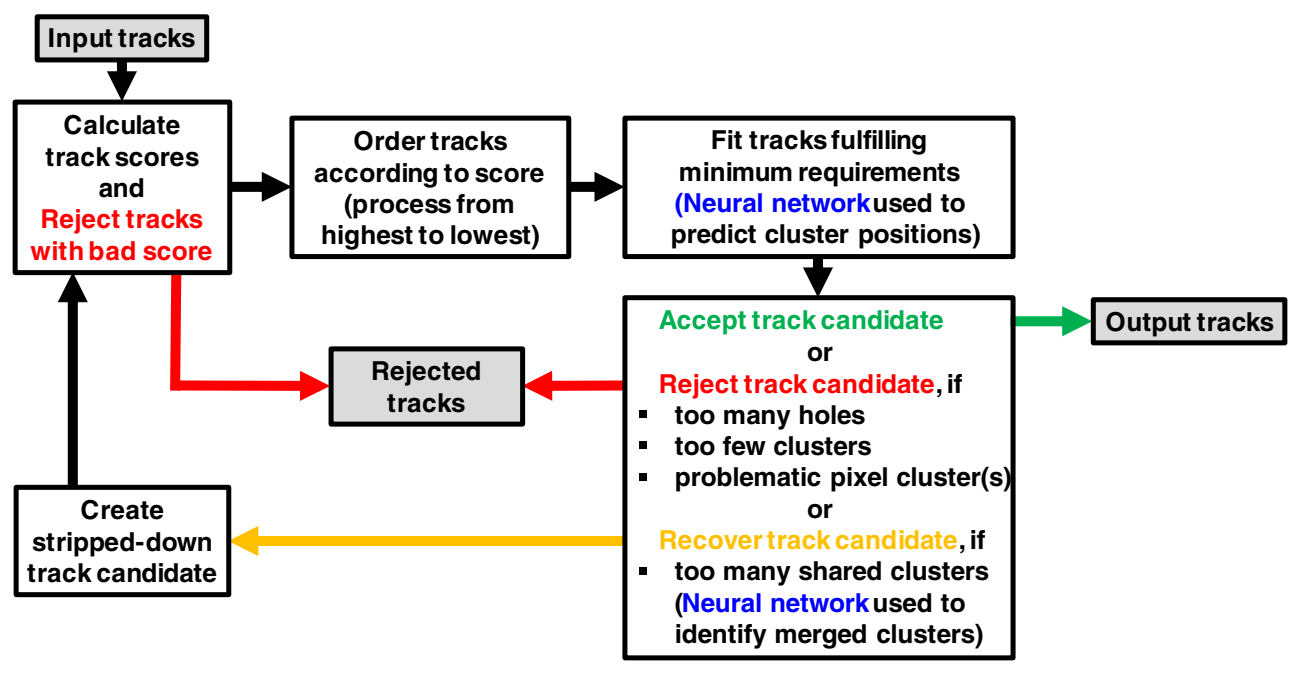

Fig. 3 Sketch of the flow of tracks through the ambiguity solver

solver. Also, a track can have no more than two shared clusters. A cluster is removed from a track candidate if it causes either the candidate or an accepted track to not meet the shared-cluster criterion. The track candidate is then scored again and returned to the ordered list of remaining candidates. Track candidates are rejected by the ambiguity solver if they fail to meet any of the following basic quality criteria:

- $p_{\mathrm{T}}>400 \mathrm{MeV}$,

- $|\eta|<2.5$,

- Minimum of 7 pixel and SCT clusters (12 are expected),

- Maximum of either one shared pixel cluster or two shared SCT clusters on the same layer,

- Not more than two holes in the combined pixel and SCT detectors,

- Not more than one hole in the pixel detector,

- $\left|d_{0}^{\mathrm{BL}}\right|<2.0 \mathrm{~mm}$,

- $\left|z_{0}^{\mathrm{BL}} \sin \theta\right|<3.0 \mathrm{~mm}$,

where $d_{0}^{\mathrm{BL}}$ is the transverse impact parameter calculated with respect to the measured beam-line position, $z_{0}^{\mathrm{BL}}$ is the longitudinal difference along the beam line between the point where $d_{0}^{\mathrm{BL}}$ is measured and the primary vertex, ${ }^{3}$ and $\theta$ is the polar angle of the track. In the remainder of the paper, all studied tracks fulfil these requirement. A simplified flow of track candidates through the ambiguity solver is shown in Fig. 3.

\footnotetext{
3 All events considered in this analysis are required to have at least one reconstructed primary vertex with at least two associated tracks [13]. Only tracks compatible with the primary vertex having the highest sum of the squared transverse momenta of its associated tracks are considered.
}

\subsection{Neural-network pixel clustering}

To aid the ambiguity solver and minimize the loss of efficiency due to limitations on the number of shared clusters per track, an artificial neural network (NN) trained to identify merged clusters is used. The measured charge, which is proportional to the deposited energy, and relative position of pixels in the cluster can be used to identify merged clusters. Additional information about the particle's incident angle, provided from the track candidate, significantly improves the NN's performance [14]. For merged clusters created by two charged particles, the $\mathrm{NN}$ identification efficiency of this cluster as being created by two particles is about $90 \%$. Merged clusters created by three charged particles are identified as such with an efficiency of $85 \%$. Only a few percent of single particle clusters are incorrectly identified as a twoparticle merged cluster and a negligible amount are identified as three-particle merged clusters. The NN is not able to distinguish clusters from exactly three and more than three charged particles. It is not possible for the NN to separate the energy deposits of each charged particle in an identified merged cluster and subsequently divide it into multiple clusters. Unlike the Run-1 reconstruction algorithm [8], the NN is consulted only when a cluster is used in multiple track candidates largely mitigating the impact of misidentification of merged clusters by the NN.

The inherent randomness of charged-particle interactions with thin silicon layers prevents the NN from performing perfectly. For example, the emission of $\delta$-rays causes difficulties as they can lead to bigger clusters and larger energy deposits than expected from a single particle. These inefficiencies can be mitigated by correlating information from consecutive layers of the pixel detector. In general, the separation between collimated charged particles increases as they 
travel outward through the ID. Therefore, if a pair of tracks uses a merged cluster on a given layer, then the inner layer is likely to contain a merged cluster as well. Furthermore, both clusters should be used by the same track candidates in this logic.

In summary, a cluster can be identified as merged in two ways. Either it is used by multiple track candidates and the NN identifies it as a merged cluster, or if two track candidates compete for clusters on two consecutive layers, the cluster on the inner layer is identified as merged if the cluster on the outer layer is identified as merged. Clusters identified as merged are used by the competing track candidates without penalty. Clusters which are not identified as merged, shared clusters, can still be used in multiple tracks but with the penalty described in Sect. 3.3.

\subsection{Track fit}

For track candidates fulfiling the requirements listed in Sect. 3.3, a high-resolution fit is performed using all available information. Fitted tracks which pass through the ambiguity solver without modification are added to the final track collection. Delaying the track fit until this stage minimizes the number of times the fitter is called, which is advantageous as it is a relatively CPU-intensive process.

For the high-resolution track fits, the position and uncertainty of each cluster is determined by additional NNs [14]. They predict the positions where the charged particles intersected the sensor based on the same input to the $\mathrm{NN}$ described in Sect. 3.4. The predicted number of charged particles which created the cluster determines the number of particle intersections the additional NNs predict. This decreases the discrepancy between the reconstructed cluster position and the cluster's fitted track position at the detector surface, especially for merged clusters, resulting in more precise track parameters.

\section{Data and Monte Carlo samples}

Data from proton-proton collisions at $\sqrt{s}=13 \mathrm{TeV}$, collected during 2015 and corresponding to an integrated luminosity of $3.2 \mathrm{fb}^{-1}$, are used in this paper. Events are selected using triggers requiring a single jet above various $p_{\mathrm{T}}$ thresholds. The minimum jet trigger $p_{\mathrm{T}}$ threshold is $100 \mathrm{GeV}$. The numbers of events selected by the triggers were reduced by a factor depending on the instantaneous luminosity and the jet $p_{\mathrm{T}}$ threshold. This suppresses the number of low- $p_{\mathrm{T}}$ jets while keeping all events with at least one jet with $p_{\mathrm{T}}$ $>450 \mathrm{GeV}$. Standard ATLAS data-quality requirements are applied to all data sets, ensuring all detectors were operational.
The data are compared to a leading-order dijet MC sample generated with PYTHIA 8.186 [15] with the A14 tuned parameter set [16] and the NNPDF2.3LO parton distribution function (PDF) set [17]. MC samples generated with Herwig++2.7.1 [18], and SHERPA 2.1 [19] are also studied. For Herwig++, the UEEE5 tuned parameter set is used with the CTEQ6L1 PDF set [20] and for SHERPA, parameters corresponding to the CT10 PDF set [21] are used. The ATLAS detector response is fully simulated [22] using the GEANT 4 framework [23]. The average number of protonproton interactions per bunch crossing (pile-up) was approximately 15 during the 2015 data-taking period. The expected contribution from additional proton-proton interactions is accounted for by overlaying minimum-bias events simulated with PYTHIA 8. The MC samples are reweighted to match the distribution of the number of interactions per bunch crossing and then reweighted to the inclusive jet- $p_{\mathrm{T}}$ spectrum observed in collision data. In dense environments, the impact of pile-up on the track reconstruction performance is small. The change in tracking efficiency considering only one interaction per bunch crossing to an average pile-up of 40 in the dijet MC sample for jets with a $p_{\mathrm{T}}$ above $200 \mathrm{GeV}$ is below $0.3 \%$.

In order to perform detailed simulation-based studies on event topologies with highly collimated particles, four large MC samples, with a single particle decaying into a set of nearby charged particles, are employed. The initial particles have different lifetimes and decay multiplicities, and are generated with a uniform transverse momentum spectrum from 10 to $1 \mathrm{TeV}$ within $|\eta|$ of 1.0. Topologies with two highly collimated tracks are studied in a simulated $\rho \rightarrow \pi^{+} \pi^{-}$sample. Simulated decays of a single $\tau$-lepton to three charged hadrons $\left(\tau^{ \pm} \rightarrow \pi^{+} \pi^{-} \pi^{ \pm} \nu_{\tau}\right)$ are used to study topologies with three charged particles. To study the performance in topologies with higher charged-particle multiplicities, two additional samples are created; a sample containing all decays of a $B^{0}$ into multiple particles and a $\tau$-lepton decaying to a final state including five charged hadrons.

\section{Track reconstruction performance in dense environments}

This section first compares basic properties of tracks inside jets in data with those in simulated dijet samples (Sect. 5.2). Using truth-based quantities, Sect. 5.3 studies single-particle decays with collimated decay products. These relatively simple topologies allow the behaviour of the track reconstruction to be studied as a function of the momentum of the initial particle, and the spatial separation between the tracks. Section 5.4 presents analogous results, but derived from a dijet MC sample of high- $p_{\mathrm{T}}$ jets. 


\subsection{Classification}

In simulation, tracks are classified using a truth-matching probability. It is the ratio of the weighted number of measurements originating, at least in part, from the same simulated particle, to the weighted number of all measurements used in a track. A subdetector-specific weight of ten for measurements in the pixel detector, five for the SCT and one for the TRT is used. These weights reflect the average number of expected measurements in each subdetector. A properly reconstructed track is required to have a truth-matching probability above 0.5 . Such a requirement is imposed for all reconstruction efficiencies presented in this paper.

Fake tracks are those which have a truth-matching probability below 0.5 . Due to the careful pruning of seeds, the majority of reconstructed fake tracks are from the misallocation of clusters from other particles to a track and not purely random combinations of clusters. The track reconstruction procedure described in Sect. 3 results in a negligible number of fake tracks in dense environments. For jets with a $p_{\mathrm{T}}$ above $200 \mathrm{GeV}$ in the dijet MC sample described in Sect. 4, the fraction of fake tracks is below $0.5 \%$. From only one $p p$ interaction per bunch crossing to an average pile-up of 40 , this fraction increases by about $0.5 \%$, still making it negligible. Consequently, fake tracks are not discussed in further detail.

Jets are reconstructed from topological clusters [24] of energy deposits in the calorimeter using the anti- $k_{t}$ algorithm [25] with a radius parameter $R=0.4$ and are selected requiring a minimum jet $p_{\mathrm{T}}$ of $200 \mathrm{GeV}$ and $\left|\eta^{\text {jet }}\right|<2.5$. Jets are corrected for the effects of non-compensating response in the calorimeter and inactive material by using energyand $\eta$-dependent calibration factors, based on MC simulation and $p p$ collision data. Additional corrections are applied to reduce the dependence of the jet energy measurement on the longitudinal and transverse structure of the jets and also to correct for jets that are not fully contained in the calorimeter [26].

\subsection{Data and MC simulation comparison}

This section gives an overview of basic properties of tracks inside jets. Data and MC simulation comparisons establish fair agreement between the two.

The average number of tracks per unit of angular area versus the angular distance from the jet axis in data and MC events is compared in Fig. 4. The charged-particle density in jets increases linearly with the logarithm of the jet momentum, which reflects the average number of tracks inside the jet. Moreover, most tracks are located within an angular distance of 0.05 from the jet axis. Jets in data tend to have a slightly wider distribution of reconstructed charged particles than those in simulation.

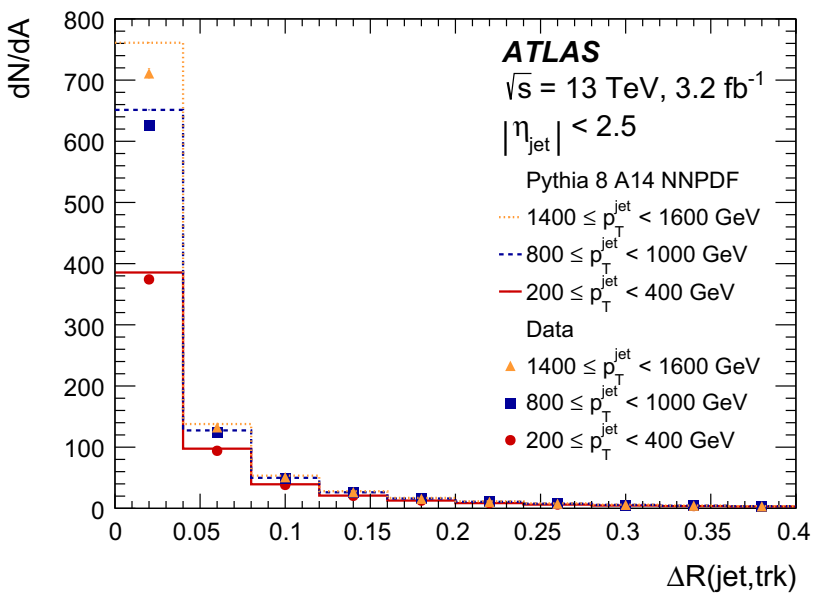

Fig. 4 The average number of primary tracks per unit of angular area as a function of the angular distance from the jet axis. Data (markers) and dijet MC (lines) samples are compared in bins of jet $p_{\mathrm{T}}$ showing the high density in the cores of energetic jets

Due to the large number of collimated charged particles the number of multiply used clusters rises steeply at small distances to the jet axis. Figure 5 shows the number of pixel clusters that are identified as merged and the number of shared pixel clusters on the track for data and MC simulation versus the angular distance from the jet axis. The average number of shared pixel clusters remains relatively low compared to the number of clusters identified as merged, down to the smallest distances, because the reconstruction algorithm identifies merged clusters with high efficiency, and these consequently are not counted as shared. MC simulation and data show reasonable agreement in the individual bins of jet $p_{\mathrm{T}}$.

Inefficiencies in the identification and treatment of merged clusters affect the number of IBL clusters on tracks in dense environments. Figure 6 shows the average number of IBL clusters on the track, for data and MC simulation versus the angular distance from the jet axis. For small distances the number of IBL clusters shows a drop, explained by a residual inefficiency in assigning clusters to the appropriate track. MC simulation and data agree within expectations in each of the individual jet $p_{\mathrm{T}}$ bins. The overall lower average number of IBL clusters on track in data is due to a not fully functional IBL detector module, which is not correctly considered in MC simulation.

Although the SCT sensors are located at much higher radii than the pixel sensors, the expected number of shared clusters is considerably larger than for the pixels as shown in Fig. 7. This is due to the coarser segmentation of the SCT strips in one dimension and the lack of charge information hindering the identification of merged SCT clusters. The average number of shared SCT clusters decreases with the angular distance from the jet axis, correlated with the decrease in charged-particle density visible in Fig. 4 for data and MC 


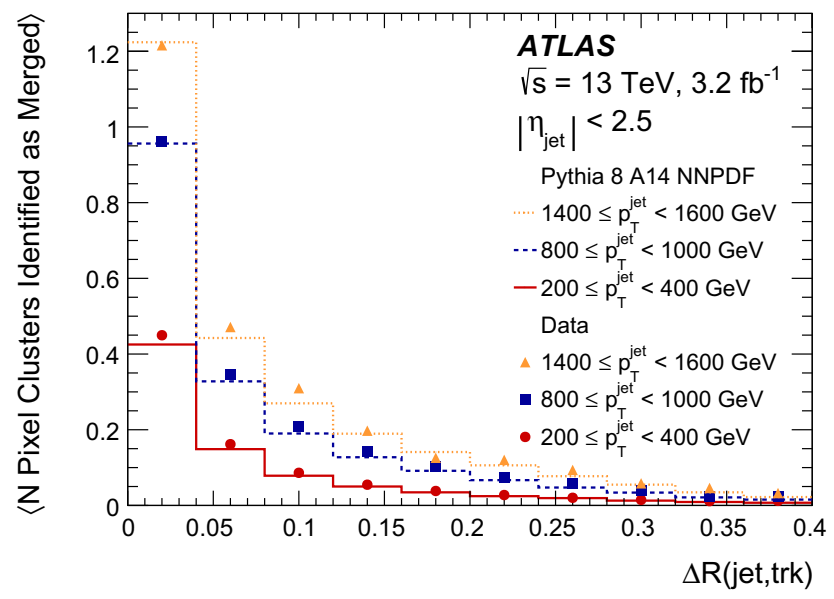

(a) Pixel clusters identified as merged

Fig. 5 The average number of a pixel clusters identified as merged and $\mathbf{b}$ shared pixel clusters on primary tracks (with a production vertex before the IBL) are shown as a function of the angular distance of the track from the jet axis. Data (markers) and dijet MC (lines) samples

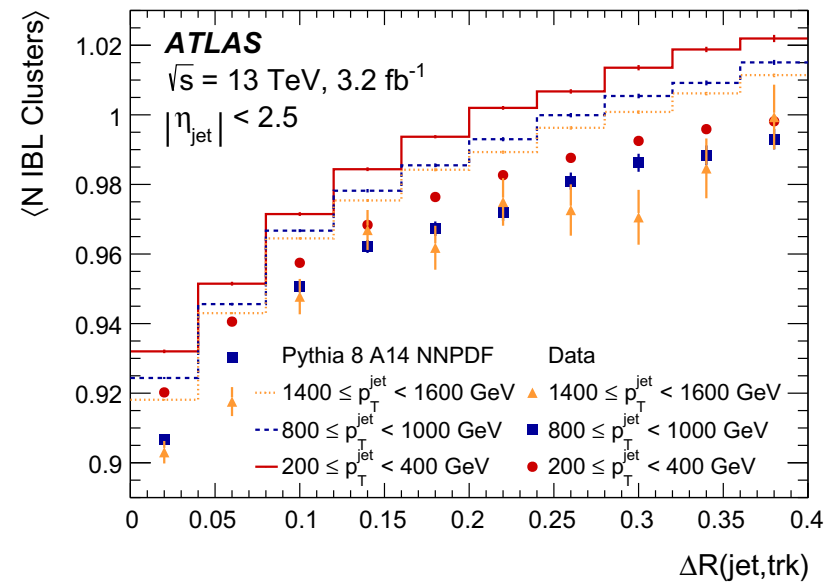

Fig. 6 The average number of IBL clusters on primary tracks (with a production vertex before the IBL) shown as a function of the angular distance of the track from the jet axis. Data (markers) and dijet MC (lines) samples are compared in bins of jet $p_{\mathrm{T}}$ showing a slight drop at small distances explained by a residual cluster-to-track assignment inefficiency

simulation. In the studied jet- $p_{\mathrm{T}}$ range, the average number of SCT clusters on tracks is approximately 7.7 with little variation with respect to angular distances from the jet axis. The MC simulation agrees within expectations with data in the individual bins of jet $p_{\mathrm{T}}$.

\subsection{Performance for collimated tracks}

Quantities such as cluster assignment and track reconstruction efficiencies can be studied using truth information from simulation to elucidate the track reconstruction behaviour

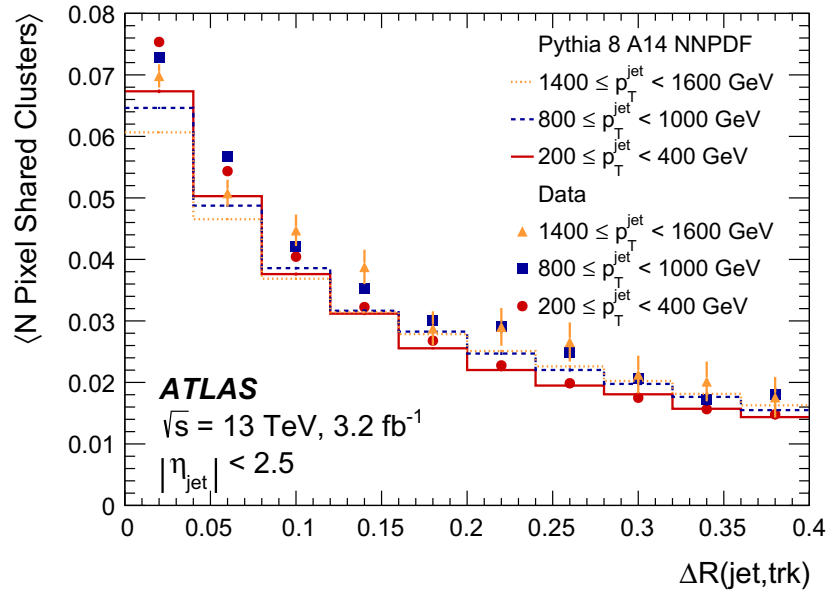

(b) Shared pixel clusters

are compared in bins of jet $p_{\mathrm{T}}$. The rise in both populations at small distances from the jet axis is expected due to the increasingly dense environment

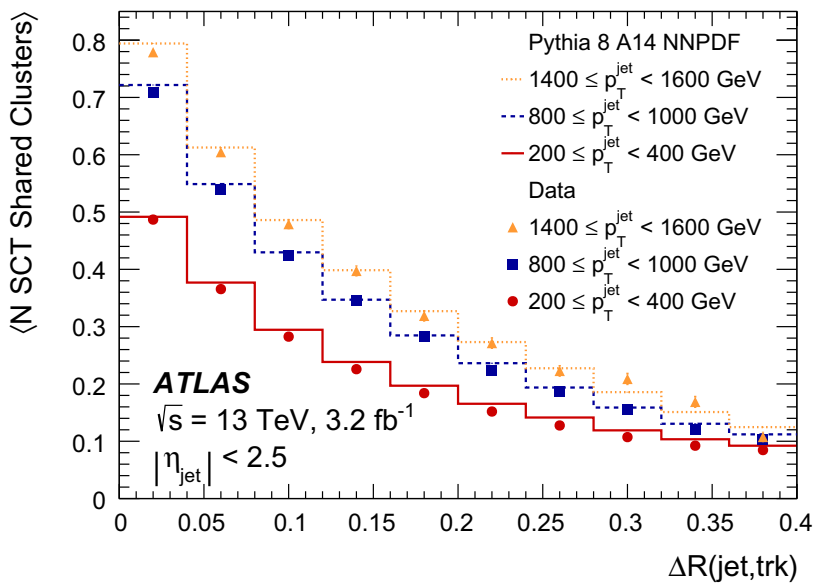

Fig. 7 The average number of shared SCT clusters for primary tracks with a production vertex before the IBL is shown as a function of the angular distance of the track from the jet axis. Data (markers) and dijet MC (lines) samples are compared in bins of jet $p_{\mathrm{T}}$. Due to the lack of charge information and the coarse sensor dimensions, the clusters cannot be readily identified as merged

in the presence of highly collimated charged particles. This section utilizes the single-particle samples described in Sect. 4. Figure 8 shows how the minimum separation between charged particles at the IBL sensor surfaces evolves with the initial particle's $p_{\mathrm{T}}$. For the same $p_{\mathrm{T}}$, the density of the decay products may differ significantly: the lighter the initial particle, or the higher the multiplicity of its decay products, the smaller the distance. The degradation of the track reconstruction performance is mainly driven by the distance between charged particles and the charged-particle multiplicity in their vicinity. The results presented hereafter are 


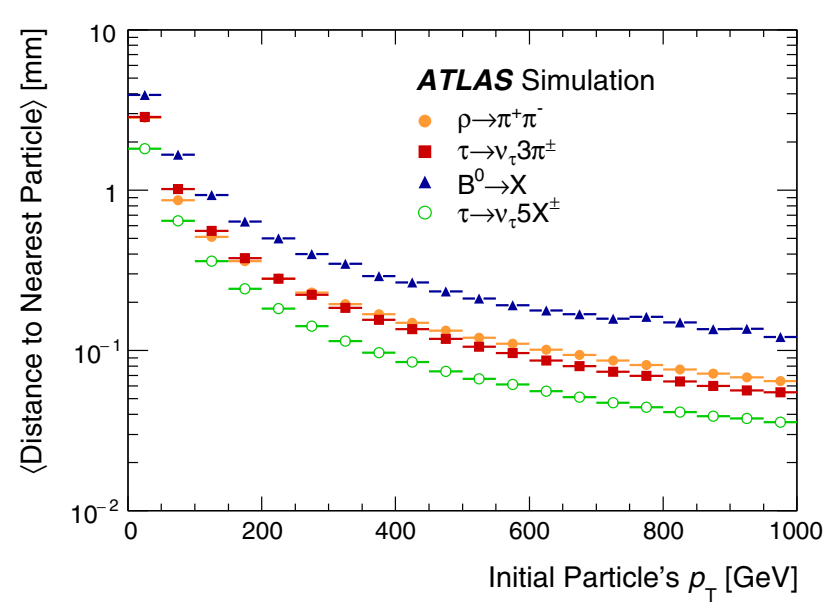

Fig. 8 A comparison of the average minimum distance between charged decay products at the IBL sensor surfaces as a function of initial particle's $p_{\mathrm{T}}$ for single-particle samples

therefore representative of the reconstruction performance in many physics processes, provided these parameters are known. Throughout this section, unless otherwise noted, it is required that all charged particles are created before the IBL (production radius smaller than $29 \mathrm{~mm}$ ) in all figures shown.

The average number of merged clusters is compared to the average number of clusters identified as merged in Fig. 9 for the single $\rho$ and three-prong $\tau$ samples. The average charged-particle separation decreases with increasing initialparticle $p_{\mathrm{T}}$ leading to more merged pixel clusters as shown in the points labelled Ideal. The average numbers of both the merged clusters and the clusters identified as merged fall to zero at the lowest initial-particle $p_{\mathrm{T}}$, confirming a low rate

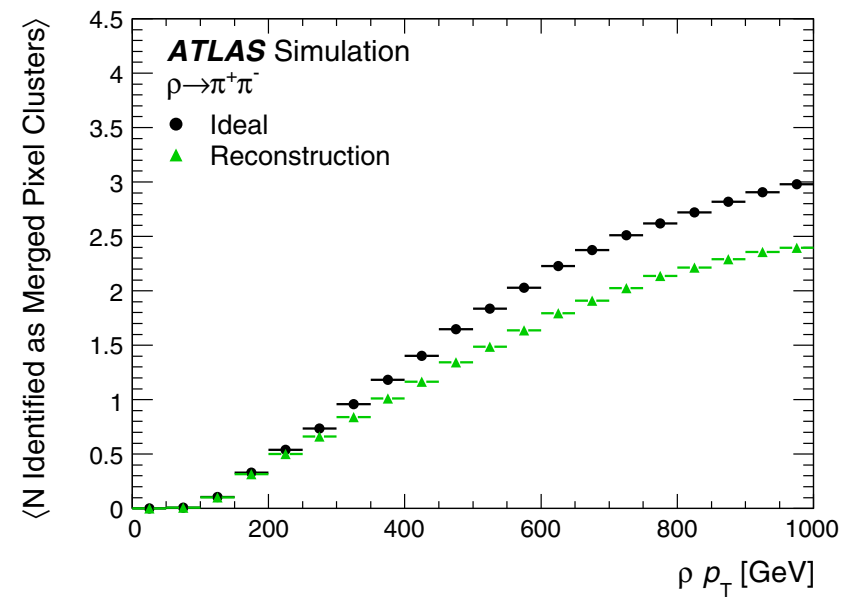

(a) $\rho \rightarrow \pi^{+} \pi^{-}$sample

Fig. 9 A comparison of the average number of merged pixel clusters expected for truth particles from simulation and pixel clusters identified as merged used in reconstructed tracks is shown as a function of the $\rho$ and three-prong $\tau\left(\tau \rightarrow \pi^{+} \pi^{-} \pi^{ \pm} \nu_{\tau}\right)$ transverse momentum. Ideal of false-positives. Both grow at a similar rate with increasing initial-particle $p_{\mathrm{T}}$. The residual inefficiency of the pixel $\mathrm{NN}$ is apparent in a lower number of clusters identified as merged compared to the ideal number of merged clusters at high initial-particle $p_{\mathrm{T}}$. The reconstruction performance correlates directly with the multiplicity and distances at a given initial-particle $p_{\mathrm{T}}$ shown in Fig. 8.

Merged clusters failing identification can result in shared clusters, which (as explained in Sect. 3.3) need to be limited. To study possible inefficiencies of the reconstruction algorithm, the cluster assignment efficiency is shown in Fig. 10 as a function of the minimum truth particle separation at the sensor's surface for the first two layers of the pixel detector. It is defined as the fraction of clusters created by a particle that are then used on the reconstructed track of said particle. With the closest truth particle separated by $400 \mu \mathrm{m}$ at the IBL, the cluster assignment efficiency at this layer is in excess of $99 \%$ for the $\rho$ and three-prong $\tau$ samples, and $98 \%$ for the $B^{0}$ samples. When going to smaller separations, individual clusters start to merge and eventually only a single merged cluster remains. Since in the simpler topology $\rho \rightarrow \pi^{+} \pi^{-}$the cluster has to be assigned to a maximum of two tracks, the cluster assignment efficiency is $99 \%$ down to the smallest distances shown. In case of the $B^{0}$ and three-prong $\tau$ decays, several daughter particles are likely to contribute to a merged cluster. The NN described in Sect. 3.4 lacks the ability to distinguish between merged clusters from more than three particles and those from exactly three particles [14]. Also, the track reconstruction algorithm limits the number of tracks using the same cluster without penalties to three. As a result, at much smaller particle separations, the cluster assignment efficiency is lim-

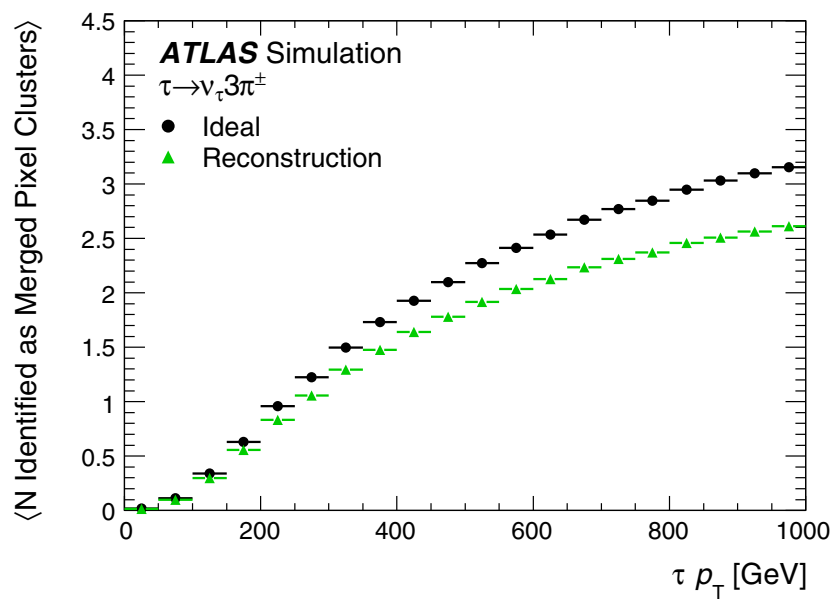

(b) $\tau^{ \pm} \rightarrow \pi^{+} \pi^{-} \pi^{ \pm} \nu_{\tau}$ sample

represents the true number of merged clusters, which would be obtained as the number of identified merged clusters in the case of perfect performance. It is required that the stable charged particles are created before the IBL 


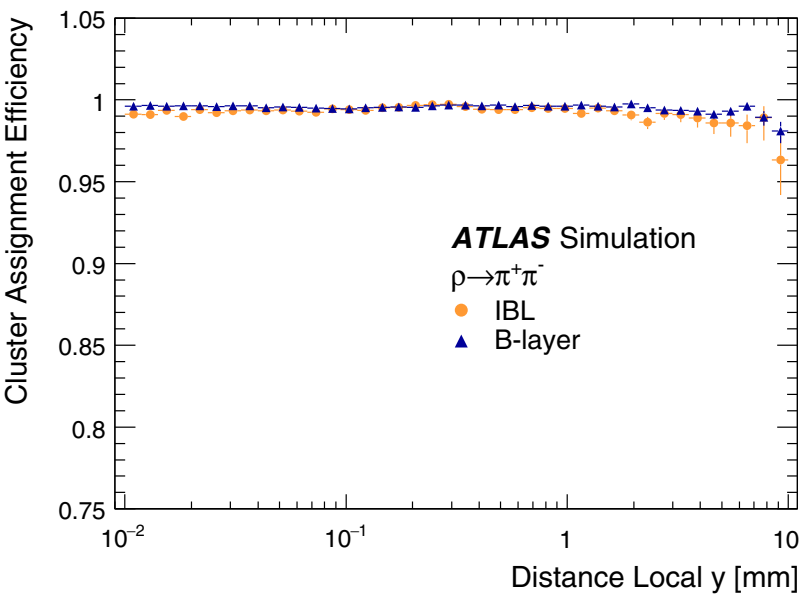

(a) $\rho \rightarrow \pi^{+} \pi^{-}$sample

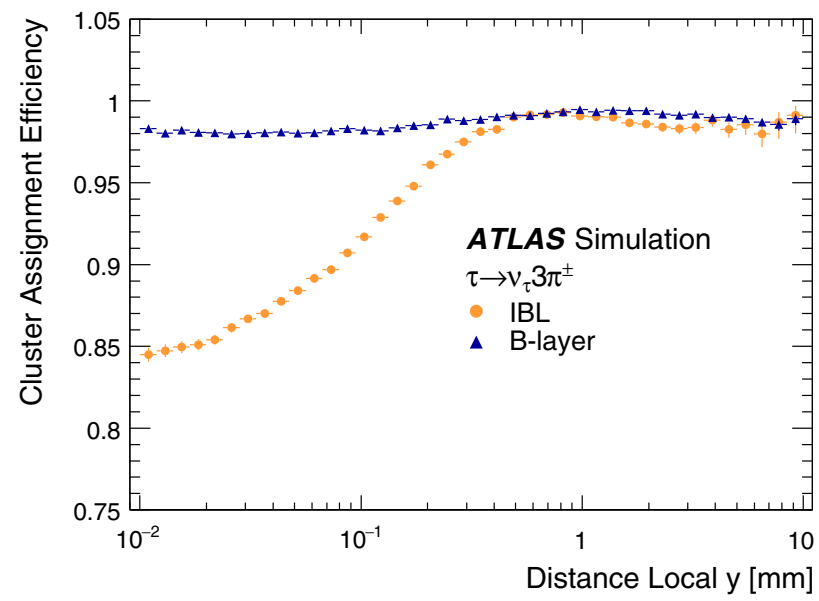

(c) $\tau \rightarrow \pi^{+} \pi^{-} \pi^{ \pm} \nu_{\tau}$ sample

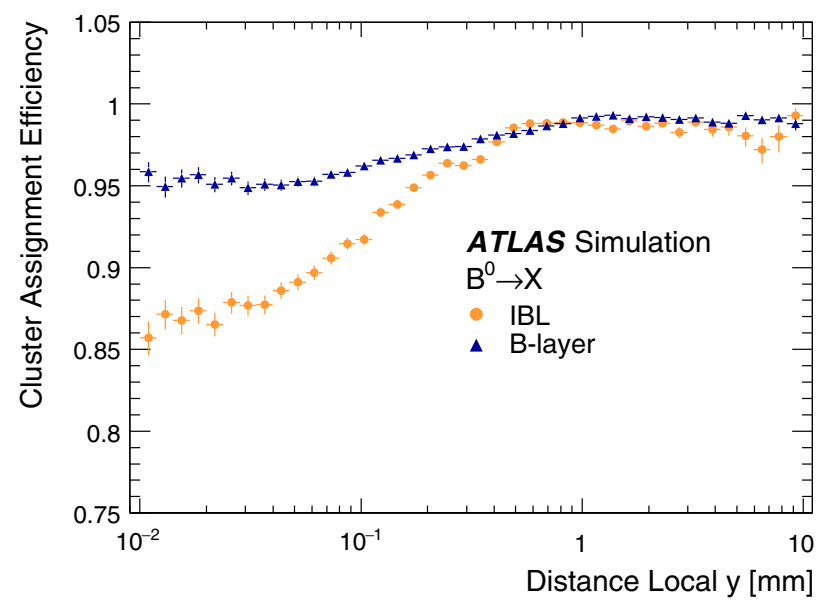

(e) $B^{0} \rightarrow X$ sample

Fig. 10 For the $\rho$ (top), three-prong $\tau$ (middle), and $B^{0}$ (bottom) samples, the efficiency with which reconstructed clusters are properly assigned to a track is shown for the two innermost pixel layers (IBL and B-layer) as a function of the minimum truth-particle separation in

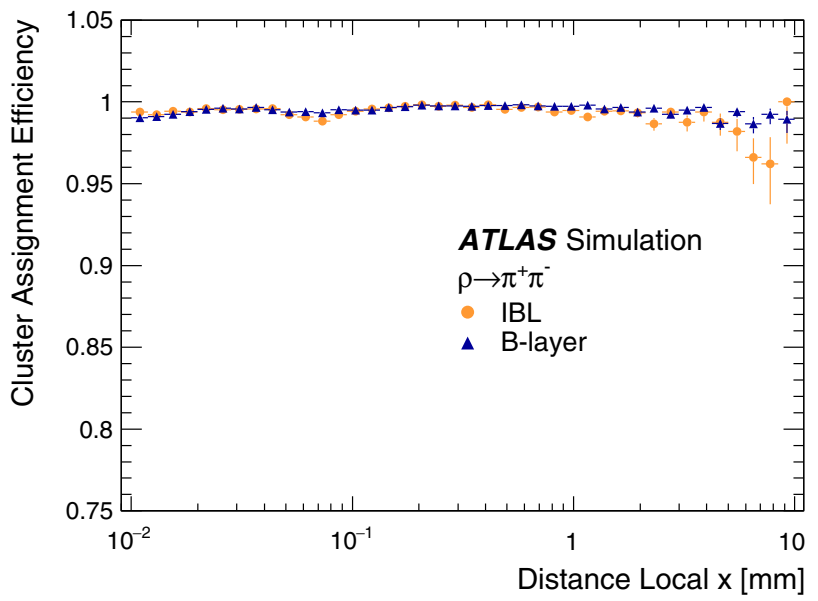

(b) $\rho \rightarrow \pi^{+} \pi^{-}$sample

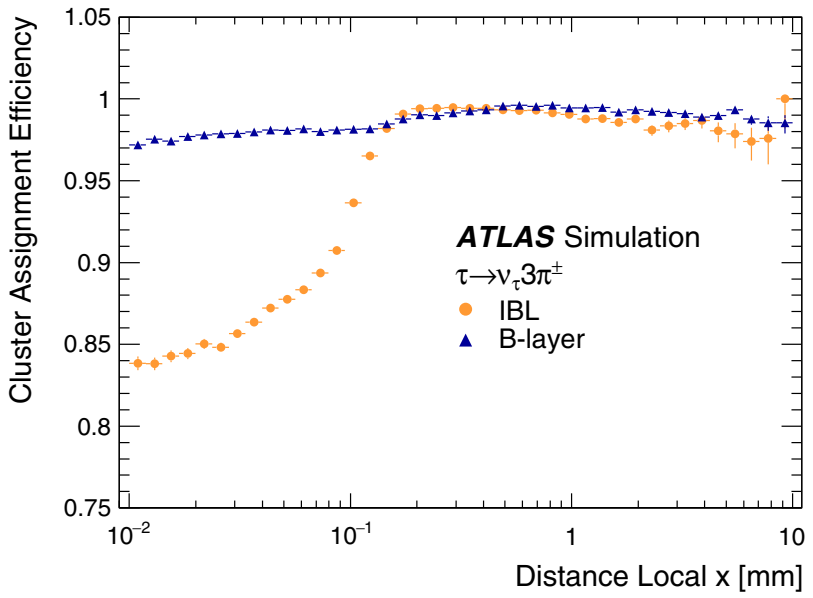

(d) $\tau \rightarrow \pi^{+} \pi^{-} \pi^{ \pm} v_{\tau}$ sample

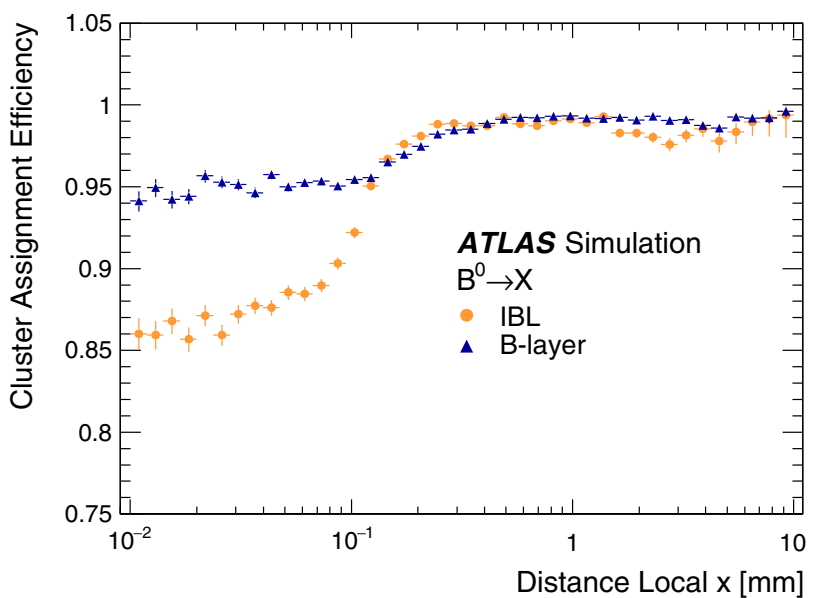

(f) $B^{0} \rightarrow X$ sample

local $y$ (left) and $x$ (right), corresponding to the pixel dimensions longitudinal and transverse to the beam axis. It is required that the stable charged particles are created before the IBL 


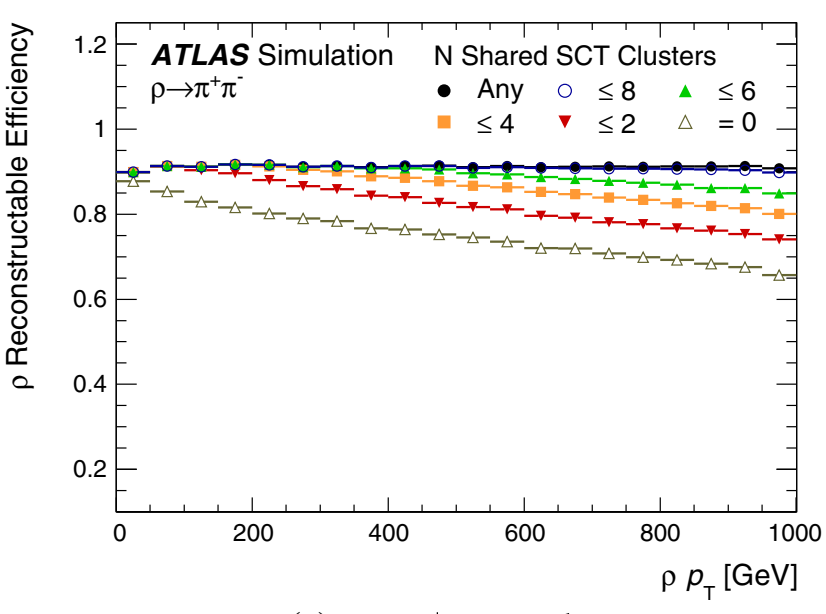

(a) $\rho \rightarrow \pi^{+} \pi^{-}$sample

Fig. 11 The reconstructable efficiency, defined as the efficiency to reconstruct all of the charged decay products of the parent particle, is shown for the $\rho$ and three-prong $\tau$ samples with various limits on the

ited in the $B^{0}$ and three-prong $\tau$ samples. The case of more than three charged particles contributing to a pixel cluster in the $B^{0}$ decay results in an additional assignment inefficiency on the B-layer.

Regardless of how well the ambiguity solver identifies merged pixel clusters and assigns them to tracks, a substantial inefficiency remains at high initial-particle momenta due to the necessary limitations on shared SCT clusters. Figure 11 shows the reconstructable efficiency of the $\rho$ and three-prong $\tau$ decays utilizing MC truth information. This is defined as the efficiency to be able to reconstruct all of the charged decay products from a given resonance having satisfied the cluster multiplicity requirements defined in Sect. 3.3. All merged pixel clusters are assumed to have been identified, so for a fixed maximum number of allowed shared SCT clusters, this represents the maximum achievable reconstruction efficiency. The loss in efficiency is exacerbated by increasing charged-particle multiplicities as in the three-prong $\tau$ sample. This limit is fixed at two shared clusters. The efficiency improvement obtained from loosening this limit is not sufficient to justify the associated increase in the proportion of fake tracks. In simulated events with several jets, the inclusive number of fake tracks increases by $25 \%$ when loosening the limit to three shared clusters.

Finally, the per-track reconstruction efficiency is shown in Fig. 12 as a function of particle $p_{\mathrm{T}}$ and production radius. The production radius is defined as the radial distance of the decay of the parent particle from the beam axis. The efficiency degrades with increased multiplicity. The visible inefficiency in all samples at low initial-particle $p_{\mathrm{T}}$ is due to inelastic interactions, such as hadronic interactions. At higher transverse momentum of the initial particle, a decrease in efficiency is driven by the increasingly collimated nature of the

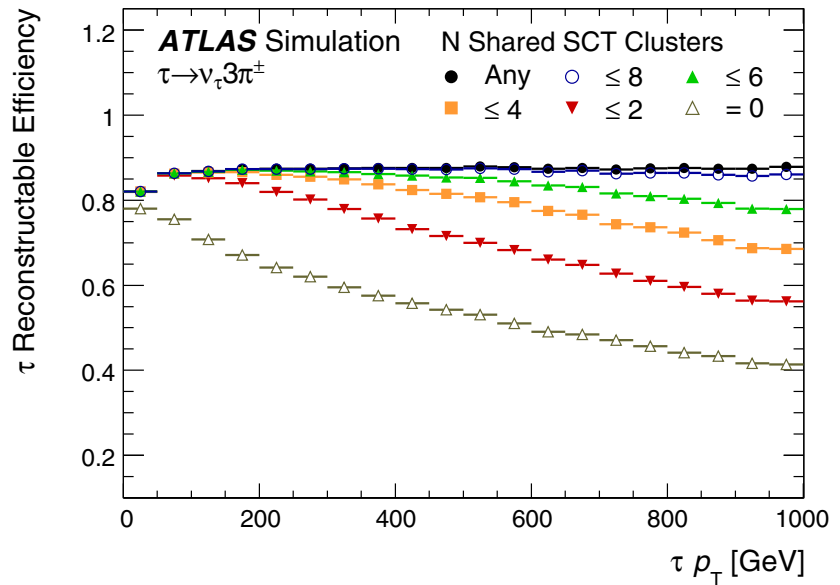

(b) $\tau^{ \pm} \rightarrow \pi^{+} \pi^{-} \pi^{ \pm} \nu_{\tau}$ sample

number of shared clusters allowed on a track candidate assuming all the merged pixel clusters have been identified as merged. It is required that the stable charged particles are created before the IBL

decay products. A decrease in efficiency is also seen with a increasing production radius as the charged particles arrive at each active layer with less average separation. The requirement on the total number of clusters for track reconstruction leads to discrete drops in efficiency at each active layer.

\subsection{Performance for tracks in jets}

In the previous sections, the performance in simple topologies is discussed. These samples are crucial for understanding the effects of charged-particle separations and multiplicities on the performance, but they are insufficient to quantify the expected performance in the dense jet environments evident in Fig. 4. As demonstrated in Sect. 5.2, samples of dijet MC events do provide a reasonable description of jets in data. The following contains studies of the track reconstruction efficiency in these samples.

Figure 13 shows the charged-primary-particle reconstruction efficiency dependence on the angular distance of a particle to the jet axis for different jet $\eta$ and $p_{\mathrm{T}}$ ranges. All charged particles studied are required to be created before the IBL. The efficiency drops rapidly towards the centre of the jet, where the charged-particle density is maximal. A slight decrease in efficiency towards the edge of the jet is consistent with an isolated-track efficiency that rises with chargedparticle $p_{\mathrm{T}}$ [27] and a decrease in the average chargedparticle $p_{\mathrm{T}}$ with distance from the jet core. The dependence of the efficiency on the jet $p_{\mathrm{T}}$ and on the production radius of the charged particle, where charged particles are not required to be created before the IBL, is shown in Fig. 14. The decrease in efficiency with production radius is from two effects. Firstly, particles created beyond the first active layers of the ID create fewer clusters. Secondly, with the shorter flight length to 


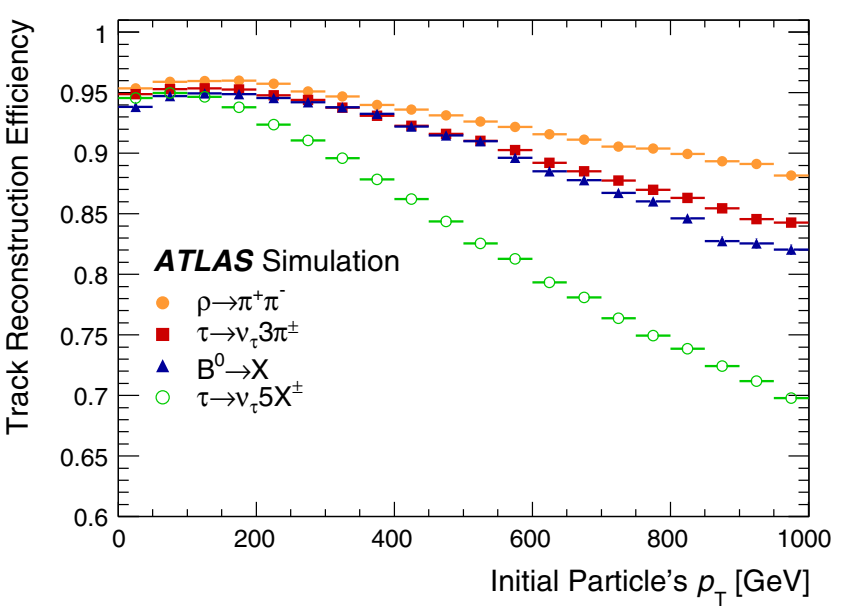

(a) Efficiency versus initial particle's $p_{\mathrm{T}}$

Fig. 12 Single-track reconstruction efficiency is shown as a a function of the initial particle's $p_{\mathrm{T}}$ when it is required that the parent particle decays before the IBL for the decay products of a $\rho$, three- and five-

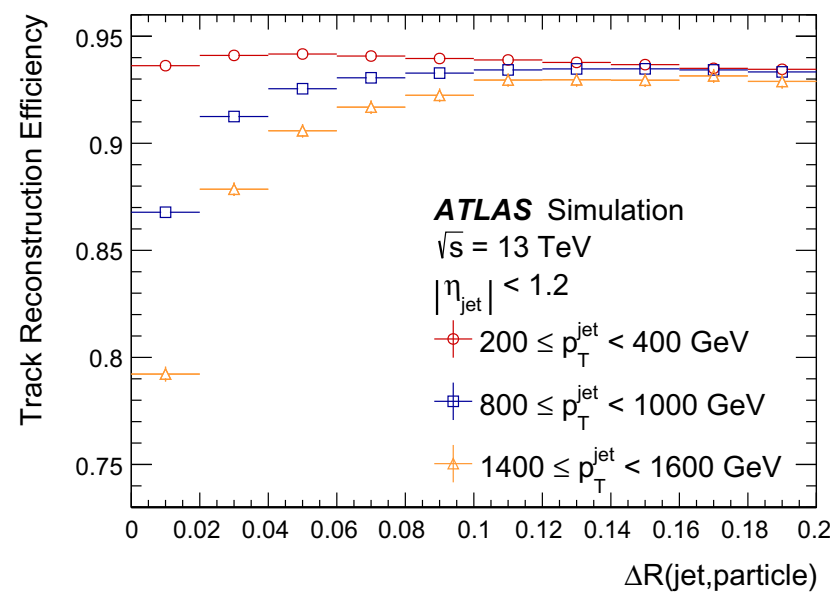

(a)

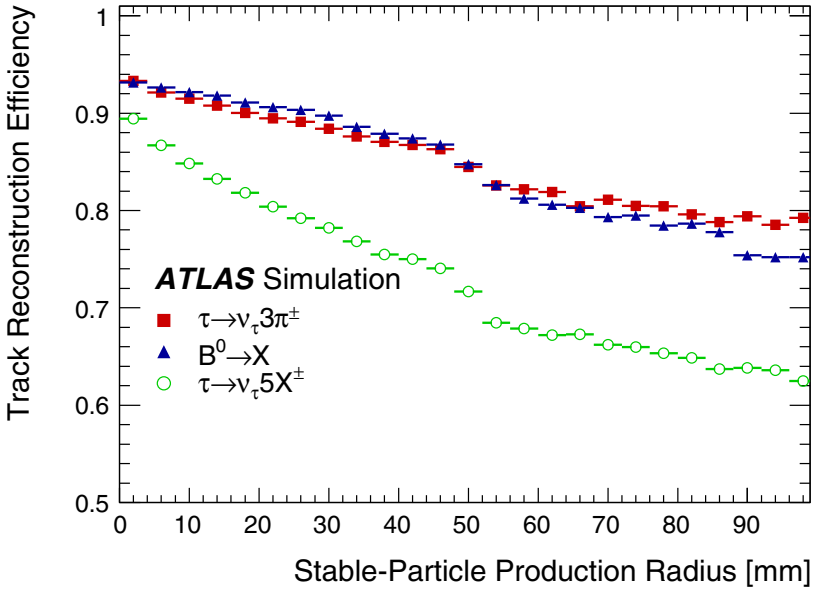

(b) Efficiency versus production radius

prong $\tau$ and a $B^{0}$ and, $\mathbf{b}$ versus the production radius for the decay products of a three- and five-prong $\tau$ as well as a $B^{0}$, where no requirement is imposed on the production radius of stable charged particles

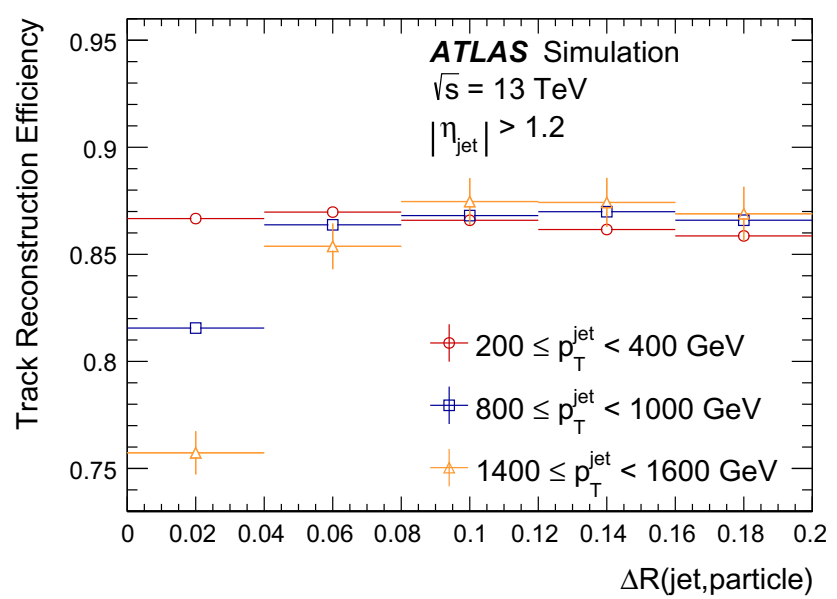

(b)

Fig. 13 The efficiency to reconstruct charged primary particles in jets with $\mathbf{a}|\eta|<1.2$ and $\mathbf{b}|\eta|>1.2$ is shown as a function of the angular distance of the particle from the jet axis for various jet $p_{\mathrm{T}}$ for simulated dijet MC events

the next active layer, the average separation between particles is smaller compared to prompt decays, producing more merged clusters. The overall trend for all efficiencies shown is the same at all $\eta$. However, the loss in absolute efficiency is exacerbated at high $|\eta|$, while the degradation at small separations between a track and the jet axis is alleviated.

\section{Measurement of track reconstruction efficiency in jets from data}

Previous sections discuss the performance of the track reconstruction in dense environments based mainly on MC simulation. This section introduces a novel method to probe this performance in data. A measurement of the fraction of tracks lost in reconstruction due to the high density and collimation of charged particles in high- $p_{\mathrm{T}}$ jets is presented for the subset of tracks with a B-layer cluster created by two charged particles.

The $\mathrm{d} E / d x$ of a charged particle traversing the pixel sensor is measured from the charge collected in the clusters associated with the reconstructed track. With single particles and thin layers, one expects the $\mathrm{d} E / d x$ measurements to approximately follow a Landau distribution [28]. A typical particle reconstructed from an LHC collision is expected to be a minimum-ionizing particle (MIP). Thus, two particles contributing to the same cluster are expected to deposit twice the energy of a single MIP. In the context of this paper, $\mathrm{d} E / d x$ is 


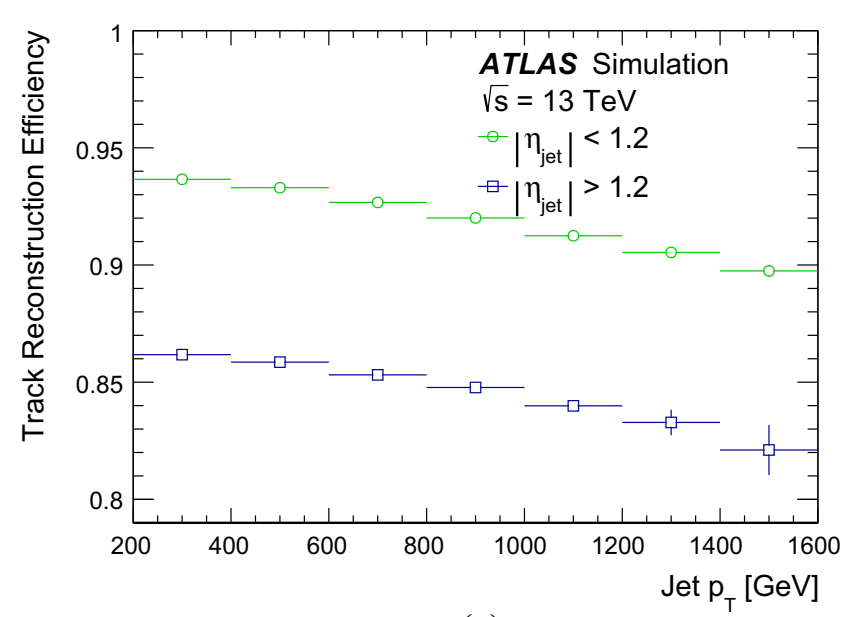

(a)

Fig. 14 The track reconstruction efficiency is compared for charged primary particles in jets with $|\eta|<1.2(|\eta|>1.2)$ for the entire jet- $p_{\mathrm{T}}$ range as a function of $\mathbf{a}$ the jet $p_{\mathrm{T}}$ and $\mathbf{b}$ the production radius of the

normalized to the material density, and it therefore has units of $\mathrm{MeVg}^{-1} \mathrm{~cm}^{2}$.

As demonstrated in the previous sections, near the jet core the charged-particle density is high and particles can be highly collimated. The tracks of these particles are thus more likely to create merged clusters, as shown in Fig. 5. By fitting the cluster $\mathrm{d} E / d x$ for reconstructed tracks near the core of the jet, single-particle clusters can be statistically separated from merged clusters. The fraction of lost tracks can therefore be inferred from the number of times only one reconstructed track is associated with a cluster $\mathrm{d} E / d x$ compatible with two MIPs. At truth-level, this fraction is defined as follows: the denominator is the number of truth particles passing the analysis selections (listed in Sect. 6.1, and including a $p_{\mathrm{T}}>10 \mathrm{GeV}$ requirement), which have a B-layer cluster created by exactly two charged particles; the numerator is the subset of these particles which failed to be reconstructed.

For the IBL, ToT is encoded in four bits. Eight bits are available in each of the remaining three pixel layers, which therefore provide an enhanced ToT resolution compared to the IBL, resulting in a superior energy resolution. For this reason, the cluster $\mathrm{d} E / d x$ values corresponding to the B-layer are used in this study.

\subsection{Track selection}

To enhance the contribution of high-quality collimated tracks and suppress fake tracks to a negligible number, additional track selections beyond those outlined in Sect. 3.3 are required for all tracks used in this analysis:

- Exactly one pixel cluster per layer,

- $p_{\mathrm{T}}>10 \mathrm{GeV}$,

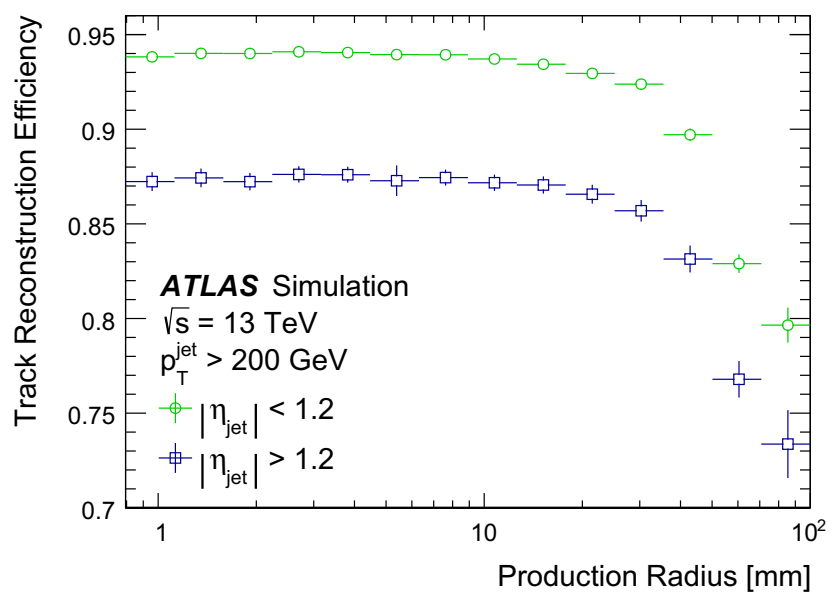

(b)

charged particle for simulated dijet MC events, where charged particles are not required to be created before the IBL

- $|\eta|<1.2$

- $\left|d_{0}^{\mathrm{BL}}\right|<1.5 \mathrm{~mm}$,

- $\left|z_{0}^{\mathrm{BL}} \sin \theta\right|<1.5 \mathrm{~mm}$,

- Minimum of six SCT clusters.

\subsection{Fit method}

A measurement distribution of cluster $\mathrm{d} E / d x$ of tracks inside the jet core is fit using two $\mathrm{d} E / d x$ template distributions: a single-track template containing mainly tracks reconstructed from a single-particle cluster, and a multiple-track template mainly made up of tracks reconstructed from a merged cluster. Both templates are derived directly from collision data or from simulation for the corresponding efficiency measurements.

As verified in simulation, most highly collimated tracks are expected to be within $\Delta R$ (jet,trk $)<0.05$ which then defines the jet core for this method. Outside the jet core, the contribution of collimated tracks is negligible, and therefore all tracks are expected to be reconstructed from a singleparticle cluster. The single-track template is created using tracks reconstructed from clusters which are neither identified as merged nor shared and that are well outside the jet core $(\Delta R($ jet,trk $)>0.1)$. The multiple-track template is taken from tracks reconstructed from either B-layer clusters identified as merged or shared B-layer clusters inside the jet core. These multiply used clusters are likely to be merged clusters.

Examples of the resulting distributions are shown in Fig. 15. The single-track template, displayed as circles in Fig. 15 , contains a single peak at the $\mathrm{d} E / d x$ value expected for a MIP traversing the B-layer of the pixel detector and a long tail to higher values compatible with a Landau distribution. Contamination of merged clusters in this template 


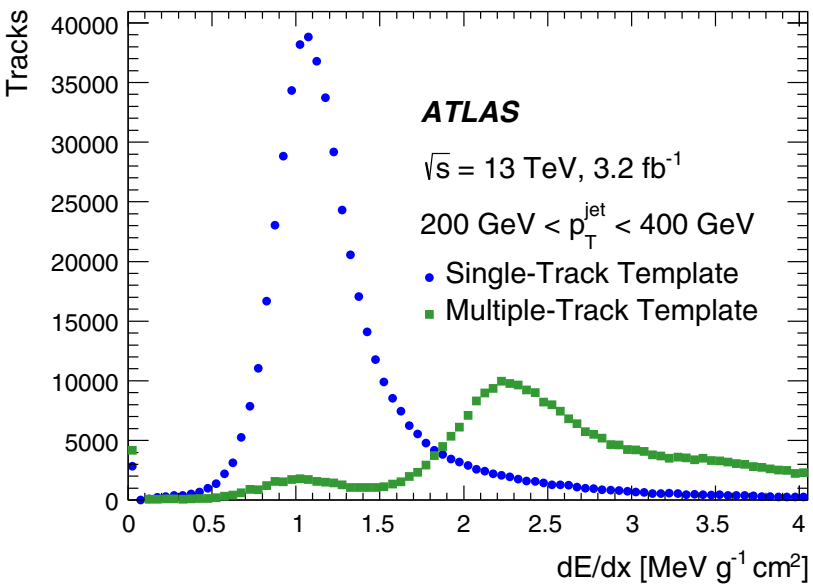

Fig. 15 Single-track and multiple-track templates for data with a jet $p_{\mathrm{T}}$ in the range $200 \mathrm{GeV}<p_{\mathrm{T}}^{\text {jet }}<400 \mathrm{GeV}$

is $0.3-0.5 \%$ in the simulation. The multiple-track template, displayed as squares in the same figure, instead exhibits a peak in the $\mathrm{d} E / d x$ range expected for two MIPs. A third, smaller peak occurs at $\mathrm{d} E / d x>3.2 \mathrm{MeVg}^{-1} \mathrm{~cm}^{2}$ for clusters created by three particles. The peak in the multiple-track template $\mathrm{d} E / d x$ distribution at values expected for one MIP is due to the fact that multiply used clusters can also originate from shared clusters or clusters identified as merged which, in truth, are not merged clusters.

The measurement distribution is created from tracks inside the jet core that are reconstructed from a cluster which is neither identified as merged nor shared. No additional requirements are made on other tracks using this cluster, including whether or not they satisfy the selections outlined in Sect. 6.1. The resulting $\mathrm{d} E / d x$ distribution contains singleparticle clusters with a peak at the energy of one MIP and a long tail to high values, as well as an enhanced contribution of merged clusters from two particles. Contributions from clusters from more than two particles are negligible. The true two-MIP clusters are created from a pair of tracks where only one track is reconstructed. Therefore, for every reconstructed track in the measurement distribution with a merged cluster, there is one particle which is not reconstructed. Using this information, the number of tracks contributing to merged Blayer clusters from two particles $\left(N_{2}^{\text {True }}\right)$ is found from the sum of the number of reconstructed particles in the multipletrack template $\left(N_{2}^{\text {Reco }}\right)$ and twice the number of lost particles $\left(N_{\text {Lost }}\right)$

$N_{2}^{\text {True }}=N_{2}^{\text {Reco }}+2 \cdot N_{\text {Lost }}$.

The sample of $\rho$ decays discussed in Sect. 5.3 is used to confirm that the multiple-track template captures merged clusters and that the second MIP peak in the measurement sample does in fact contain merged clusters where one contributing particle is not reconstructed. Therefore, to obtain the number of lost tracks $\left(N_{\text {Lost }}\right)$, the measurement distribution is fit with the two templates. The fraction of merged clusters in the measurement distribution, $F^{\text {merged }}$, is simply calculated from the post-fit number of tracks in the multipletrack template divided by the total number of tracks $\left(N_{\text {Data }}^{\text {Reco }}\right)$. Finally, the fraction of lost tracks passing through the same detector element as a reconstructed track is given by:

$$
\begin{aligned}
F^{\text {lost }_{2}} & =\frac{N_{\text {Lost }}}{N_{2}^{\text {True }}}, \\
& \approx \frac{N_{\text {Lost }}}{N_{2}^{\text {Reco }}+2 \cdot N_{\text {Lost }}},
\end{aligned}
$$

where

$N_{\text {Lost }}=F^{\text {merged }} \cdot N_{\text {Data }}^{\text {Reco }}$.

The relation is approximate due to the assumption that the lost track of a pair of tracks has the same properties (e.g. $p_{\mathrm{T}}$ and hit content) as the reconstructed track. In simulation, this assumption can be explicitly checked by requiring the truth particle corresponding to the lost track to also pass the analysis selections. This confirms that the deviation from the approximation results in a less than $1.5 \%$ change in $F^{\text {lost }_{2}}$.

To minimize the effect of clusters created by more than two particles, the fit was performed over the range 1.1-3.07 (1.26-3.2) $\mathrm{MeVg}^{-1} \mathrm{~cm}^{2}$ for data (simulation). Contributions from clusters from more than two particles in this range are of the order of a few percent. An offset in the distributions observed in MC events compared to data requires an adjustment of the respective fit ranges. The ranges are chosen to have the same fraction of clusters inside the fit range with respect to all clusters in the distribution. An imperfect description of the leading edge of the measurement distribution by the single-track template would affect the fitted result. Since the area of interest lies at much higher $\mathrm{d} E / d x$ values, the lower edge of the fit range was chosen to avoid as much as possible the leading edge of the single-particle $\mathrm{d} E / d x$ peak, while retaining a large sample for the remainder of the distribution.

To study the dependence of lost tracks on jet $p_{\mathrm{T}}$, the fit is performed in seven different bins of jet $p_{\mathrm{T}}$ ranging from $200 \mathrm{GeV}$ to $1600 \mathrm{GeV}$ in steps of $200 \mathrm{GeV}$.

The measurement is performed both on data and simulation samples. For simulation, separate templates are constructed for each jet- $p_{\mathrm{T}}$ bin. For data, the single-track and multiple-track templates are derived from the lowest jet- $p_{\mathrm{T}}$ bin, shown in Fig. 15, due to the small number of events at higher jet $p_{\mathrm{T}}$. It was verified that within the statistical uncertainty of the high- $p_{\mathrm{T}}$ bins, the templates derived from the lowest jet- $p_{\mathrm{T}}$ bin have the same shape within the fitted range. 


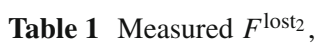
relative values of leading systematic uncertainties, and total systematic and statistical uncertainty in the fraction of lost tracks for data in bins of jet $p_{\mathrm{T}}$

\begin{tabular}{lllllll}
\hline Jet $p_{\mathrm{T}}(\mathrm{GeV})$ & $F^{\text {lost }_{2}}$ & Fit range $(\%)$ & Low- $p_{\mathrm{T}}$ temp. (\%) & Non-closure $(\%)$ & Tot. syst. (\%) & Stat. (\%) \\
\hline $200-400$ & 0.061 & 13 & 0 & 18 & 23 & 10 \\
$400-600$ & 0.063 & 12 & 7 & 11 & 17 & 6 \\
$600-800$ & 0.070 & 10 & 13 & 6 & 17 & 7 \\
$800-1000$ & 0.064 & 12 & 18 & 1 & 22 & 11 \\
$1000-1200$ & 0.067 & 12 & 21 & 0 & 24 & 15 \\
$1200-1400$ & 0.080 & 11 & 16 & 0 & 19 & 13 \\
$1400-1600$ & 0.093 & 15 & 16 & 0 & 22 & 18 \\
\hline
\end{tabular}

\subsection{Systematic uncertainties}

The resulting $F^{\text {merged }}$ exhibits a statistical uncertainty due to the finite number of entries in both the template and the measurement distributions.

Various potential sources of systematic bias were studied and are discussed below. The relative values for data are summarized in Table 1 and values for MC simulation are comparable. The measured $F^{\text {lost }_{2}}$ varies as a function of the range in $\mathrm{d} E / d x$ for which the distribution is fit. This is due to the different fractions of clusters with a $\mathrm{d} E / d x$ of two and three MIPs falling in the fitted range. The effect was estimated by increasing the fit range. The fitting process was repeated for six different ranges with the upper edge increasing in 0.2 $\mathrm{MeVg}^{-1} \mathrm{~cm}^{2}$ increments. A symmetric uncertainty, equal to the maximum change in $F^{\text {lost }_{2}}$, is applied to each jet- $p_{\mathrm{T}}$ bin. The start of the fitted range was chosen such that small variations have a negligible impact on $F^{\text {lost }_{2}}$.

A systematic uncertainty considered for data is the result of fitting all data jet- $p_{\mathrm{T}}$ bins with the templates from the lowest jet- $p_{\mathrm{T}}$ bin. This results in an overestimate of $F^{\text {lost }_{2}}$ increasing with jet $p_{\mathrm{T}}$. To account for this bias, a $p_{\mathrm{T}^{-}}$ dependent multiplicative correction was determined by comparing the $F^{\text {lost }_{2}}$ values fitted in simulation with templates from the corresponding jet- $p_{\mathrm{T}}$ bin with those obtained using a template from the lowest jet- $p_{\mathrm{T}}$ bin. This correction increases from about 10 to $25 \%$ for jets with a $p_{\mathrm{T}}$ ranging from 400 to $600 \mathrm{GeV}$ and from 1400 to $1600 \mathrm{GeV}$, respectively. This correction term was applied to data $F^{\text {lost }_{2}}$ values after completing the fitting procedure. In addition, the difference between the two simulation $F^{\text {lost }_{2}}$ values compared for the correction factor was also included as a systematic uncertainty. An additional check performed with a large simulated sample showed a 3-8\% bias in $F^{\text {lost }_{2}}$ in the studied jet- $p_{\mathrm{T}}$ range due to the fraction of tracks reconstructed from $\geq 3$ particle clusters, relative to the two-particle contribution in the multiple-track template.

To validate the method, and provide an estimate of any residual biases, a truth-based closure test was performed using simulated samples. At low jet $p_{\mathrm{T}}$, the residual $\mathrm{d} E / d x$ peak at values expected from one MIP in the multiple-track template contributes to a non-closure. Also, for all jet $p_{\mathrm{T}}$, isolated-track reconstruction efficiency, the composition of multiple-particle clusters, including particle composition and the calibration of $\mathrm{d} E / d x$ itself are all covered in this nonclosure estimate. This is already covered by the systematic uncertainty determined from changing the fit range described above, but also leads to a non-closure. In the lowest jet- $p_{\mathrm{T}}$ bin, a non-closure of approximately $+18 \%$ is observed, corresponding to an absolute overestimation of the true $F^{\text {lost }_{2}}$ of about 0.013 , but then quickly decreases with increasing jet $p_{\mathrm{T}}$. This uncertainty is included for both simulation and data with the corresponding relative values in Table 1 .

Other possible sources of uncertainty are contributions to $F^{\text {lost }_{2}}$ not originating from the density of the environment. Such contributions could come from pile-up tracks creating merged clusters with tracks in the jets, as well as lost isolated tracks. Conservative estimates based on MC studies showed that such contributions are $2-6 \%$ of the total $F^{\text {lost }_{2}}$ in the studied jet- $p_{\mathrm{T}}$ range. This effect is covered by the non-closure systematic uncertainty described above.

Uncertainties in the jet energy scale calibration and resolution have negligible impact in the analysis. Possible effects due to the binning of the $\mathrm{d} E / d x$ distributions were studied and found also to be insignificant.

\subsection{Results}

Figure 16 shows the fit result for data in two bins of jet $p_{\mathrm{T}}$. The single-track and multiple-track $\mathrm{d} E / d x$ templates provide a good description of the $\mathrm{d} E / d x$ distribution as visible from the ratio in Fig. 16.

Differences between event generators, such as different hadronization models and flavour compositions, can affect $F^{\text {lost }_{2}}$ and the overall comparison of data and MC simulation. By comparing the fit results from simulated samples made with the PYTHIA 8, SHERPA and Herwig++event generators, a generator uncertainty was derived for simulation only. For each jet- $p_{\mathrm{T}}$ bin, results from PYTHIA are taken as the central value and the largest difference of $F^{\text {lost }_{2}}$ between the three generators is symmetrized and taken as the generator uncertainty. The relative generator uncertainties in the fraction of lost tracks ranges from 4 to $37 \%$ in the different jet- $p_{\mathrm{T}}$ bins. 


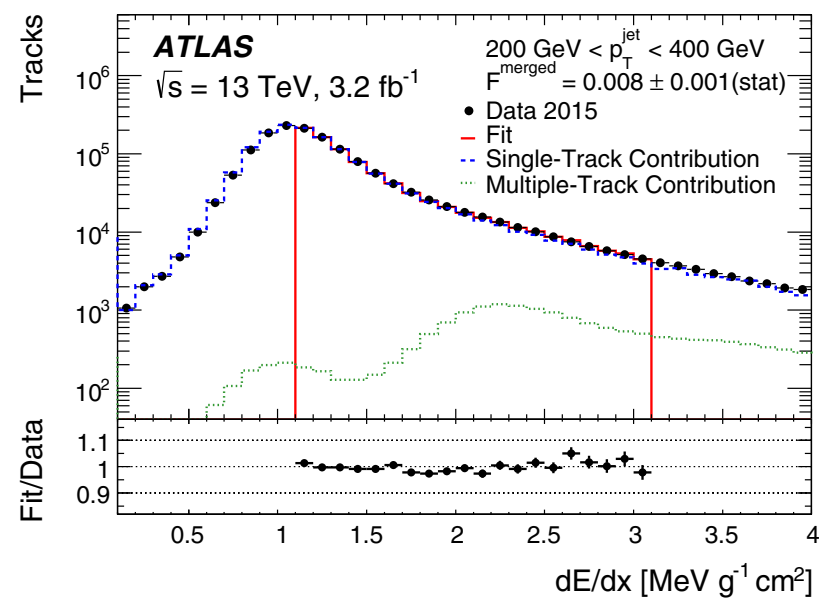

(a)

Fig. 16 Data $\mathrm{d} E / d x$ measurement distributions (black circles) with fit results (solid line) are shown for a $200 \mathrm{GeV}<p_{\mathrm{T}}^{\text {jet }}<400 \mathrm{GeV}$ and b $1000 \mathrm{GeV}<p_{\mathrm{T}}^{\text {jet }}<1200 \mathrm{GeV}$. The single-track template scaled by

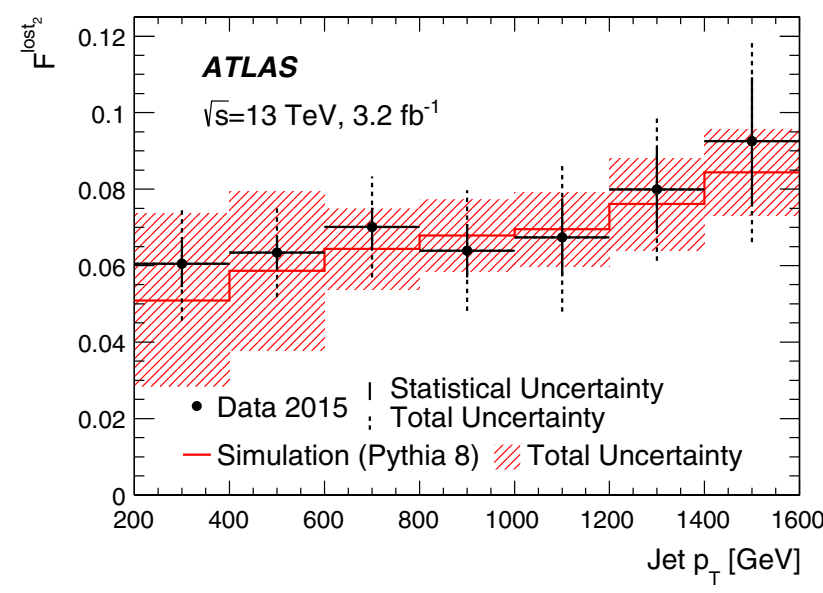

Fig. 17 The measured fraction of lost tracks, $F^{\text {lost }_{2}}$, in the jet core $(\Delta R$ (jet,trk $)<0.05)$ as a function of jet $p_{\mathrm{T}}$ for data (black circles) and simulation (red line). Vertical solid error bars indicate statistical uncertainty, while the total uncertainty is represented by dashed error bars for data and a shaded area for simulation

A comparison of $F^{\text {lost }_{2}}$ as a function of jet $p_{\mathrm{T}}$ for data and simulation is shown in Fig. 17. As the jet $p_{\mathrm{T}}$ increases, so does $F^{\text {lost }_{2}}$, with a similar trend observed in both data and simulation. This increase is caused by an increasing density of charged particles, which thereby causes higher collimation of the track pair, and is not due to confusion in correctly assigning clusters to tracks. At a certain point, the two particles are so collimated that the reconstructed tracks start to overlap completely up to the radius of the SCT detector. At that point a similar effect as shown for tracks from the $\rho$ decay in Figs. 10 and 12 occurs. The cluster assignment efficiency for reconstructed tracks remains constant with increasing jet

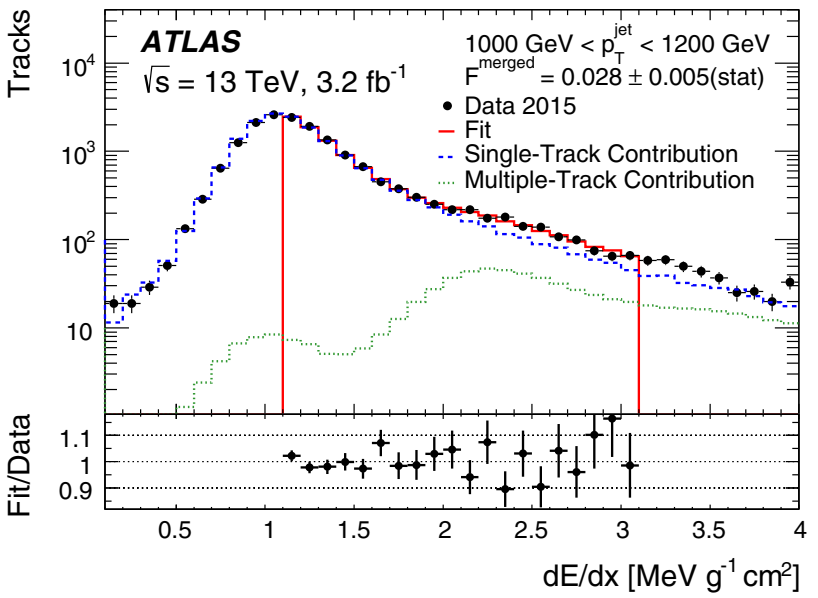

(b)

$1-F^{\text {merged }}$ is shown as the single-track contribution (dashed line) and the multiple-track template scaled by $F^{\text {merged }}$ is shown as the multipletrack contribution (dotted line). The bottom panel in each plot shows the ratio of the fit to the data within the fit range $\left(1.1-3.07 \mathrm{MeVg}^{-1} \mathrm{~cm}^{2}\right)$

$p_{\mathrm{T}}$, indicating no degradation of performance due to the environmental effects besides the second track. Only because of their increasingly collimated nature, the probability of losing one of the tracks rises. This effect was confirmed in simulation for tracks selected by this analysis.

The measurements in data and MC simulation are consistent across the whole studied jet- $p_{\mathrm{T}}$ range.

\section{Conclusion}

This paper presents the performance of the ATLAS track reconstruction chain with detailed studies in dedicated topologies, such as the cores of high- $p_{\mathrm{T}}$ jets and the decays of $\tau$-leptons, that are characterized by charged-particle separations comparable to the inner detector's sensor granularity. The ambiguity-solver stage of the reconstruction chain is described, including the usage of a neural-networkbased approach to identify pixel clusters created by multiple charged particles. The current performance is demonstrated with simulated samples of a single particle decaying to a set of collimated charged particles. In the cores of jets, the number of IBL clusters on tracks, as well as the expected track reconstruction efficiency, is robust up to the highest investigated $p_{\mathrm{T}}$ values.

A novel, fully data-driven technique, using the energy loss to identify clusters as originating from two charged particles is introduced to measure the fraction of charged particles, creating these clusters, that fail to be reconstructed. The results are presented using tracks with $p_{\mathrm{T}}$ above $10 \mathrm{GeV}$ in the core of a jet from $3.2 \mathrm{fb}^{-1}$ of $13 \mathrm{TeV}$ proton- 
proton collisions at the LHC. The measured fraction of lost tracks as a function of jet transverse momentum was found to range from $0.061 \pm 0.006$ (stat.) \pm 0.014 (syst.) to $0.093 \pm 0.017$ (stat.) \pm 0.021 (syst.) as the jet $p_{\mathrm{T}}$ increases from 200 to $1600 \mathrm{GeV}$. Data and simulation are compatible for the full studied jet- $p_{\mathrm{T}}$ range. This result can be used to minimize the uncertainty in the track reconstruction inefficiency in the cores of jets relevant for jet energy and mass calibrations as well as measurements of jet properties.

Acknowledgements We thank CERN for the very successful operation of the LHC, as well as the support staff from our institutions without whom ATLAS could not be operated efficiently. We acknowledge the support of ANPCyT, Argentina; YerPhI, Armenia; ARC, Australia; BMWFW and FWF, Austria; ANAS, Azerbaijan; SSTC, Belarus; CNPq and FAPESP, Brazil; NSERC, NRC and CFI, Canada; CERN; CONICYT, Chile; CAS, MOST and NSFC, China; COLCIENCIAS, Colombia; MSMT CR, MPO CR and VSC CR, Czech Republic; DNRF and DNSRC, Denmark; IN2P3-CNRS, CEA-DSM/IRFU, France; SRNSF, Georgia; BMBF, HGF, and MPG, Germany; GSRT, Greece; RGC, Hong Kong SAR, China; ISF, I-CORE and Benoziyo Center, Israel; INFN, Italy; MEXT and JSPS, Japan; CNRST, Morocco; NWO, Netherlands; RCN, Norway; MNiSW and NCN, Poland; FCT, Portugal; MNE/IFA, Romania; MES of Russia and NRC KI, Russian Federation; JINR; MESTD, Serbia; MSSR, Slovakia; ARRS and MIZŠ, Slovenia; DST/NRF, South Africa; MINECO, Spain; SRC and Wallenberg Foundation, Sweden; SERI, SNSF and Cantons of Bern and Geneva, Switzerland; MOST, Taiwan; TAEK, Turkey; STFC, United Kingdom; DOE and NSF, United States of America. In addition, individual groups and members have received support from BCKDF, the Canada Council, CANARIE, CRC, Compute Canada, FQRNT, and the Ontario Innovation Trust, Canada; EPLANET, ERC, ERDF, FP7, Horizon 2020 and Marie Skłodowska-Curie Actions, European Union; Investissements d'Avenir Labex and Idex, ANR, Région Auvergne and Fondation Partager le Savoir, France; DFG and AvH Foundation, Germany; Herakleitos, Thales and Aristeia programmes co-financed by EU-ESF and the Greek NSRF; BSF, GIF and Minerva, Israel; BRF, Norway; CERCA Programme Generalitat de Catalunya, Generalitat Valenciana, Spain; the Royal Society and Leverhulme Trust, United Kingdom. The crucial computing support from all WLCG partners is acknowledged gratefully, in particular from CERN, the ATLAS Tier-1 facilities at TRIUMF (Canada), NDGF (Denmark, Norway, Sweden), CC-IN2P3 (France), KIT/GridKA (Germany), INFN-CNAF (Italy), NL-T1 (Netherlands), PIC (Spain), ASGC (Taiwan), RAL (UK) and BNL (USA), the Tier-2 facilities worldwide and large non-WLCG resource providers. Major contributors of computing resources are listed in Ref. [29].

Open Access This article is distributed under the terms of the Creative Commons Attribution 4.0 International License (http://creativecomm ons.org/licenses/by/4.0/), which permits unrestricted use, distribution, and reproduction in any medium, provided you give appropriate credit to the original author(s) and the source, provide a link to the Creative Commons license, and indicate if changes were made. Funded by SCOAP ${ }^{3}$.

\section{References}

1. J.M. Butterworth, A.R. Davison, M. Rubin, G.P. Salam, Jet substructure as a new Higgs search channel at the LHC. Phys. Rev. Lett. 100, 242001 (2008). arXiv:0802.2470 [hep-ph]
2. ATLAS Collaboration, The ATLAS experiment at the CERN large Hadron collider. JINST 3, S08003 (2008)

3. ATLAS Collaboration, ATLAS insertable B-layer technical design report, ATLAS-TDR-19 (2010). https://cds.cern.ch/record/ 1291633

4. ATLAS Collaboration, ATLAS insertable B-layer technical design report addendum, ATLAS-TDR-19-ADD-1 (2012). https://cds. cern.ch/record/1451888

5. G. Aad et al., ATLAS pixel detector electronics and sensors. JINST 3, P07007 (2008)

6. ATLAS TRT Collaboration, E. Abat et al., The ATLAS TRT barrel detector. JINST 3, P02014 (2008)

7. D. Wicke, A new algorithm for solving tracking ambiguities, LC-TOOL-1999-007-TESLA 219 (1999). flc.desy.de/lcnotes/notes/LC-TOOL-1999-007-TESLA.ps.gz

8. T. Cornelissen et al., The new ATLAS track reconstruction (NEWT). J. Phys. Conf. Ser. 119, 032014 (2008)

9. A. Rosenfeld, J. Faltz, Sequential operations in digital picture processing. J. ACM 13, 471-494 (1966)

10. E. Belau et al., Charge collection in silicon strip detectors. Nucl. Instrum. Meth. 214, 253 (1983)

11. R. Frühwirth, Application of Kalman filtering to track and vertex fitting. Nucl. Instrum. Methods A 262, 444 (1987)

12. ATLAS Collaboration, Muon reconstruction performance of the ATLAS detector in proton-proton collision data at $\sqrt{s}=13 \mathrm{TeV}$. Eur. Phys. J. C 76, 292 (2016). arXiv:1603.05598 [hep-ex]

13. ATLAS Collaboration, Reconstruction of primary vertices at the ATLAS experiment in Run 1 proton-proton collisions at the LHC. Eur. Phys. J. C. (Submitted to). arXiv:1611.10235 [physics.ins-det]

14. ATLAS Collaboration, A neural network clustering algorithm for the ATLAS silicon pixel detector. JINST 9, P09009 (2014). arXiv: 1406.7690 [hep-ex]

15. T. Sjöstrand, S. Mrenna, P.Z. Skands, A brief introduction to PYTHIA 8.1. Comput. Phys. Commun. 178, 852 (2008). arXiv:0710.3820 [hep-ph]

16. ATLAS Collaboration, ATLAS Run 1 Pythia8 tunes, ATL-PHYSPUB-2014-021 (2014). https://cds.cern.ch/record/1966419

17. NNPDF Collaboration, R.D. Ball et al., Parton distributions with LHC data. Nucl. Phys. B 867, 244 (2013). arXiv:1207.1303 [hep$\mathrm{ph}]$

18. M. Bahr et al., Herwig++ physics and manual. Eur. Phys. J. C 58, 639 (2008). arXiv:0803.0883 [hep-ph]

19. T. Gleisberg et al., Event generation with SHERPA 1.1. JHEP 02, 007 (2009). arXiv:0811.4622 [hep-ph]

20. J. Pumplin et al., New generation of parton distributions with uncertainties from global QCD analysis. JHEP 07, 012 (2002). arXiv:hep-ph/0201195 [hep-ph]

21. H.-L. Lai et al., New parton distributions for collider physics. Phys. Rev. D 82, 074024 (2010). arXiv:1007.2241 [hep-ph]

22. ATLAS Collaboration, The ATLAS simulation infrastructure. Eur. Phys. J. C 70, 823 (2010). arXiv:1005.4568 [hep-ex]

23. S. Agostinelli et al., GEANT4: a simulation toolkit. Nucl. Instrum. Methods A 506, 250 (2003)

24. ATLAS Collaboration, Topological cell clustering in the ATLAS calorimeters and its performance in LHC Run 1. Eur. Phys. J. C. (Submitted to). arXiv:1603.02934 [hep-ex]

25. M. Cacciari, G.P. Salam, G. Soyez, The anti- $k_{t}$ jet clustering algorithm. JHEP 04, 063 (2008). arXiv:0802.1189 [hep-ph]

26. ATLAS Collaboration, Jet energy scale measurements and their systematic uncertainties in proton-proton collisions at $\operatorname{sqrt}(\mathrm{s})=$ $13 \mathrm{TeV}$ with the ATLAS detector. Phys. Rev. D. (Submitted to). arXiv:1703.09665 [hep-ex]

27. ATLAS Collaboration, Charged-particle distributions at low transverse momentum in $\sqrt{s}=13 \mathrm{TeV} p p$ interactions measured with the ATLAS detector at the LHC. Eur. Phys. J. C 76, 502 (2016). arXiv:1606.01133 [hep-ex] 
28. Particle Data Group, K.A. Olive et al, Review of particle physics. Chin. Phys. C 38, 090001 (2014)
29. ATLAS Collaboration, ATLAS Computing Acknowledgements 2016-2017, ATL-GEN-PUB-2016-002. https://cds.cern.ch/ record/2202407

\section{ATLAS Collaboration}

M. Aaboud ${ }^{137 d}$, G. Aad ${ }^{88}$, B. Abbott ${ }^{115}$, J. Abdallah ${ }^{8}$, O. Abdinov ${ }^{12, *}$, B. Abeloos ${ }^{119}$, S. H. Abidi ${ }^{161}$, O. S. AbouZeid ${ }^{139}$, N. L. Abraham ${ }^{151}$, H. Abramowicz ${ }^{155}, \quad$ H. Abreu ${ }^{154}$, R. Abreu ${ }^{118}$, Y. Abulaiti ${ }^{148 a, 148 b}$, B. S. Acharya ${ }^{167 a, 167 b, a}$, S. Adachi ${ }^{157}$, L. Adamczyk ${ }^{41 a}$, J. Adelman ${ }^{110}$, M. Adersberger ${ }^{102}$, T. Adye ${ }^{133}$, A. A. Affolder ${ }^{139}$, T. Agatonovic-Jovin ${ }^{14}$, C. Agheorghiesei ${ }^{28 c}$, J. A. Aguilar-Saavedra ${ }^{128 a, 128 f}$, S. P. Ahlen ${ }^{24}$, F. Ahmadov ${ }^{68 b}$, G. Aielli ${ }^{135 a, 135 b}$, S. Akatsuka ${ }^{71}$,

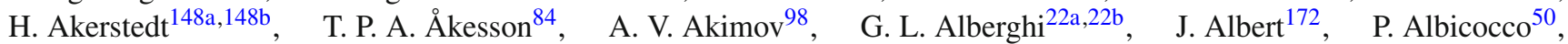

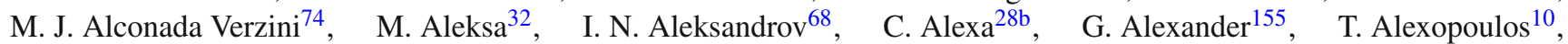

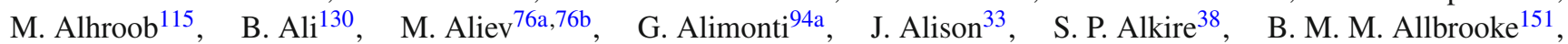
B. W. Allen ${ }^{118}$, P. P. Allport ${ }^{19}$, A. Aloisio ${ }^{106 a, 106 b}, \quad$ A. Alonso ${ }^{39}$, F. Alonso ${ }^{74}$, C. Alpigiani ${ }^{140}$, A. A. Alshehri ${ }^{56}$,

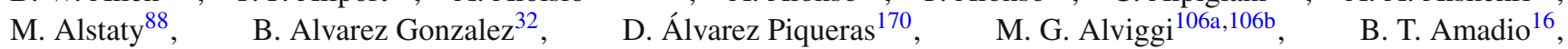
Y. Amaral Coutinho ${ }^{26 a}$, C. Amelung ${ }^{25}$, D. Amidei ${ }^{92}$, S. P. Amor Dos Santos ${ }^{128 a, 128 c}$, A. Amorim ${ }^{128 a, 128 b}$, S. Amoroso ${ }^{32}$,

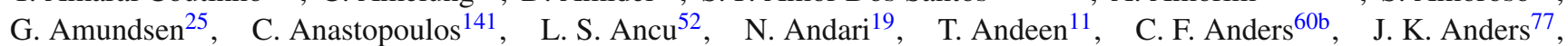
K. J. Anderson ${ }^{33}$, A. Andreazza ${ }^{94 a}$,94b, V. Andrei ${ }^{60 a}$, S. Angelidakis ${ }^{9}$, I. Angelozzi ${ }^{109}$, A. Angerami ${ }^{38}$, A. V. Anisenkov ${ }^{111 c}$,

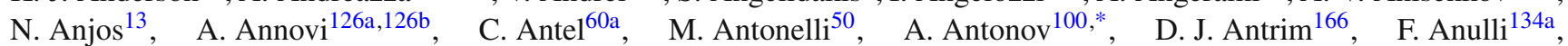
M. Aoki69, L. Aperio Bella ${ }^{32}$, G. Arabidze ${ }^{93}, \quad$ Y. Arai69, J. P. Araque ${ }^{128 a}$, V. Araujo Ferraz ${ }^{26 a}$, A. T. H. Arce ${ }^{48}$, R. E. Ardell ${ }^{80}$, F. A. Arduh ${ }^{74}$, J.-F. Arguin ${ }^{97}$, S. Argyropoulos ${ }^{66}$, M. Arik ${ }^{20 a}$, A. J. Armbruster ${ }^{145}$, L. J. Armitage ${ }^{79}$, O. Arnaez ${ }^{161}$, H. Arnold ${ }^{51}$, M. Arratia ${ }^{30}$, O. Arslan' ${ }^{23}$, A. Artamonov ${ }^{99}$, G. Artoni ${ }^{122}$, S. Artz ${ }^{86}$, S. Asai ${ }^{157}$, N. Asbah ${ }^{45}$, A. Ashkenazi ${ }^{155}$, L. Asquith ${ }^{151}$, K. Assamagan ${ }^{27}$, R. Astalos ${ }^{146 a}$, M. Atkinson ${ }^{169}$, N. B. Atlay ${ }^{143}$, K. Augsten ${ }^{130}$, G. Avolio $^{32}$, B. Axen $^{16}$, M. K. Ayoub ${ }^{119}$, G. Azuelos ${ }^{97 d}$, A. E. Baas ${ }^{60 a}$, M. J. Baca ${ }^{19}$, H. Bachacou ${ }^{138}$, K. Bachas ${ }^{76 a, 76 b}$, M. Backes ${ }^{122}$, M. Backhaus ${ }^{32}$, P. Bagnaia ${ }^{134 a, 134 b}$, H. Bahrasemani ${ }^{144}$, J. T. Baines ${ }^{133}$, M. Bajic ${ }^{39}$, O. K. Baker ${ }^{179}$, E. M. Baldin ${ }^{111}$, P. Balek ${ }^{175}$, F. Balli ${ }^{138}$, W. K. Balunas ${ }^{124}$, E. Banas ${ }^{42}$, Sw. Banerjee ${ }^{176 e}$, A. A. E. Bannoura ${ }^{178}$, L. Barak $^{32}$, E. L. Barberio ${ }^{91}$, D. Barberis ${ }^{53 a, 53 b}$, M. Barbero ${ }^{88}$, T. Barillari ${ }^{103}$, M.-S. Barisits ${ }^{32}$, T. Barklow ${ }^{145}$, N. Barlow ${ }^{30}$, S. L. Barnes ${ }^{36 c}$, B. M. Barnett ${ }^{133}$, R. M. Barnett ${ }^{16}$, Z. Barnovska-Blenessy ${ }^{36 a}$, A. Baroncelli ${ }^{136 a}$, G. Barone ${ }^{25}$, A. J. Barr ${ }^{122}$, L. Barranco Navarro ${ }^{170}$, F. Barreiro ${ }^{85}$, J. Barreiro Guimarães da Costa ${ }^{35 a}$, R. Bartoldus ${ }^{145}$, A. E. Barton ${ }^{75}$, P. Bartos ${ }^{146 a}$, A. Basalaev ${ }^{125}$, A. Bassalat ${ }^{119 f}$, R. L. Bates ${ }^{56}$, S. J. Batista ${ }^{161}$, J. R. Batley ${ }^{30}$, M. Battaglia ${ }^{139}$, M. Bauce ${ }^{134 a, 134 b}$, F. Bauer ${ }^{138}$, H. S. Bawa ${ }^{145 g}$, J. B. Beacham ${ }^{113}$, M. D. Beattie ${ }^{75}$, T. Beau ${ }^{83}$, P. H. Beauchemin ${ }^{165}$, P. Bechtle ${ }^{23}$, H. P. Beck ${ }^{18 h}$, K. Becker ${ }^{122}$, M. Becker ${ }^{86}$, M. Beckingham ${ }^{173}$, C. Becot ${ }^{112}$, A. J. Beddall ${ }^{20 d}$, A. Beddall ${ }^{20 b}$, V. A. Bednyakov ${ }^{68}$, M. Bedognetti ${ }^{109}$, C. P. Bee ${ }^{150}$, T. A. Beermann ${ }^{32}$, M. Begalli26a, M. Begel ${ }^{27}$, J. K. Behr ${ }^{45}$, A. S. Bell ${ }^{81}$, G. Bella ${ }^{155}$, L. Bellagamba ${ }^{22 a}$, A. Bellerive ${ }^{31}$, M. Bellomo ${ }^{154}$, K. Belotskiy ${ }^{100}$, O. Beltramello ${ }^{32}$, N. L. Belyaev ${ }^{100}$, O. Benary ${ }^{155, *}$, D. Benchekroun ${ }^{137 a}$, M. Bender ${ }^{102}$, K. Bendtz ${ }^{148 a, 148 b}$, N. Benekos ${ }^{10}$, Y. Benhammou ${ }^{155}$, E. Benhar Noccioli ${ }^{179}$, J. Benitez ${ }^{66}$, D. P. Benjamin ${ }^{48}$, M. Benoit ${ }^{52}$, J. R. Bensinger ${ }^{25}$, S. Bentvelsen ${ }^{109}$, L. Beresford ${ }^{122}$, M. Beretta ${ }^{50}$, D. Berge ${ }^{109}$, E. Bergeaas Kuutmann ${ }^{168}$, N. Berger ${ }^{5}$, J. Beringer ${ }^{16}$, S. Berlendis ${ }^{58}$, N. R. Bernard ${ }^{89}$, G. Bernardi ${ }^{83}$, C. Bernius $^{145}$, F. U. Bernlochner ${ }^{23}$, T. Berry ${ }^{80}$, P. Berta ${ }^{131}$, C. Bertella ${ }^{35 a}$, G. Bertoli ${ }^{148 a}$, 148b , F. Bertolucci ${ }^{126 a, 126 b}$, I. A. Bertram ${ }^{75}$, C. Bertsche ${ }^{45}$, D. Bertsche ${ }^{115}$, G. J. Besjes ${ }^{39}$, O. Bessidskaia Bylund ${ }^{148 a, 148 b}$, M. Bessner ${ }^{45}$, N. Besson ${ }^{138}$, C. Betancourt ${ }^{51}$, A. Bethani ${ }^{87}$, S. Bethke ${ }^{103}$, A. J. Bevan ${ }^{79}$, J. Beyer ${ }^{103}$, R. M. Bianchi ${ }^{127}$, O. Biebel ${ }^{102}$, D. Biedermann ${ }^{17}$, R. Bielski ${ }^{87}$, N. V. Biesuz ${ }^{126 a, 126 b}$, M. Biglietti ${ }^{136 a}, \quad$ T. R. V. Billoud ${ }^{97}, \quad$ H. Bilokon ${ }^{50}, \quad$ M. Bindi ${ }^{57}, \quad$ A. Bingul ${ }^{20 b}$,

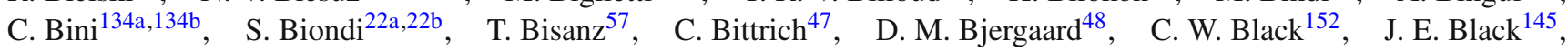
K. M. Black ${ }^{24}$, R. E. Blair ${ }^{6}$, T. Blazek ${ }^{146, a}$, I. Bloch ${ }^{45}$, C. Blocker ${ }^{25}$, A. Blue ${ }^{56}$, W. Blum ${ }^{86, *}$, U. Blumenschein ${ }^{79}$, S. Blunier ${ }^{34 a}$, G. J. Bobbink ${ }^{109}$, V. S. Bobrovnikov ${ }^{111 c}$, S. S. Bocchetta ${ }^{84}$, A. Bocci ${ }^{48}$, C. Bock ${ }^{102}$, M. Boehler ${ }^{51}$,

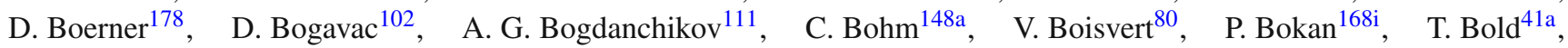
A. S. Boldyrev ${ }^{101}$, A. E. Bolz ${ }^{60 b}$, M. Bomben ${ }^{83}, \quad$ M. Bona ${ }^{79}, \quad$ M. Boonekamp ${ }^{138}$, A. Borisov ${ }^{132}$, G. Borissov ${ }^{75}$, J. Bortfeldt ${ }^{32}$, D. Bortoletto ${ }^{122}$, V. Bortolotto ${ }^{62 \mathrm{a}, 62 \mathrm{~b}, 62 \mathrm{c}}$, D. Boscherini $^{22 \mathrm{a}}$, M. Bosman $^{13}$, J. D. Bossio Sola ${ }^{29}$, J. Boudreau $^{127}$, J. Bouffard ${ }^{2}$, E. V. Bouhova-Thacker ${ }^{75}$, D. Boumediene ${ }^{37}$, C. Bourdarios ${ }^{119}$, S. K. Boutle ${ }^{56}$, A. Boveia ${ }^{113}$, J. Boyd ${ }^{32}$, I. R. Boyko ${ }^{68}$, J. Bracinik ${ }^{19}$ ， A. Brandt ${ }^{8}$ ， G. Brandt ${ }^{57}$ ， O. Brandt ${ }^{60 a}$ ， U. Bratzler ${ }^{158}$ ， B. Brau ${ }^{89}$, J. E. Brau ${ }^{118}$, W. D. Breaden Madden ${ }^{56}$, K. Brendlinger ${ }^{45}$, A. J. Brennan ${ }^{91}$, L. Brenner ${ }^{109}$, R. Brenner ${ }^{168}$, S. Bressler ${ }^{175}$, D. L. Briglin ${ }^{19}$, T. M. Bristow ${ }^{49}$, D. Britton ${ }^{56}$, D. Britzger ${ }^{45}$, F. M. Brochu ${ }^{30}$, I. Brock ${ }^{23}$, R. Brock ${ }^{93}$, G. Brooijmans ${ }^{38}$, T. Brooks ${ }^{80}$, W. K. Brooks ${ }^{34 \mathrm{~b}}$, J. Brosamer ${ }^{16}$, E. Brost ${ }^{110}$, J. H Broughton ${ }^{19}$, P. A. Bruckman de Renstrom ${ }^{42}$, D. Bruncko ${ }^{146 b}$, 
A. Bruni ${ }^{22 a}$ ， G. Bruni $22 \mathrm{a}$ ， L. S. Bruni ${ }^{109}$ ，BH Brunt ${ }^{30}$, M. Bruschi ${ }^{22 a}$ ， N. Bruscino ${ }^{23}$ ， P. Bryant ${ }^{33}$, L. Bryngemark ${ }^{45}$, T. Buanes ${ }^{15}$, Q. Buat ${ }^{144}$, P. Buchholz ${ }^{143}$, A. G. Buckley ${ }^{56}$, I. A. Budagov ${ }^{68}$, F. Buehrer ${ }^{51}$, M. K. Bugge ${ }^{121}$, O. Bulekov ${ }^{100}$, D. Bullock ${ }^{8}$, T. J. Burch ${ }^{110}$, H. Burckhart ${ }^{32}$, S. Burdin ${ }^{77}$, C. D. Burgard ${ }^{51}$, A. M. Burger ${ }^{5}$, B. Burghgrave ${ }^{110}$, K. Burka ${ }^{42}$, S. Burke ${ }^{133}$, I. Burmeister ${ }^{46}$, J. T. P. Burr ${ }^{122}$, E. Busato ${ }^{37}$, D. Büscher ${ }^{51}$, V. Büscher ${ }^{86}$, P. Bussey ${ }^{56}$, J. M. Butler ${ }^{24}$, C. M. Buttar ${ }^{56}$, J. M. Butterworth ${ }^{81}$, P. Butti ${ }^{32}$, W. Buttinger ${ }^{27}$, A. Buzatu ${ }^{35 c}$, A. R. Buzykaev ${ }^{111 c}$, S. Cabrera Urbán ${ }^{170}$,

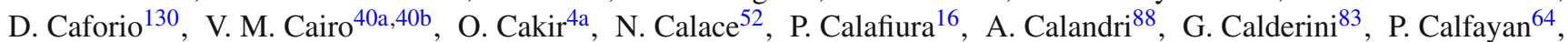
G. Callea ${ }^{40 a, 40 b}$, L. P. Caloba ${ }^{26 a}$, S. Calvente Lopez $^{85}$, D. Calvet ${ }^{37}$, S. Calvet ${ }^{37}$, T. P. Calvet ${ }^{88}$, R. Camacho Toro ${ }^{33}$, S. Camarda ${ }^{32}$, P. Camarri ${ }^{135 a, 135 b}$, D. Cameron ${ }^{121}$, R. Caminal Armadans ${ }^{169}, \quad$ C. Camincher ${ }^{58}, \quad$ S. Campana ${ }^{32}$, M. Campanelli ${ }^{81}$, A. Camplani ${ }^{94 a, 94 b}$, A. Campoverde ${ }^{143}$, V. Canale ${ }^{106 a, 106 b}$, M. Cano Bret ${ }^{36 c}$, J. Cantero ${ }^{116}$, T. Cao ${ }^{155}$, M. D. M. Capeans Garrido ${ }^{32}$, I. Caprini ${ }^{28 b}, \quad$ M. Caprini ${ }^{28 b}, \quad$ M. Capua ${ }^{40 a, 40 b}, \quad$ R. M. Carbone ${ }^{38}, \quad$ R. Cardarelli ${ }^{135 a}$, F. Cardillo ${ }^{51}$, I. Carli ${ }^{131}$, T. Carli $^{32}$, G. Carlino ${ }^{106 a}$, B. T. Carlson ${ }^{127}$, L. Carminati ${ }^{94 a, 94 b}$, R. M. D. Carney ${ }^{148 a, 148 b}$, S. Caron ${ }^{108}$, E. Carquin ${ }^{34 b}$, S. Carrá94a,94b , G. D. Carrillo-Montoya ${ }^{32}$, J. Carvalho ${ }^{128 a}, 128 c$, D. Casadei ${ }^{19}$, M. P. Casado ${ }^{13 j}$,

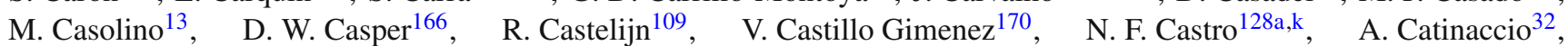
J. R. Catmore ${ }^{121}$, A. Cattai ${ }^{32}$, J. Caudron ${ }^{23}$, V. Cavaliere ${ }^{169}$, E. Cavallaro ${ }^{13}$, D. Cavalli ${ }^{94 a}$, M. Cavalli-Sforza ${ }^{13}$,

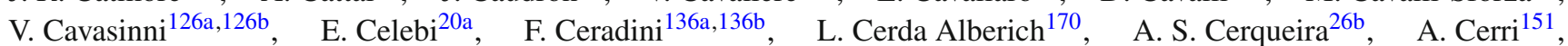
L. Cerrito ${ }^{135 a, 135 b}$, F. Cerutti ${ }^{16}, \quad$ A. Cervelli ${ }^{18}, \quad$ S. A. Cetin ${ }^{20 c}$, A. Chafaq ${ }^{137 a}, \quad$ D. Chakraborty ${ }^{110}$, S. K. Chan ${ }^{59}$, W. S. Chan ${ }^{109}$, Y. L. Chan ${ }^{62 a}$, P. Chang ${ }^{169}$, J. D. Chapman ${ }^{30}$, D. G. Charlton ${ }^{19}$, C. C. Chau ${ }^{161}$, C. A. Chavez Barajas ${ }^{151}$, S. Che ${ }^{113}$, S. Cheatham ${ }^{167 a, 167 c}$, A. Chegwidden ${ }^{93}$, S. Chekanov ${ }^{6}, \quad$ S. V. Chekulaev ${ }^{163 a}$, G. A. Chelkov ${ }^{681}$, M. A. Chelstowska ${ }^{32}$, C. Chen ${ }^{67}$, H. Chen ${ }^{27}, \quad$ S. Chen ${ }^{35 b}, \quad$ S. Chen ${ }^{157}, \quad$ X. Chen ${ }^{35 c, m}, \quad$ Y. Chen ${ }^{70}, \quad$ H. C. Cheng ${ }^{92}$, H. J. Cheng ${ }^{35 a}$, A. Cheplakov ${ }^{68}$, E. Cheremushkina ${ }^{132}$, R. Cherkaoui El Moursli ${ }^{137 e}$, V. Chernyatin ${ }^{27, *}$ E. Cheu', L. Chevalier ${ }^{138}$, V. Chiarella ${ }^{50}$, G. Chiarelli ${ }^{126 a, 126 b}$, G. Chiodini ${ }^{76 a}$, A. S. Chisholm ${ }^{32}$, A. Chitan ${ }^{28 b}$, Y. H. Chiu ${ }^{172}$, M. V. Chizhov ${ }^{68}$, K. Choi ${ }^{64}$, A. R. Chomont ${ }^{37}$, S. Chouridou ${ }^{156}$, V. Christodoulou ${ }^{81,}$ D. Chromek-Burckhart ${ }^{32}$,

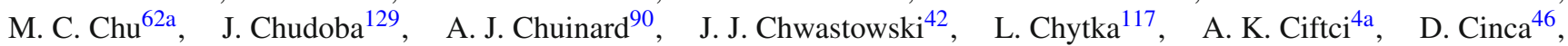
V. Cindro ${ }^{78}$, I. A. Cioara ${ }^{23}$, C. Ciocca ${ }^{22 a, 22 b}, \quad$ A. Ciocio ${ }^{16}, \quad$ F. Cirotto ${ }^{106 a, 106 b}, \quad$ Z. H. Citron ${ }^{175}, \quad$ M. Citterio ${ }^{94 a}$, M. Ciubancan ${ }^{28 b}$, A. Clark ${ }^{52}$, B. L. Clark ${ }^{59}$, M. R. Clark $^{38}$, P. J. Clark ${ }^{49}$, R. N. Clarke ${ }^{16}$, C. Clement ${ }^{148 a, 148 b}$, Y. Coadou ${ }^{88}$, M. Cobal ${ }^{167 a, 167 c}$, A. Coccaro ${ }^{52}$, J. Cochran ${ }^{67}$, L. Colasurdo ${ }^{108}$, B. Cole ${ }^{38}$, A. P. Colijn ${ }^{109}$, J. Collot ${ }^{58}$, T. Colombo ${ }^{166}$, P. Conde Muiño ${ }^{128 a, 128 b}$, E. Coniavitis ${ }^{51}$, S. H. Connell ${ }^{147 b}$, I. A. Connelly ${ }^{87}$, S. Constantinescu ${ }^{28 b}$, G. Conti ${ }^{32}$, F. Conventi ${ }^{106 a, n}$, M. Cooke ${ }^{16}$, A. M. Cooper-Sarkar ${ }^{122}$, F. Cormier ${ }^{171}$, K. J. R. Cormier ${ }^{161}, \quad$ M. Corradi ${ }^{134 a, 134 b}$, F. Corriveau ${ }^{900}$, A. Cortes-Gonzalez ${ }^{32}$, G. Cortiana ${ }^{103}$, G. Costa $^{94 a}$, M. J. Costa ${ }^{170}$, D. Costanzo ${ }^{141}$, G. Cottin ${ }^{30}$, G. Cowan ${ }^{80}$, B. E. $\mathrm{Cox}^{87}$, K. Cranmer ${ }^{112}$, S. J. Crawley ${ }^{56}$, R. A. Creager ${ }^{124}$, G. Cree ${ }^{31}$, S. Crépé-Renaudin ${ }^{58}$, F. Crescioli ${ }^{83}$,

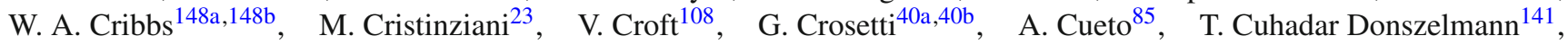
A. R. Cukierman ${ }^{145}$, J. Cummings ${ }^{179}$, M. Curatolo ${ }^{50}$, J. Cúth ${ }^{86}, \quad$ H. Czirr ${ }^{143}$, P. Czodrowski ${ }^{32}$, G. D'amen ${ }^{22 a, 22 b}$, S. D’Auria ${ }^{56}$, L. D'eramo ${ }^{83}$, M. D'Onofrio ${ }^{77}$, M. J. Da Cunha Sargedas De Sousa ${ }^{128 a, 128 b}$, C. Da Via ${ }^{87}$, W. Dabrowski ${ }^{41 a}$, T. Dado ${ }^{146 a}$, T. Dai ${ }^{92}$, O. Dale ${ }^{15}$, F. Dallaire ${ }^{97}$, C. Dallapiccola ${ }^{89}$, M. Dam ${ }^{39}$, J. R. Dandoy ${ }^{124}$, M. F. Daneri ${ }^{29}$, N. P. Dang ${ }^{176}$, A. C. Daniells ${ }^{19}$, N. S. Dann ${ }^{87}$, M. Danninger ${ }^{171}$, M. Dano Hoffmann ${ }^{138}$, V. Dao ${ }^{150}$, G. Darbo ${ }^{53 a}$, S. Darmora ${ }^{8}$, J. Dassoulas ${ }^{3}$, A. Dattagupta ${ }^{118}$, T. Daubney ${ }^{45}$, W. Davey ${ }^{23}$, C. David ${ }^{45}$, T. Davidek ${ }^{131}$, M. Davies ${ }^{155}$, D. R. Davis ${ }^{48}$, P. Davison ${ }^{81}$, E. Dawe ${ }^{91}$, I. Dawson ${ }^{141}$, K. De $^{8}$, R. de Asmundis ${ }^{106 a}$, A. De Benedetti ${ }^{115}$, S. De Castro ${ }^{22 a, 22 b}$, S. De $\mathrm{Cecco}^{83}$, N. De Groot ${ }^{108}$, P. de Jong ${ }^{109}$, H. De la Torre ${ }^{93}$, F. De Lorenzi ${ }^{67}$, A. De Maria ${ }^{57}$, D. De Pedis ${ }^{134 a}$, A. De Salvo ${ }^{134 a}$, U. De Sanctis ${ }^{135 a, 135 b}$, A. De Santo ${ }^{151}$, K. De Vasconcelos Corga ${ }^{88}$ ， J. B. De Vivie De Regie ${ }^{119}$, W. J. Dearnaley ${ }^{75}$, R. Debbe ${ }^{27}$, C. Debenedetti ${ }^{139}$, D. V. Dedovich ${ }^{68}$, N. Dehghanian ${ }^{3}$, I. Deigaard ${ }^{109}$, M. Del Gaudio ${ }^{40 a, 40 b}$, J. Del Peso ${ }^{85}$, T. Del Prete ${ }^{126 a, 126 b}$, D. Delgove ${ }^{119}$, F. Deliot ${ }^{138}$, C. M. Delitzsch ${ }^{52}$, A. Dell'Acqua ${ }^{32}$, L. Dell'Asta ${ }^{24}$, M. Dell'Orso ${ }^{126 a, 126 b}, \quad$ M. Della Pietra106a,106b,$\quad$ D. della Volpe ${ }^{52}, \quad$ M. Delmastro ${ }^{5}$, C. Delporte ${ }^{119}$, P. A. Delsart ${ }^{58}$, D. A. DeMarco ${ }^{161}$, S. Demers ${ }^{179}$, M. Demichev ${ }^{68}$, A. Demilly ${ }^{83}$, S. P. Denisov ${ }^{132}$, D. Denysiuk ${ }^{138}$, D. Derendarz ${ }^{42}$, J. E. Derkaoui ${ }^{137 d}$, F. Derue ${ }^{83}$, P. Dervan ${ }^{77}$, K. $\operatorname{Desch}^{23}$, C. Deterre ${ }^{45}$, K. Dette ${ }^{46}$, M. R. Devesa ${ }^{29}$, P. O. Deviveiros ${ }^{32}$, A. Dewhurst ${ }^{133}$, S. Dhaliwall ${ }^{25}$, F. A. Di Bello ${ }^{52}$, A. Di Ciaccio ${ }^{135 a, 135 b}$, L. Di Ciaccio ${ }^{5}$, W. K. Di Clemente ${ }^{124}$, C. Di Donato ${ }^{106 a, 106 b}$, A. Di Girolamo ${ }^{32}$, B. Di Girolamo ${ }^{32}$, B. Di Micco 136a,136b, R. Di Nardo ${ }^{32}$, K. F. Di Petrillo ${ }^{59}$, A. Di Simone ${ }^{51}$, R. Di Sipio ${ }^{161}$ ， D. Di Valentino ${ }^{31}$, C. Diaconu ${ }^{88}$ ， M. Diamond ${ }^{161}$, F. A. Dias ${ }^{39}$, M. A. Diaz ${ }^{34 a}$, E. B. Diehl ${ }^{92}$, J. Dietrich ${ }^{17}$, S. Díez Cornell ${ }^{45}$, A. Dimitrievska ${ }^{14}$, J. Dingfelder ${ }^{23}$, P. Dita ${ }^{28 b}$, S. Dita ${ }^{28 b}$, F. Dittus ${ }^{32}$, F. Djama ${ }^{88}$, T. Djobava ${ }^{54 b}$, J. I. Djuvsland ${ }^{60 a}$, M. A. B. do Vale ${ }^{26 c}$, D. Dobos ${ }^{32}$, M. Dobre ${ }^{28 b}$, C. Doglioni ${ }^{84}$, J. Dolejsi ${ }^{131}$, Z. Dolezal ${ }^{131}$ ， M. Donadelli ${ }^{26 d}$ ， S. Donati ${ }^{126 a, 126 b}$, P. Dondero ${ }^{123 a, 123 b ， \quad J . ~ D o n i n i ~}{ }^{37}$ ， J. Dopke ${ }^{133}$ ， A. Doria ${ }^{106 a}$, M. T. Dova ${ }^{74}$, A. T. Doyle ${ }^{56}$, E. Drechsler ${ }^{57}$, M. Dris ${ }^{10}$, Y. Du $^{36 b}$, J. Duarte-Campderros ${ }^{155}$, A. Dubreuil ${ }^{52}$, E. Duchovni ${ }^{175}$, G. Duckeck ${ }^{102}$, A. Ducourthial ${ }^{83}$, O. A. Ducu ${ }^{97 p}$, D. Duda ${ }^{109}$, A. Dudarev ${ }^{32}$, A. Chr. Dudder ${ }^{86}$, E. M. Duffield ${ }^{16}$, L. Duflot ${ }^{119}$, M. Dührssen ${ }^{32}$, M. Dumancic ${ }^{175}$, A. E. Dumitriu ${ }^{28 b}$, A. K. Duncan ${ }^{56}$, M. Dunford ${ }^{60 a}$, H. Duran Yildiz ${ }^{4 a}$, 
M. Düren ${ }^{55}$, A. Durglishvili5 ${ }^{54}$, D. Duschinger ${ }^{47}$, B. Dutta ${ }^{45}$, M. Dyndal ${ }^{45}$, C. Eckardt ${ }^{45}$, K. M. Ecker ${ }^{103}$, R. C. Edgar ${ }^{92}$, T. Eifert $^{32}$, G. Eigen ${ }^{15}$, K. Einsweiler ${ }^{16}$, T. Ekelof ${ }^{168}$, M. El Kacimi ${ }^{137 c}$, R. El Kosseifi ${ }^{88}$, V. Ellajosyula ${ }^{88}$, M. Ellert ${ }^{168}$, S. Elles ${ }^{5}$, F. Ellinghaus ${ }^{178}$, A. A. Elliot ${ }^{172}$, N. Ellis $^{32}$, J. Elmsheuser ${ }^{27}$, M. Elsing ${ }^{32}$, D. Emeliyanov ${ }^{133}$, Y. Enari ${ }^{157}$, O. C. Endner ${ }^{86}$, J.S. Ennis ${ }^{173}$ ， J. Erdmann ${ }^{46}$, A. Ereditato ${ }^{18}$, G. Ernis ${ }^{178}$, M. Ernst ${ }^{27}$, S. Errede ${ }^{169}$, M. Escalier ${ }^{119}$, C. Escobar ${ }^{127}$, B. Esposito ${ }^{50}$, O. Estrada Pastor ${ }^{170}$, A. I. Etienvre ${ }^{138}$, E. Etzion ${ }^{155}$, H. Evans ${ }^{64}$, A. Ezhilov ${ }^{125}$, M. Ezzi ${ }^{137 e}$, F. Fabbri22a,22b， L. Fabbri2 ${ }^{2 a, 22 b}$ ， G. Facini ${ }^{33} ， \quad$ R. M. Fakhrutdinov ${ }^{132}$ ， S. Falciano ${ }^{134 a}$ ， R. J. Falla ${ }^{81}$ ， J. Faltova ${ }^{32}$, Y. Fang ${ }^{35 a}$, M. Fanti ${ }^{94 a, 94 b}$, A. Farbin ${ }^{8}$, A. Farilla ${ }^{136 a}$, C. Farina ${ }^{127}$, E. M. Farina ${ }^{123 a, 123 b}$, T. Farooque ${ }^{93}$, S. Farrel1 ${ }^{16}$, S. M. Farrington ${ }^{173}$, P. Farthouat ${ }^{32}$, F. Fassi ${ }^{137 e}$, P. Fassnacht ${ }^{32}$, D. Fassouliotis ${ }^{9}$, M. Faucci Giannelli ${ }^{80}$, A. Favareto ${ }^{53 a, 53 b}$, W. J. Fawcett ${ }^{122}$ ， L. Fayard ${ }^{119}$ ，O. L. Fedin ${ }^{125 q}$ ，W. Fedorko ${ }^{171}$ ，S. Feigl ${ }^{121}$ ， L. Feligioni ${ }^{88}$ ， C. Feng ${ }^{36 b}$ ， E. J. Feng ${ }^{32}$, H. Feng ${ }^{92}$, M. J. Fenton ${ }^{56}$, A. B. Fenyuk ${ }^{132}$, L. Feremenga ${ }^{8}$, P. Fernandez Martinez ${ }^{170}$, S. Fernandez Perez ${ }^{13}$, J. Ferrando ${ }^{45}$, A. Ferrari ${ }^{168}$, P. Ferrari ${ }^{109}$, R. Ferrari ${ }^{123 a}$, D. E. Ferreira de Lima ${ }^{60 b}$, A. Ferrer ${ }^{170}$, D. Ferrere ${ }^{52}$, C. Ferretti ${ }^{92}$, F. Fiedler ${ }^{86}$, A. Filipčič ${ }^{78}$, M. Filipuzzi ${ }^{45}$, F. Filthaut ${ }^{108}$, M. Fincke-Keeler ${ }^{172}$, K. D. Finelli ${ }^{152}$, M. C. N. Fiolhais ${ }^{128 a, 128 c, r}$, L. Fiorini ${ }^{170}$, A. Fischer ${ }^{2}$, C. Fischer ${ }^{13}$, J. Fischer ${ }^{178}$, W. C. Fisher ${ }^{93}$, N. Flaschel ${ }^{45}$, I. Fleck ${ }^{143}$, P. Fleischmann ${ }^{92}$, R. R. M. Fletcher ${ }^{124}$, T. Flick ${ }^{178}$, B. M. Flierl ${ }^{102}$, L. R. Flores Castillo ${ }^{62 a}$, M. J. Flowerdew ${ }^{103}$, G. T. Forcolin ${ }^{87}$, A. Formica ${ }^{138}$, F. A. Förster ${ }^{13}$, A. Forti ${ }^{87}$, A. G. Foster ${ }^{19}$, D. Fournier ${ }^{119}$, H. Fox ${ }^{75}$, S. Fracchia ${ }^{141}$, P. Francavilla ${ }^{83}$, M. Franchini ${ }^{22 a, 22 b}$, S. Franchino ${ }^{60 a}$, D. Francis ${ }^{32}$,L. Franconi ${ }^{121}$, M. Franklin ${ }^{59}$, M. Frate ${ }^{166}$, M. Fraternali ${ }^{123 a, 123 b}$, D. Freeborn ${ }^{81}$, S. M. Fressard-Batraneanu ${ }^{32}$, B. Freund ${ }^{97}$, D. Froidevaux ${ }^{32}$, J. A. Frost ${ }^{122}$, C. Fukunaga ${ }^{158}$, T. Fusayasu ${ }^{104}$, J. Fuster ${ }^{170}$, C. Gabaldon ${ }^{58}$, O. Gabizon ${ }^{154}$, A. Gabrielli22a,22b, A. Gabrielli ${ }^{16}$, G. P. Gach ${ }^{41 a}$, S. Gadatsch ${ }^{32}$, S. Gadomski ${ }^{80}$, G. Gagliardi ${ }^{53 a, 53 b}$, L. G. Gagnon ${ }^{97}$, C. Galea ${ }^{108}$, B. Galhardo ${ }^{128 a}$,128c , E. J. Gallas ${ }^{122}$, B. J. Gallop ${ }^{133}$, P. Gallus ${ }^{130}$, G. Galster ${ }^{39}$, K. K. Gan ${ }^{113}$, S. Ganguly ${ }^{37}$, Y. Gao ${ }^{77}$, Y. S. Gao ${ }^{145 g}$, F. M. Garay Walls ${ }^{49}$, C. García ${ }^{170}$, J. E. García Navarro ${ }^{170}$, M. Garcia-Sciveres ${ }^{16}$, R. W. Gardner ${ }^{33}$, N. Garelli ${ }^{145}$, V. Garonne ${ }^{121}$, A. Gascon Bravo ${ }^{45}$, K. Gasnikova ${ }^{45}$, C. Gatti50, A. Gaudiello ${ }^{53 a, 53 b}$, G. Gaudio ${ }^{123 a}$, I. L. Gavrilenko ${ }^{98}$, C. Gay ${ }^{171}$, G. Gaycken ${ }^{23}$, E. N. Gazis ${ }^{10}$, C. N. P. Gee ${ }^{133}$, J. Geisen ${ }^{57}$, M. Geisen ${ }^{86}$, M. P. Geisler ${ }^{60 a}$, K. Gellerstedt ${ }^{148 a}$,148b , C. Gemme ${ }^{53 a}$, M. H. Genest ${ }^{58}$, C. Geng ${ }^{92}$, S. Gentile ${ }^{134 a, 134 b}$, C. Gentsos ${ }^{156}, \quad$ S. George ${ }^{80}$, D. Gerbaudo ${ }^{13}$, A. Gershon ${ }^{155}$, G. Geßner ${ }^{46}$, S. Ghasemi ${ }^{143}, \quad$ M. Ghneimat ${ }^{23}$, B. Giacobbe ${ }^{22 a}$, S. Giagu ${ }^{134 a, 134 b}$, P. Giannetti ${ }^{126 a, 126 b}$, S. M. Gibson ${ }^{80}$, M. Gignac ${ }^{171}$, M. Gilchriese ${ }^{16}$, D. Gillberg ${ }^{31}$, G. Gilles ${ }^{178}$, D. M. Gingrich ${ }^{3 \mathrm{~d}}$, N. Giokaris ${ }^{9, *}$, M. P. Giordani ${ }^{167 a, 167 c}$, F. M. Giorgi ${ }^{22 a}$, P. F. Giraud ${ }^{138}, \quad$ P. Giromini ${ }^{59}$, D. Giugni ${ }^{94 a}$, F. Giuli ${ }^{122}$, C. Giuliani ${ }^{103}$, M. Giulini ${ }^{60 b}$, B. K. Gjelsten ${ }^{121}$, S. Gkaitatzis ${ }^{156}$, I. Gkialas ${ }^{9}$, E. L. Gkougkousis ${ }^{139}$, P. Gkountoumis ${ }^{10}$, L. K. Gladilin ${ }^{101}$, C. Glasman ${ }^{85}$, J. Glatzer ${ }^{13}$, P. C. F. Glaysher ${ }^{45}$, A. Glazov ${ }^{45}$, M. Goblirsch-Kolb ${ }^{25}$, J. Godlewski ${ }^{42}$, S. Goldfarb ${ }^{91}$, T. Golling ${ }^{52}$, D. Golubkov ${ }^{132}$, A. Gomes ${ }^{128 a, 128 b, 128 d}$, R. Gonçalo ${ }^{128 a}$, R. Goncalves Gama ${ }^{26 a}$, $\begin{array}{lll}\text { J. Goncalves Pinto Firmino Da Costa } & 138\end{array}$ G. Gonella ${ }^{51}$, L. Gonella ${ }^{19}$, A. Gongadze ${ }^{68}$, S. González de la Hoz ${ }^{170}$, S. Gonzalez-Sevilla ${ }^{52}$, L. Goossens ${ }^{32}$, P. A. Gorbounov ${ }^{99}$, H. A. Gordon ${ }^{27}$, I. Gorelov ${ }^{107}$, B. Gorini ${ }^{32}$, E. Gorini ${ }^{76 a, 76 b}$, A. Gorišek ${ }^{78}$, A. T. Goshaw ${ }^{48}$, C. Gössling ${ }^{46}$, M. I. Gostkin ${ }^{68}$, C. A. Gottardo ${ }^{23}$, C. R. Goudet ${ }^{119}$, D. Goujdami ${ }^{137 c}$, A. G. Goussiou ${ }^{140}$, N. Govender ${ }^{147 b, s}$, E. Gozani ${ }^{154}$, L. Graber ${ }^{57}$, I. Grabowska-Bold ${ }^{41 a}$, P. O. J. Gradin ${ }^{168}$, J. Gramling ${ }^{166}$, E. Gramstad ${ }^{121}$, S. Grancagnolo ${ }^{17}$, V. Gratchev ${ }^{125}$, P. M. Gravila ${ }^{28 f}$, C. Gray ${ }^{56}$, H. M. Gray ${ }^{16}$, Z. D. Greenwood ${ }^{82 t}$, C. Grefe $^{23}$, K. Gregersen ${ }^{81}$, I. M. Gregor ${ }^{45}$, P. Grenier ${ }^{145}$, K. Grevtsov ${ }^{5}$, J. Griffiths ${ }^{8}$, A. A. Grillo ${ }^{139}$, K. Grimm ${ }^{75}$, S. Grinstein ${ }^{13 \mathrm{u}}$, Ph. Gris ${ }^{37}$, J.-F. Grivaz ${ }^{119}$, S. Groh ${ }^{86}$, E. Gross ${ }^{175}$, J. Grosse-Knetter ${ }^{57}$, G. C. Grossi ${ }^{82}$, Z. J. Grout ${ }^{81}$, A. Grummer ${ }^{107}$, L. Guan ${ }^{92}$, W. Guan ${ }^{176}$, J. Guenther ${ }^{65}$, F. Guescini163a, D. Guest ${ }^{166}$, O. Gueta ${ }^{155}$, B. Gui ${ }^{113}$, E. Guido ${ }^{53 a, 53 b}$, T. Guillemin ${ }^{5}$, S. Guindon ${ }^{2}$, U. Gul ${ }^{56}$, C. Gumpert ${ }^{32}$, J. Guo ${ }^{36 c}$, W. Guo ${ }^{92}$, Y. Guo ${ }^{36 a}$, R. Gupta ${ }^{43}$, S. Gupta ${ }^{122}$, G. Gustavino ${ }^{134 a, 134 b}$, P. Gutierrez ${ }^{115}$, N. G. Gutierrez Ortiz ${ }^{81}$, C. Gutschow ${ }^{81}$, C. Guyot ${ }^{138}$, M. P. Guzik ${ }^{41 a}$,

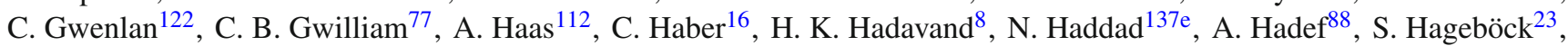
M. Hagihara ${ }^{164}$, H. Hakobyan ${ }^{180, *}$, M. Haleem ${ }^{45}$, J. Haley ${ }^{116}$, G. Halladjian ${ }^{93}$, G. D. Hallewell ${ }^{88}$, K. Hamacher ${ }^{178}$, P. Hamal ${ }^{117}$, K. Hamano ${ }^{172}$, A. Hamilton ${ }^{147 a}$, G. N. Hamity ${ }^{141}$, P. G. Hamnett ${ }^{45}$, L. Han ${ }^{36 a}$, S. Han ${ }^{35 a}$, K. Hanagaki ${ }^{69 v}$, K. Hanawa ${ }^{157}$, M. Hance ${ }^{139}$, B. Haney ${ }^{124}$, P. Hanke ${ }^{60 a}$, J. B. Hansen ${ }^{39}$, J. D. Hansen ${ }^{39}$, M. C. Hansen ${ }^{23}$, P. H. Hansen ${ }^{39}$, K. Hara ${ }^{164}$, A. S. Hard ${ }^{176}$, T. Harenberg ${ }^{178}$, F. Hariri ${ }^{119}$, S. Harkusha ${ }^{95}$, R. D. Harrington ${ }^{49}$, P. F. Harrison ${ }^{173}$, N. M. Hartmann ${ }^{102}$, M. Hasegawa ${ }^{70}$, Y. Hasegawa ${ }^{142}$, A. Hasib ${ }^{49}$, S. Hassani ${ }^{138}$, S. Haug ${ }^{18}$, R. Hauser ${ }^{93}$, L. Hauswald ${ }^{47}$, L. B. Havener ${ }^{38}$, M. Havranek ${ }^{130}$, C. M. Hawkes ${ }^{19}$, R. J. Hawkings ${ }^{32}$, D. Hayakawa ${ }^{159}$, D. Hayden ${ }^{93}$, C. P. Hays ${ }^{122}$,

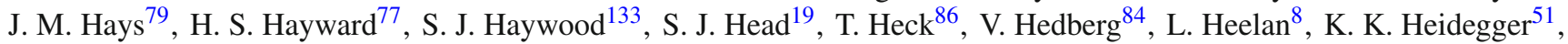
S. Heim ${ }^{45}$, T. Heim ${ }^{16}$, B. Heinemann ${ }^{45 w}$, J. J. Heinrich ${ }^{102}$, L. Heinrich ${ }^{112}$, C. Heinz ${ }^{55}$, J. Hejbal ${ }^{129}$, L. Helary ${ }^{32}$, A. Held ${ }^{171}$, S. Hellman ${ }^{148 a}$, 148b, C. Helsens ${ }^{32}$, R. C. W. Henderson ${ }^{75}$, Y. Heng ${ }^{176}$, S. Henkelmann ${ }^{171}$, A. M. Henriques Correia ${ }^{32}$, S. Henrot-Versille ${ }^{119}$ ， G. H. Herbert ${ }^{17}$ ， H. Herde ${ }^{25}$, V. Herget ${ }^{177}$, Y. Hernández Jiménez ${ }^{147 c}$ ，H. Herr ${ }^{86}$, G. Herten ${ }^{51}$, R. Hertenberger ${ }^{102}$, L. Hervas ${ }^{32}$, T. C. Herwig ${ }^{124}$, G. G. Hesketh ${ }^{81}$, N. P. Hessey ${ }^{163 a}$, J. W. Hetherly ${ }^{43}$, S. Higashino ${ }^{69}$, E. Higón-Rodriguez ${ }^{170}$, E. Hill ${ }^{172}$, J. C. Hill ${ }^{30}$, K. H. Hiller ${ }^{45}$, S. J. Hillier ${ }^{19}$, M. Hils ${ }^{47}$, I. Hinchliffe ${ }^{16}$, M. Hirose ${ }^{51}$,

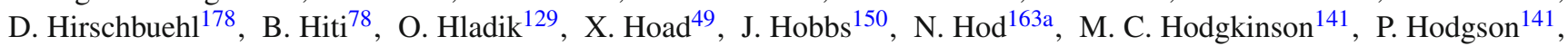


A. Hoecker ${ }^{32}$, M. R. Hoeferkamp ${ }^{107}$, F. Hoenig ${ }^{102}$, D. Hohn ${ }^{23}$, T. R. Holmes ${ }^{33}$, M. Homann ${ }^{46}$, S. Honda ${ }^{164}$, T. Honda ${ }^{69}$, T. M. Hong ${ }^{127}$, B. H. Hooberman ${ }^{169}$ ， W. H. Hopkins ${ }^{118}$, Y. Horii ${ }^{105}$ ， A. J. Horton ${ }^{144}$, J.-Y. Hostachy ${ }^{58}$, S. Hou ${ }^{153}$, A. Hoummada ${ }^{137 a}$, J. Howarth ${ }^{87}$, J. Hoya ${ }^{74}$, M. Hrabovsky ${ }^{117}$, J. Hrdinka ${ }^{32}$, I. Hristova ${ }^{17}$, J. Hrivnac ${ }^{119}$, T. Hryn'ova ${ }^{5}$, A. Hrynevich ${ }^{96}$, P. J. Hsu ${ }^{63}$, S.-C. $\mathrm{Hsu}^{140}$, Q. Hu ${ }^{36 a}$, S. Hu ${ }^{36 c}$, Y. Huang ${ }^{35 a}$, Z. Hubacek ${ }^{130}$, F. Hubaut ${ }^{88}$, F. Huegging ${ }^{23}$, T. B. Huffman ${ }^{122}$, E. W. Hughes ${ }^{38}$, G. Hughes ${ }^{75}$, M. Huhtinen ${ }^{32}$, P. Huo ${ }^{150}$, N. Huseynov ${ }^{68 b}$, J. Huston ${ }^{93}$, J. Huth ${ }^{59}$, G. Iacobucci ${ }^{52}$ ，G. Iakovidis ${ }^{27}$ ，I. Ibragimov ${ }^{143}$ ，L. Iconomidou-Fayard ${ }^{119}$ ，Z. Idrissi ${ }^{137 e}$ ，P. Iengo ${ }^{32}$ ，O. Igonkina ${ }^{109 x}$, T. Iizawa ${ }^{174}$, Y. Ikegami ${ }^{69}$ ， M. Ikeno ${ }^{69}$ ，Y. Ilchenko ${ }^{11 y}$ ，D. Iliadis ${ }^{156}$ ，N. Ilic ${ }^{145}$ ，G. Introzzi ${ }^{123 a, 123 b}$ ， P. Ioannou ${ }^{9, *}$, M. Iodice ${ }^{136 a}$, K. Iordanidou ${ }^{38}$, V. Ippolito ${ }^{59}$, M. F. Isacson ${ }^{168}$, N. Ishijima ${ }^{120}$, M. Ishino ${ }^{157}$, M. Ishitsuka ${ }^{159}$, C. Issever ${ }^{122}$, S. Istin ${ }^{20 a}$, F. Ito ${ }^{164}$, J. M. Iturbe Ponce ${ }^{87}$, R. Iuppa ${ }^{162 a, 162 b}$, H. Iwasaki ${ }^{69}$, J. M. Izen ${ }^{44}$, V. Izzo ${ }^{106 a}$, S. Jabbar ${ }^{3}$, P. Jackson ${ }^{1}$, R. M. Jacobs ${ }^{23}$ ，V. Jain ${ }^{2}$ ，K. B. Jakobi ${ }^{86}$ ，K. Jakobs ${ }^{51}$ ， S. Jakobsen ${ }^{65}$ ，T. Jakoubek ${ }^{129}$ ，D. O. Jamin ${ }^{116}$ ， D. K. Jana ${ }^{82}$, R. Jansky ${ }^{52}$ ， J. Janssen ${ }^{23}$ ， M. Janus ${ }^{57 ｝ \text { ，P. A. Janus }{ }^{41 a} \text { ， G. Jarlskog }{ }^{84} \text { ， N. Javadov }{ }^{68 b} \text { ， T. Javůrek }{ }^{51 ， \quad M . ~ J a v u r k o v a ~}{ }^{51} \text {, }$ F. Jeanneau ${ }^{138}$, L. Jeanty ${ }^{16}$, J. Jejelava ${ }^{54 a, z}$, A. Jelinskas ${ }^{173}$, P. Jenni ${ }^{51, a a}$, C. Jeske ${ }^{173}$, S. Jézéquel ${ }^{5}$, H. Ji ${ }^{176}$, J. Jia ${ }^{150}$, H. Jiang ${ }^{67}$, Y. Jiang ${ }^{36 a}$, Z. Jiang ${ }^{145}$, S. Jiggins ${ }^{81}$, J. Jimenez Pena ${ }^{170}$, S. Jin ${ }^{35 a}$, A. Jinaru ${ }^{28 b}$, O. Jinnouchi ${ }^{159}$, H. Jivan ${ }^{147 c}$, P. Johansson ${ }^{141}$, K. A. Johns ${ }^{7}$, C. A. Johnson ${ }^{64}$, W. J. Johnson ${ }^{140}$, K. Jon-And ${ }^{148 a, 148 b}$, R. W. L. Jones ${ }^{75}$, S. D. Jones ${ }^{151}$, S. Jones ${ }^{7}$, T. J. Jones ${ }^{77}$, J. Jongmanns ${ }^{60 a}$, P. M. Jorge ${ }^{128 a, 128 b}$, J. Jovicevic ${ }^{163 a}$, X. Ju ${ }^{176}$, A. Juste Rozas ${ }^{13 u}$, M. K. Köhler ${ }^{175}$, A. Kaczmarska ${ }^{42}$, M. Kado ${ }^{119}$, H. Kagan ${ }^{113}$, M. Kagan ${ }^{145}$, S. J. Kahn ${ }^{88}$, T. Kaji ${ }^{174}$, E. Kajomovitz ${ }^{48}$, C. W. Kalderon ${ }^{84}$, A. Kaluza ${ }^{86}$, S. Kama ${ }^{43}$, A. Kamenshchikov ${ }^{132}$, N. Kanaya ${ }^{157}$, L. Kanjir ${ }^{78}$, V. A. Kantserov ${ }^{100}$, J. Kanzaki ${ }^{69}$, B. Kaplan ${ }^{112}$, L. S. Kaplan ${ }^{176}$, D. Kar ${ }^{147 c}$, K. Karakostas ${ }^{10}$, N. Karastathis ${ }^{10}$, M. J. Kareem ${ }^{57}$ ， E. Karentzos ${ }^{10}$, S. N. Karpov ${ }^{68}$, Z. M. Karpova ${ }^{68}$, K. Karthik ${ }^{112}$, V. Kartvelishvili ${ }^{75}$, A. N. Karyukhin ${ }^{132}$, K. Kasahara ${ }^{164}$, L. Kashif ${ }^{176}$, R. D. Kass ${ }^{113}$, A. Kastanas ${ }^{149}$, Y. Kataoka ${ }^{157}$, C. Kato ${ }^{157}$, A. Katre ${ }^{52}$, J. Katzy ${ }^{45}$, K. Kawade ${ }^{70}$, K. Kawagoe ${ }^{73}$, T. Kawamoto ${ }^{157}$, G. Kawamura ${ }^{57}$, E. F. Kay ${ }^{77}$, V. F. Kazanin ${ }^{111 c}$, R. Keeler ${ }^{172}$, R. Kehoe ${ }^{43}$, J. S. Keller ${ }^{31}$, J. J. Kempster ${ }^{80}$, J Kendrick ${ }^{19}$, H. Keoshkerian ${ }^{161}$, O. Kepka ${ }^{129}$, B. P. Kerševan ${ }^{78}$, S. Kersten ${ }^{178}$, R. A. Keyes ${ }^{90}$, M. Khader ${ }^{169}$, F. Khalil-zada ${ }^{12}$, A. Khanov ${ }^{116}$, A. G. Kharlamov ${ }^{111 c}$, T. Kharlamova ${ }^{111 c}$, A. Khodinov ${ }^{160}$, T. J. Khoo ${ }^{52}$, V. Khovanskiy ${ }^{99, *}$, E. Khramov ${ }^{68}$, J. Khubua ${ }^{54 b, a b}$, S. Kido ${ }^{70}$, C. R. Kilby ${ }^{80}$, H. Y. Kim ${ }^{8}$, S. H. Kim ${ }^{164}$, Y. K. Kim ${ }^{33}$, N. Kimura ${ }^{156}$, O. M. Kind ${ }^{17}$, $\begin{array}{llll}\text { B. T. King } & 77 \text {, D. Kirchmeier } \\ 47 & \text { J. Kirk }\end{array}{ }^{133}$, A. E. Kiryunin ${ }^{103}$, T. Kishimoto ${ }^{157}$, D. Kisielewska41a, V. Kitali ${ }^{45}$, K. Kiuchi ${ }^{164}$, O. Kivernyk ${ }^{5}$, E. Kladiva ${ }^{146 b}$, T. Klapdor-Kleingrothaus ${ }^{51}$, M. H. Klein ${ }^{38}$, M. Klein ${ }^{77}$, U. Klein ${ }^{77}$, K. Kleinknecht ${ }^{86}$, P. Klimek ${ }^{110}$, A. Klimentov ${ }^{27}$, R. Klingenberg ${ }^{46}$, T. Klingl ${ }^{23}$, T. Klioutchnikova ${ }^{32}$, E.-E. Kluge ${ }^{60 a}$,

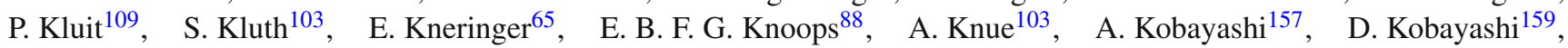
T. Kobayashi ${ }^{157}$, M. Kobel ${ }^{47}$, M. Kocian ${ }^{145}$, P. Kodys ${ }^{131}$, T. Koffas ${ }^{31}$, E. Koffeman ${ }^{109}$, N. M. Köhler ${ }^{103}$, T. Koi ${ }^{145}$, M. Kolb ${ }^{60 b}$, I. Koletsou ${ }^{5}$, A. A. Komar ${ }^{98, *}$, Y. Komori ${ }^{157}$, T. Kondo ${ }^{69}$, N. Kondrashova ${ }^{36 c}$, K. Köneke ${ }^{51}$, A. C. König ${ }^{108}$, T. Kono ${ }^{69}$,ac , R. Konoplich ${ }^{112, \text { ad }}$, N. Konstantinidis ${ }^{81}$, R. Kopeliansky ${ }^{64}$, S. Koperny ${ }^{41 a}$, A. K. Kopp ${ }^{51}$, K. Korcyl ${ }^{42}$, K. Kordas ${ }^{156}$, A. Korn ${ }^{81}$, A. A. Korol ${ }^{111}$, I. Korolkov ${ }^{13}$, E. V. Korolkova ${ }^{141}$, O. Kortner ${ }^{103}$, S. Kortner ${ }^{103}$, T. Kosek ${ }^{131}$, V. V. Kostyukhin ${ }^{23}$, A. Kotwal ${ }^{48}$, A. Koulouris ${ }^{10}$, A. Kourkoumeli-Charalampidi ${ }^{123 a, 123 b}$, C. Kourkoumelis ${ }^{9}$, E. Kourlitis ${ }^{141}$, V. Kouskoura ${ }^{27}$, A. B. Kowalewska ${ }^{42}$, R. Kowalewski ${ }^{172}$, T. Z. Kowalski ${ }^{41 a}$ ， C. Kozakai ${ }^{157}$, W. Kozanecki ${ }^{138}$, A. S. Kozhin ${ }^{132}$, V. A. Kramarenko ${ }^{101}$, G. Kramberger ${ }^{78}$, D. Krasnopevtsev ${ }^{100}$, M. W. Krasny ${ }^{83}$, A. Krasznahorkay ${ }^{32}$, D. Krauss ${ }^{103}$, J. A. Kremer ${ }^{41 a}$, J. Kretzschmar ${ }^{77}$, K. Kreutzfeldt ${ }^{55}$, P. Krieger ${ }^{161}$, K. Krizka ${ }^{33}$, K. Kroeninger ${ }^{46}$, H. Kroha ${ }^{103}$, J. Kroll ${ }^{129}$, J. Kroll ${ }^{124}$, J. Kroseberg ${ }^{23}$, J. Krstic ${ }^{14}$, U. Kruchonak ${ }^{68}$, H. Krüger²3, N. Krumnack ${ }^{67}$, M. C. Kruse $^{48}$, T. Kubota ${ }^{91}$, H. Kucuk ${ }^{81}$, S. Kuday ${ }^{4 b}$, J. T. Kuechler ${ }^{178}$, S. Kuehn ${ }^{32}$, A. Kugel ${ }^{60 c}$, F. Kuger ${ }^{177}$, T. Kuhl ${ }^{45}$, V. Kukhtin ${ }^{68}$, R. Kukla ${ }^{88}$, Y. Kulchitsky ${ }^{95}$, S. Kuleshov ${ }^{34 b}$, Y. P. Kulinich ${ }^{169}$, M. Kuna ${ }^{134 a, 134 b}$, T. Kunigo ${ }^{71}$, A. Kupco ${ }^{129}$, T. Kupfer ${ }^{46}$, O. Kuprash ${ }^{155}$, H. Kurashige ${ }^{70}$, L. L. Kurchaninov ${ }^{163 a}$ ， Y. A. Kurochkin ${ }^{95}$ ， M. G. Kurth ${ }^{35 a}$, V. Kus ${ }^{129}$, E. S. Kuwertz ${ }^{172}$, M. Kuze ${ }^{159}$, J. Kvita ${ }^{117}$, T. Kwan ${ }^{172}$, D. Kyriazopoulos ${ }^{141}$, A. La Rosa ${ }^{103}$, J. L. La Rosa Navarro ${ }^{26 d}$, L. La Rotonda ${ }^{40 a}$,40b, C. Lacasta ${ }^{170}$, F. Lacava ${ }^{134 a, 134 b}$, J. Lacey ${ }^{45}$, H. Lacker ${ }^{17}$, D. Lacour ${ }^{83}$, E. Ladygin ${ }^{68}$, R. Lafaye ${ }^{5}$, B. Laforge ${ }^{83}$, T. Lagouri ${ }^{179}$, S. Lai ${ }^{57}$, S. Lammers ${ }^{64}$, W. Lampl ${ }^{7}$, E. Lançon ${ }^{27}$, U. Landgraf ${ }^{51}$, M. P. J. Landon ${ }^{79}$, M. C. Lanfermann ${ }^{52}$, V. S. Lang ${ }^{60 a}$, J. C. Lange ${ }^{13}$, R. J. Langenberg ${ }^{32}$, A. J. Lankford ${ }^{166}$, F. Lanni ${ }^{27}$, K. Lantzsch ${ }^{23}$, A. Lanza ${ }^{123 a}$, A. Lapertosa ${ }^{53 a, 53 b}$, S. Laplace ${ }^{83}$, J. F. Laporte ${ }^{138}$, T. Lari94a ${ }^{94}$ F. Lasagni Manghi ${ }^{22 a, 22 b}$, M. Lassnig ${ }^{32}$, P. Laurelli ${ }^{50}$, W. Lavrijsen ${ }^{16}$, A. T. Law ${ }^{139}$, P. Laycock ${ }^{77}$, T. Lazovich ${ }^{59}$, M. Lazzaroni ${ }^{94 a}$,94b , B. Le ${ }^{91}$, O. Le Dortz ${ }^{83}$, E. Le Guirriec ${ }^{88}$, E. P. Le Quilleuc ${ }^{138}$, M. LeBlanc ${ }^{172}$, T. LeCompte $^{6}$, F. Ledroit-Guillon ${ }^{58}$, C. A. Lee ${ }^{27}$, G. R. Lee ${ }^{133, \text { ae }}$,

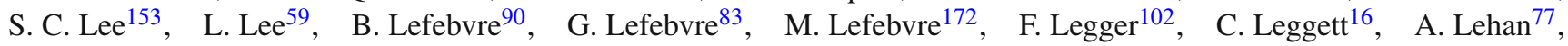
G. Lehmann Miotto ${ }^{32}$ ， X. Lei ${ }^{7}$ ， W. A. Leight ${ }^{45}, \quad$ M. A. L. Leite ${ }^{26 d}$, R. Leitner ${ }^{131}$ ， D. Lellouch ${ }^{175}$, B. Lemmer ${ }^{57}$, K. J. C. Leney ${ }^{81}$, T. Lenz ${ }^{23}$, B. Lenzi ${ }^{32}$, R. Leone ${ }^{7}$, S. Leone ${ }^{126 a, 126 b}$, C. Leonidopoulos ${ }^{49}$, G. Lerner ${ }^{151}$, C. Leroy ${ }^{97}$, A. A. J. Lesage ${ }^{138}$, C. G. Lester ${ }^{30}$, M. Levchenko ${ }^{125}$, J. Levêque ${ }^{5}$, D. Levin ${ }^{92}$, L. J. Levinson ${ }^{175}$, M. Levy ${ }^{19}$, D. Lewis ${ }^{79}$,

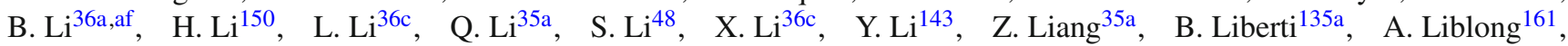
K. Lie ${ }^{62 c}$, J. Liebal ${ }^{23}$, W. Liebig ${ }^{15}$, A. Limosani ${ }^{152}$, S. C. Lin $^{183}$, T. H. Lin ${ }^{86}$, B. E. Lindquist ${ }^{150}$, A. E. Lionti ${ }^{52}$, 


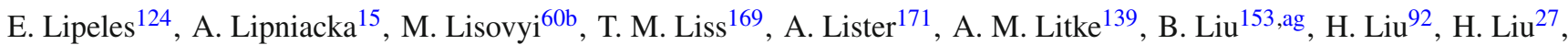

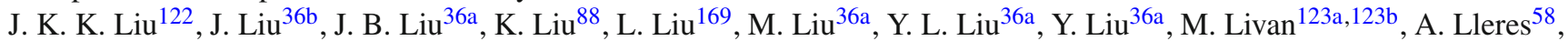
J. Llorente Merino ${ }^{35 a}$, S. L. Lloyd ${ }^{79}$, C. Y. Lo ${ }^{62 b}$, F. Lo Sterzo ${ }^{153}$, E. M. Lobodzinska ${ }^{45}$, P. Loch ${ }^{7}$, F. K. Loebinger ${ }^{87}$, A. Loesle ${ }^{51}$, K. M. Loew ${ }^{25}$, A. Loginov ${ }^{179, *}$, T. Lohse ${ }^{17}$, K. Lohwasser ${ }^{45}$, M. Lokajicek ${ }^{129}$, B. A. Long ${ }^{24}$, J. D. Long ${ }^{169}$, R. E. Long ${ }^{75}$, L. Longo ${ }^{76 a, 76 b}$, K. A. Looper ${ }^{113}$, J. A. Lopez ${ }^{34 b}$, D. Lopez Mateos ${ }^{59}$, I. Lopez Paz ${ }^{13}$, A. Lopez Solis ${ }^{83}$, J. Lorenz ${ }^{102}$ ， N. Lorenzo Martinez ${ }^{5}$ M. Losada ${ }^{21}$ ， P. J. Lösel ${ }^{102}$ ，X. Lou ${ }^{35 a}$ ，A. Lounis ${ }^{119}$ ， J. Love ${ }^{6}$, P. A. Love ${ }^{75}$, H. Lu ${ }^{62 a}$, N. Lu ${ }^{92}$, Y. J. Lu ${ }^{63}$, H. J. Lubatti ${ }^{140}$, C. Luci ${ }^{134 a, 134 b}$, A. Lucotte ${ }^{58}$, C. Luedtke ${ }^{51}$, F. Luehring ${ }^{64}$, W. Lukas ${ }^{65}$, L. Luminari134a, O. Lundberg ${ }^{148 a, 148 b}$, B. Lund-Jensen ${ }^{149}, \quad$ P. M. Luzi ${ }^{83} ， \quad$ D. Lynn ${ }^{27} ， \quad$ R. Lysak ${ }^{129}$, E. Lytken ${ }^{84}$, V. Lyubushkin ${ }^{68}$, H. Ma ${ }^{27}$, L. L. Ma ${ }^{36 b}$, Y. Ma ${ }^{36 b}$, G. Maccarrone ${ }^{50}$, A. Macchiolo ${ }^{103}$, C. M. Macdonald ${ }^{141}$, B. Maček ${ }^{78}$,

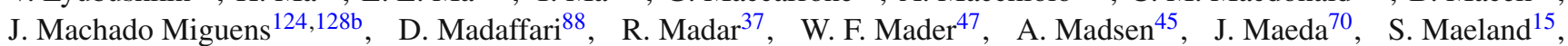
T. Maeno ${ }^{27}$, A. S. Maevskiy ${ }^{101}$, V. Magerl ${ }^{57}$, J. Mahlstedt ${ }^{109}$, C. Maiani ${ }^{119}$, C. Maidantchik ${ }^{26 a}$, A. A. Maier ${ }^{103}$, T. Maier ${ }^{102}$, A. Maio ${ }^{128 a, 128 b, 128 d}$, O. Majersky ${ }^{146 a}$, S. Majewski ${ }^{118}$, Y. Makida ${ }^{69}$, N. Makovec ${ }^{119}$, B. Malaescu ${ }^{83}$, Pa. Malecki ${ }^{42}$, V. P. Maleev ${ }^{125}$, F. Malek ${ }^{58}$, U. Mallik ${ }^{66}$, D. Malon ${ }^{6}$, C. Malone ${ }^{30}$, S. Maltezos ${ }^{10}$, S. Malyukov ${ }^{32}$, J. Mamuzic ${ }^{170}$, G. Mancini ${ }^{50}$, L. Mandelli94a, I. Mandićc78, J. Maneira ${ }^{128 a, 128 b}$, L. Manhaes de Andrade Filho ${ }^{26 \mathrm{~b}}$, J. Manjarres Ramos ${ }^{47}$, A. Mann ${ }^{102}$, A. Manousos ${ }^{32}$, B. Mansoulie ${ }^{138}$, J. D. Mansour ${ }^{35 a}$, R. Mantifel ${ }^{90}$, M. Mantoani ${ }^{57}$, S. Manzoni ${ }^{94 a, 94 b}$, L. Mapelli ${ }^{32}$, G. Marceca ${ }^{29}$, L. March ${ }^{52}$, L. Marchese ${ }^{122}$, G. Marchiori ${ }^{83}$, M. Marcisovsky ${ }^{129}$, M. Marjanovic ${ }^{37}$,

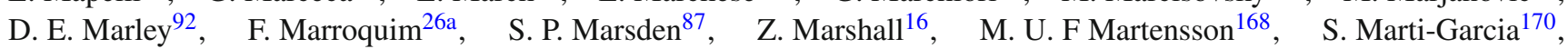
C. B. Martin ${ }^{113}$, T. A. Martin ${ }^{173}$, V. J. Martin ${ }^{49}$, B. Martin dit Latour ${ }^{15}$, M. Martinez ${ }^{13 u}$, V. I. Martinez Outs choorn ${ }^{169}$, S. Martin-Haugh ${ }^{133}$, V. S. Martoiu ${ }^{28 b}$, A. C. Martyniuk ${ }^{81}$, A. Marzin ${ }^{32}$, L. Masetti ${ }^{86}$, T. Mashimo ${ }^{157}$, R. Mashinistov ${ }^{98}$, J. Masik ${ }^{87}$, A. L. Maslennikov ${ }^{111}$, L. Massa ${ }^{135 a, 135 b}$, P. Mastrandrea ${ }^{5}$, A. Mastroberardino ${ }^{40 a, 40 b}$, T. Masubuchi ${ }^{157}$, P. Mättig ${ }^{178}$, J. Maurer ${ }^{28 b}$ ， S. J. Maxfield ${ }^{77}$, D. A. Maximov111c, R. Mazini153, I. Maznas ${ }^{156}$, S. M. Mazza ${ }^{94 a, 94 b}$,

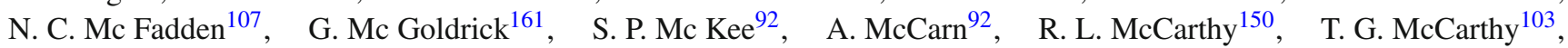
L. I. McClymont ${ }^{81}$, E. F. McDonald ${ }^{91}$, J. A. Mcfayden ${ }^{81}$, G. Mchedlidze ${ }^{57}$, S. J. McMahon ${ }^{133}$, P. C. McNamara ${ }^{91}$, R. A. McPherson ${ }^{1720}, \quad$ S. Meehan ${ }^{140}, \quad$ T. J. Megy ${ }^{51}, \quad$ S. Mehlhase ${ }^{102}, \quad$ A. Mehta ${ }^{77}, \quad$ T. Meideck ${ }^{58}, \quad$ K. Meier ${ }^{60 a}$, B. Meirose ${ }^{44}$, D. Melini ${ }^{170, a h}$, B. R. Mellado Garcia ${ }^{147 c}$, J. D. Mellenthin ${ }^{57}$, M. Melo ${ }^{146 a}$, F. Meloni ${ }^{18}$, S. B. Menary ${ }^{87}$, L. Meng ${ }^{77}$, X. T. Meng ${ }^{92}$, A. Mengarelli2a,22b, S. Menke ${ }^{103}$, E. Meoni ${ }^{40 a, 40 b}$, S. Mergelmeyer ${ }^{17}$, P. Mermod ${ }^{52}$, L. Merola ${ }^{106 a, 106 b}$, C. Meroni ${ }^{94 a}$, F. S. Merritt ${ }^{33}$, A. Messina ${ }^{134 a, 134 b}$, J. Metcalfe ${ }^{6}$, A. S. Mete ${ }^{166}, \quad$ C. Meyer ${ }^{124}$, J.-P. Meyer ${ }^{138}$, J. Meyer ${ }^{109}$, H. Meyer Zu Theenhausen ${ }^{60 a}$, F. Miano ${ }^{151}$, R. P. Middleton ${ }^{133}$, S. Miglioranzi ${ }^{53 a, 53 b}$, L. Mijović49, G. Mikenberg ${ }^{175}$, M. Mikestikova ${ }^{129}$, M. Mikuž ${ }^{78}$, M. Milesi ${ }^{91}$, A. Milic ${ }^{161}$, D. W. Miller ${ }^{33}$, C. Mills ${ }^{49}$, A. Milov $^{175}$, D. A. Milstead ${ }^{148 a, 148 b}$, A. A. Minaenko ${ }^{132}$, Y. Minami ${ }^{157}$, I. A. Minashvili ${ }^{68}$, A. I. Mincer ${ }^{12}$, B. Mindur ${ }^{41 a}$, M. Mineev ${ }^{68}$, Y. Minegishi ${ }^{157}$, Y. Ming ${ }^{176}$, L. M. Mir $^{13}$, K. P. Mistry ${ }^{124}$, T. Mitani ${ }^{174}$, J. Mitrevski ${ }^{102}$, V. A. Mitsou ${ }^{170}$, A. Miucci ${ }^{18}$, P. S. Miyagawa ${ }^{141}$, A. Mizukami ${ }^{69}$, J. U. Mjörnmark ${ }^{84}$, T. Mkrtchyan ${ }^{180}$, M. Mlynarikova ${ }^{131}$, T. Moa ${ }^{148 a, 148 b}$, K. Mochizuki ${ }^{97}$, P. Mogg ${ }^{51}$, S. Mohapatra ${ }^{38}$, S. Molander ${ }^{148 a}$,148b, R. Moles-Valls ${ }^{23}$, R. Monden ${ }^{71}$, M. C. Mondragon ${ }^{93}$, K. Mönig ${ }^{45}$, J. Monk ${ }^{39}$, E. Monnier ${ }^{88}$, A. Montalbano ${ }^{150}$, J. Montejo Berlingen ${ }^{32}$, F. Monticelli ${ }^{74}$, S. Monzani ${ }^{94 a, 94 b}$, R. W. Moore ${ }^{3}$, N. Morange ${ }^{119}$, D. Moreno ${ }^{21}$, M. Moreno Llácer ${ }^{32}$, P. Morettini ${ }^{53 a}$, S. Morgenstern ${ }^{32}$, D. Mori ${ }^{144}$, T. Mori ${ }^{157}$, M. Morii ${ }^{59}$, M. Morinaga ${ }^{157}$, V. Morisbak ${ }^{121}$, A. K. Morley ${ }^{32}$, G. Mornacchi ${ }^{32}$, J. D. Morris ${ }^{79}$, L. Morvaj ${ }^{150}$, P. Moschovakos ${ }^{10}$, M. Mosidze ${ }^{54 b}$, H. J. Moss ${ }^{141}$, J. Moss ${ }^{145, \text { ai }}$, K. Motohashi ${ }^{159}$, R. Mount ${ }^{145}$, E. Mountricha ${ }^{27}$, E. J. W. Moyse ${ }^{89}$, S. Muanza ${ }^{88}$, R. D. Mudd ${ }^{19}$, F. Mueller ${ }^{103}$, J. Mueller ${ }^{127}$, R. S. P. Mueller ${ }^{102}$, D. Muenstermann ${ }^{75}$, P. Mullen $^{56}$, G. A. Mullier ${ }^{18}$, F. J. Munoz Sanchez ${ }^{87}$, W. J. Murray ${ }^{173,133}$, H. Musheghyan ${ }^{181}$, M. Muškinja ${ }^{78}$, A. G. Myagkov ${ }^{132, a j}$, M. Myska ${ }^{130}$, B. P. Nachman ${ }^{16}$, O. Nackenhorst ${ }^{52}$, K. Nagai ${ }^{122}$, R. Nagai ${ }^{69 a, c}$, K. Nagano ${ }^{69}$, Y. Nagasaka ${ }^{61}$, K. Nagata ${ }^{164}$, M. Nagel ${ }^{51}$, E. Nagy ${ }^{88}$, A. M. Nairz ${ }^{32}$, Y. Nakahama ${ }^{105}$, K. Nakamura ${ }^{69}$, T. Nakamura ${ }^{157}$, I. Nakano ${ }^{114}$, R. F. Naranjo Garcia ${ }^{45}$, R. Narayan ${ }^{11}$, D. I. Narrias Villar ${ }^{60 a}$, I. Naryshkin ${ }^{125}$, T. Naumann ${ }^{45}$, G. Navarro ${ }^{21}$, R. Nayyar ${ }^{7}$, H. A. Neal ${ }^{92}$, P. Yu. Nechaeva ${ }^{98}$, T. J. Neep ${ }^{138}$, A. Negri ${ }^{123 a, 123 b}$, M. Negrini ${ }^{22 a}, \quad$ S. Nektarijevic ${ }^{108}$,

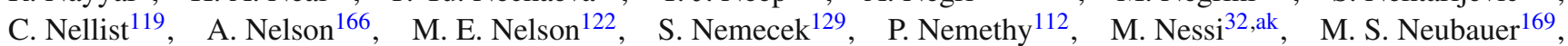
M. Neumann ${ }^{178}$, P. R. Newman ${ }^{19}$, T. Y. Ng ${ }^{62 c}$, T. Nguyen Manh ${ }^{97}$, R. B. Nickerson ${ }^{122}$, R. Nicolaidou ${ }^{138}$, J. Nielsen ${ }^{139}$, V. Nikolaenko ${ }^{132, \text { aj, I. Nikolic-Audit }}{ }^{83}$, K. Nikolopoulos ${ }^{19}$, J. K. Nilsen ${ }^{121}$, P. Nilsson ${ }^{27}$, Y. Ninomiya ${ }^{157}$, A. Nisati ${ }^{134 a}$, N. Nishu ${ }^{35 c}$, R. Nisius ${ }^{103}$, I. Nitsche ${ }^{46}$, T. Nobe ${ }^{157}$, Y. Noguchi ${ }^{71}$, M. Nomachi ${ }^{120}$, I. Nomidis ${ }^{31}$, M. A. Nomura ${ }^{27}$, T. Nooney ${ }^{79}$, M. Nordberg ${ }^{32}$, N. Norjoharuddeen ${ }^{122}$, O. Novgorodova ${ }^{47}$, S. Nowak ${ }^{103}$, M. Nozaki ${ }^{69}$, L. Nozka ${ }^{117}$, K. Ntekas ${ }^{166}$, E. Nurse ${ }^{81}$, F. Nuti ${ }^{91}$, K. O’ connor $^{25}$, D. C. O’Neil ${ }^{144}$, A. A. O’Rourke ${ }^{45}$, V. O’Shea ${ }^{56}$, F. G. Oakham ${ }^{31 d}$, H. Oberlack ${ }^{103}$, T. Obermann ${ }^{23}$, J. Ocariz ${ }^{83}$, A. Ochi ${ }^{70}$, I. Ochoa ${ }^{38}$, J. P. Ochoa-Ricoux ${ }^{34 a}$, S. Oda ${ }^{73}$, S. Odaka ${ }^{69}$, H. Ogren ${ }^{64}$, A. $\mathrm{Oh}^{87}$, S. H. Oh ${ }^{48}$, C. C. $\mathrm{Ohm}^{16}$, H. Ohman ${ }^{168}$, H. Oide ${ }^{53 a, 53 b}$, H. Okawa ${ }^{164}$, Y. Okumura ${ }^{157}$, T. Okuyama ${ }^{69}$, A. Olariu ${ }^{28 b}$ ， L. F. Oleiro Seabra ${ }^{128 a}$ ，S. A. Olivares Pino ${ }^{49}$ ， D. Oliveira Damazio ${ }^{27}$ ，A. Olszewski ${ }^{42}$, J. Olszowska ${ }^{42}$, A. Onofre ${ }^{128 a}$,128e, K. Onogi ${ }^{105}$, P. U. E. Onyisi ${ }^{11 y}$, H. Oppen ${ }^{121}$, M. J. Oreglia ${ }^{33}$, Y. Oren ${ }^{155}$, D. Orestano ${ }^{136 a, 136 b}$, 
N. Orlando ${ }^{62 b}$ ， R. S. Orr ${ }^{161}$ ， B. Osculati ${ }^{53 a, 53 b, *}$, R. Ospanov ${ }^{36 a}$ ， G. Otero y Garzon ${ }^{29}$, H. Otono ${ }^{73}$ ， M. Ouchrif ${ }^{137 d}$, F. Ould-Saada ${ }^{121}$, A. Ouraou ${ }^{138}$, K. P. Oussoren ${ }^{109}$, Q. Ouyang ${ }^{35 a}$, M. Owen ${ }^{56}$, R. E. Owen ${ }^{19}$, V. E. Ozcan ${ }^{20 a}$, N. Ozturk ${ }^{8}$, K. Pachal ${ }^{144}$, A. Pacheco Pages ${ }^{13}$ ， L. Pacheco Rodriguez ${ }^{138}$, C. Padilla Aranda ${ }^{13}$, S. Pagan Griso ${ }^{16}$, M. Paganini ${ }^{179}$, F. Paige ${ }^{27}$, G. Palacino ${ }^{64}$, S. Palazzo ${ }^{40 a, 40 b}$, S. Palestini3 ${ }^{32}$, M. Palka ${ }^{41 b}$, D. Pallin ${ }^{37}$, E. St. Panagiotopoulou ${ }^{10}$, I. Panagoulias ${ }^{10}$, C. E. Pandini ${ }^{83}$, J. G. Panduro Vazquez ${ }^{80}$, P. Pani ${ }^{32}$, S. Panitkin ${ }^{27}$, D. Pantea ${ }^{28 b}$, L. Paolozzi ${ }^{52}$, Th. D. Papadopoulou ${ }^{10}$, K. Papageorgiou ${ }^{9}$, A. Paramonov $^{6}$, D. Paredes Hernandez ${ }^{179}$, A. J. Parker ${ }^{75}$, M. A. Parker ${ }^{30}$, K. A. Parker ${ }^{45}$, F. Parodi ${ }^{53 a, 53 b}$, J. A. Parsons ${ }^{38}$, U. Parzefall ${ }^{51}$, V. R. Pascuzzi ${ }^{161}$, J. M. Pasner ${ }^{139}$, E. Pasqualucci ${ }^{134 a}$, S. Passaggio ${ }^{53 a}$, Fr. Pastore ${ }^{80}$, S. Pataraia ${ }^{178}$, J. R. Pater ${ }^{87}$, T. Pauly ${ }^{32}$, B. Pearson ${ }^{103}$, S. Pedraza Lopez ${ }^{170}$, R. Pedro ${ }^{128 a, 128 b}$, S. V. Peleganchuk ${ }^{111 c}$, O. Penc ${ }^{129}$, C. Peng ${ }^{35 a}, \quad$ H. Peng $^{36 a}$, J. Penwell ${ }^{64}$, B. S. Peralva ${ }^{26 b}, \quad$ M. M. Perego ${ }^{138}$, D. V. Perepelitsa ${ }^{27}$, L. Perini ${ }^{94 a}, 94 b$, H. Pernegger ${ }^{32}$, S. Perrella ${ }^{106 a, 106 b}$, R. Peschke ${ }^{45}$, V. D. Peshekhonov ${ }^{68, *}$, K. Peters ${ }^{45}$, R. F. Y. Peters ${ }^{87}$, B. A. Petersen ${ }^{32}$, T. C. Petersen ${ }^{39}$, E. Petit ${ }^{58}$, A. Petridis ${ }^{1}$, C. Petridou ${ }^{156}$, P. Petroff ${ }^{119}$, E. Petrolo ${ }^{134 a}$, M. Petrov ${ }^{122}$, F. Petrucci 136a,136b, N. E. Pettersson ${ }^{89}$, A. Peyaud ${ }^{138}$, R. Pezoa ${ }^{34 b}$, F. H. Phillips ${ }^{93}$, P. W. Phillips ${ }^{133}$,

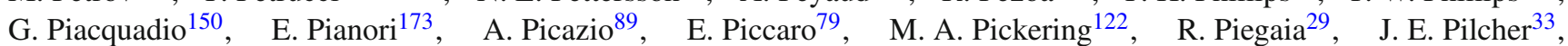
A. D. Pilkington ${ }^{87}$, A. W. J. Pin ${ }^{87}$, M. Pinamonti ${ }^{135 a, 135 b}$, J. L. Pinfold ${ }^{3}$, H. Pirumov ${ }^{45}$, M. Pitt ${ }^{175}$, L. Plazak ${ }^{146 a}$, M.-A. Pleier ${ }^{27}$, V. Pleskot ${ }^{86}$, E. Plotnikova ${ }^{68}$, D. Pluth ${ }^{67}$, P. Podberezko ${ }^{111}$, R. Poettgen ${ }^{148 a, 148 b}$, R. Poggi ${ }^{123 a, 123 b}$, L. Poggioli1 ${ }^{119}$, D. Pohl ${ }^{23}$, G. Polesello ${ }^{123 a}$, A. Poley ${ }^{45}$, A. Policicchio ${ }^{40,40 b}$, R. Polifka ${ }^{32}$, A. Polini ${ }^{22 a}$, C. S. Pollard ${ }^{56}$,

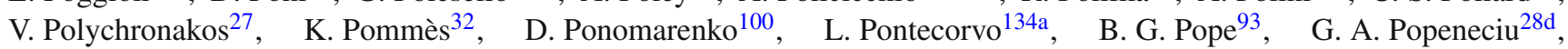
A. Poppleton ${ }^{32}$, S. Pospisil ${ }^{130}$, K. Potamianos ${ }^{16}$, I. N. Potrap ${ }^{68}$, C. J. Potter ${ }^{30}$, G. Poulard ${ }^{32}$, T. Poulsen ${ }^{84}$, J. Poveda ${ }^{32}$,

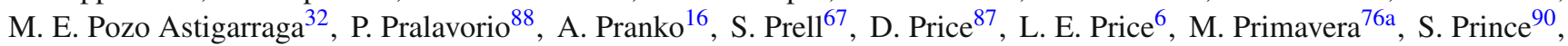
N. Proklova ${ }^{100}$, K. Prokofiev ${ }^{62 c}$, F. Prokoshin ${ }^{34 b}$, S. Protopopescu ${ }^{27}$, J. Proudfoot ${ }^{6}$, M. Przybycien ${ }^{41 a}$, A. Puri ${ }^{169}$, P. Puzo ${ }^{119}$, J. Qian ${ }^{92}$, G. Qin ${ }^{56}$, Y. Qin ${ }^{87}$, A. Quadt ${ }^{57}$, M. Queitsch-Maitland ${ }^{45}$, D. Quilty ${ }^{56}$, S. Raddum ${ }^{121}$, V. Radeka ${ }^{27}$, V. Radescu ${ }^{122}$, S. K. Radhakrishnan ${ }^{150}$, P. Radloff ${ }^{118}$, P. Rados ${ }^{91}$ ， F. Ragusa ${ }^{94 a, 94 b}$ ， G. Rahal ${ }^{182}$, J. A. Raine ${ }^{87}$, S. Rajagopalan ${ }^{27}$, C. Rangel-Smith ${ }^{168}$, T. Rashid ${ }^{119}$, S. Raspopov ${ }^{5}$, M. G. Ratti94a,94b, D. M. Rauch ${ }^{45}$, F. Rauscher ${ }^{102}$, S. Rave ${ }^{86}$, I. Ravinovich ${ }^{175}$ ， J. H. Rawling87, M. Raymond ${ }^{32}$ ， A. L. Read ${ }^{121}$, N. P. Readioff ${ }^{58,}$ M. Reale ${ }^{76 a, 76 b}$, D. M. Rebuzzi ${ }^{123 a}$,123b, A. Redelbach ${ }^{177}$, G. Redlinger ${ }^{27}$, R. Reece ${ }^{139}$ ，R. G. Reed ${ }^{147 c}$, K. Reeves ${ }^{44}$, L. Rehnisch ${ }^{17}$, J. Reichert ${ }^{124}$, A. Reiss ${ }^{86}$, C. Rembser ${ }^{32}$, H. Ren ${ }^{35 a}$, M. Rescigno ${ }^{134 a}$, S. Resconi ${ }^{94 a}$, E. D. Resseguie ${ }^{124}$, S. Rettie ${ }^{171}$, E. Reynolds ${ }^{19}$, O. L. Rezanova ${ }^{111 c}$, P. Reznicek ${ }^{131}$, R. Rezvani ${ }^{97}$, R. Richter ${ }^{103}$, S. Richter ${ }^{81}$, E. Richter-Was ${ }^{41 b}$, O. Ricken ${ }^{23}$, M. Ridel ${ }^{83}$, P. Rieck ${ }^{103}$, C. J. Riegel ${ }^{178}$, J. Rieger ${ }^{57}$, O. Rifki ${ }^{115}$, M. Rijssenbeek ${ }^{150}$, A. Rimoldi ${ }^{123 a, 123 b}$, M. Rimoldi ${ }^{18}$, L. Rinaldi ${ }^{22 a}$, G. Ripellino ${ }^{149}$, B. Ristic ${ }^{32}$, E. Ritsch ${ }^{32}$, I. Riu ${ }^{13}$, F. Rizatdinova ${ }^{116}$, E. Rizvi ${ }^{79}$, C. Rizzi ${ }^{13}$, R. T. Roberts ${ }^{87}$, S. H. Robertson ${ }^{900}$, A. Robichaud-Veronneau ${ }^{90}$, D. Robinson ${ }^{30}$, J. E. M. Robinson ${ }^{45}$, A. Robson ${ }^{56}$, E. Rocco ${ }^{86}$, C. Roda ${ }^{126 a, 126 b}$, Y. Rodina ${ }^{88, a l}$, S. Rodriguez Bosca ${ }^{170}$, A. Rodriguez Perez ${ }^{13}$, D. Rodriguez Rodriguez ${ }^{170}$, S. Roe ${ }^{32}$, C. S. Rogan ${ }^{59}$, O. Røhne ${ }^{121}$, J. Roloff ${ }^{59}$, A. Romaniouk ${ }^{100}$, M. Romano ${ }^{22 a, 22 b}$, S. M. Romano Saez ${ }^{37}$, E. Romero Adam ${ }^{170}$, N. Rompotis ${ }^{77}$, M. Ronzani ${ }^{51}$, L. Roos $^{83}$, S. Rosati ${ }^{134 a}$, K. Rosbach ${ }^{51}$, P. Rose ${ }^{139}$, N.-A. Rosien ${ }^{57}$, E. Rossi ${ }^{106 a, 106 b}$ ， L. P. Rossi ${ }^{53 a}$ ， J. H. N. Rosten ${ }^{30}$, R. Rosten ${ }^{140}, \quad$ M. Rotaru ${ }^{28 b}$ ， I. Roth ${ }^{175}$, J. Rothberg ${ }^{140}$, D. Rousseau ${ }^{119}$, A. Rozanov ${ }^{88}$, Y. Rozen ${ }^{154}$, X. Ruan ${ }^{147 c}$, F. Rubbo ${ }^{145}$, F. Rühr ${ }^{51}$, A. Ruiz-Martinez ${ }^{31}$, Z. Rurikova ${ }^{51}$, N. A. Rusakovich ${ }^{68}$, H. L. Russell ${ }^{90}$, J. P. Rutherfoord ${ }^{7}$, N. Ruthmann ${ }^{32}$, Y. F. Ryabov ${ }^{125}$, M. Rybar ${ }^{169}$, G. Rybkin ${ }^{119}$, S. Ryu ${ }^{6}$, A. Ryzhov ${ }^{132}$, G. F. Rzehorz ${ }^{57}$, A. F. Saavedra ${ }^{152}$, G. Sabato ${ }^{109}$, S. Sacerdoti ${ }^{29}$, H.F.-W. Sadrozinski ${ }^{139}$, R. Sadykov ${ }^{68}$, F. Safai Tehranii ${ }^{134 a}$, P. Saha ${ }^{110}$, M. Sahinsoy ${ }^{60 a}$, M. Saimpert ${ }^{45}$, M. Saito ${ }^{157}$, T. Saito ${ }^{157}$, H. Sakamoto ${ }^{157}$, Y. Sakurai ${ }^{174}$, G. Salamanna ${ }^{136 a, 136 b}$, J. E. Salazar Loyola ${ }^{34 b}$ ， D. Salek ${ }^{109}$ ， P. H. Sales De Bruin ${ }^{168}$, D. Salihagic ${ }^{103}$, A. Salnikov ${ }^{145}$, J. Salt ${ }^{170}$, D. Salvatore ${ }^{40 a, 40 b}$, F. Salvatore ${ }^{151}$, A. Salvucci ${ }^{62 a, 62 b, 62 c}$, A. Salzburger ${ }^{32}$, D. Sammel ${ }^{51}$, D. Sampsonidis ${ }^{156}$, D. Sampsonidou ${ }^{156}$, J. Sánchez ${ }^{170}$, V. Sanchez Martinez ${ }^{170}$, A. Sanchez Pineda ${ }^{167 a, 167 c}$, H. Sandaker $^{121}$, R. L. Sandbach ${ }^{79}$, C. O. Sander ${ }^{45}$, M. Sandhoff ${ }^{178}$, C. Sandoval ${ }^{21}$, D. P. C. Sankey ${ }^{133}$, M. Sannino ${ }^{53 a, 53 b}$, A. Sansoni ${ }^{50}$,

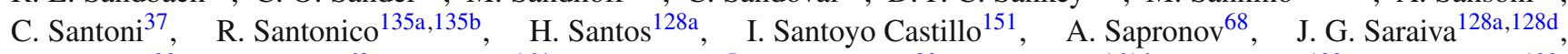

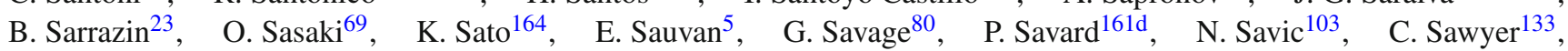
L. Sawyer ${ }^{82 t}$, J. Saxon ${ }^{33}$, C. Sbarra ${ }^{22 a}$, A. Sbrizzi ${ }^{22 a, 22 b}$, T. Scanlon ${ }^{81}, \quad$ D. A. Scannicchio ${ }^{166}, \quad$ M. Scarcella ${ }^{152}$, V. Scarfone ${ }^{40 a}$,40b , J. Schaarschmidt ${ }^{140}$, P. Schacht ${ }^{103}$, B. M. Schachtner ${ }^{102}$, D. Schaefer ${ }^{32}$, L. Schaefer ${ }^{124}$, R. Schaefer ${ }^{45}$, J. Schaeffer ${ }^{86}$, S. Schaepe ${ }^{23}$, S. Schaetzel ${ }^{60 b}$, U. Schäfer ${ }^{86}$, A. C. Schaffer ${ }^{119}$, D. Schaile ${ }^{102}$, R. D. Schamberger ${ }^{150}$, V. Scharf ${ }^{60 a}$, V. A. Schegelsky ${ }^{125}$, D. Scheirich ${ }^{131}$, M. Schernau ${ }^{166}$, C. Schiavi ${ }^{53 a, 53 b}$, S. Schier ${ }^{139}$, L. K. Schildgen ${ }^{23}$,

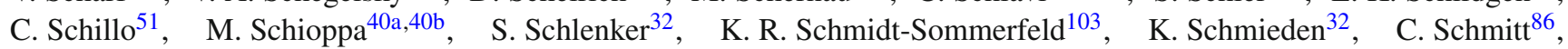
S. Schmitt ${ }^{45}$, S. Schmitz ${ }^{86}$, U. Schnoor ${ }^{51}$, L. Schoeffel ${ }^{138}$, A. Schoening ${ }^{60 b}$, B. D. Schoenrock ${ }^{93}$, E. Schopf ${ }^{23}$, M. Schott ${ }^{86}$, J. F. P. Schouwenberg ${ }^{108}$, J. Schovancova ${ }^{181}$, S. Schramm ${ }^{52}$, N. Schuh ${ }^{86}$, A. Schulte ${ }^{86}$, M. J. Schultens ${ }^{23}$, H.-C. Schultz-Coulon ${ }^{60 a}$, H. Schulz ${ }^{17}$, M. Schumacher ${ }^{51}$, B. A. Schumm ${ }^{139}$, Ph. Schune ${ }^{138}, \quad$ A. Schwartzman ${ }^{145}$,

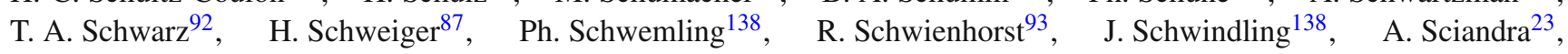




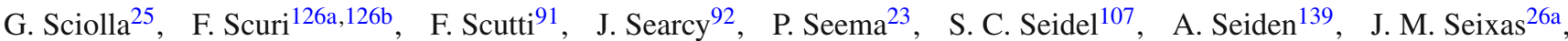
G. Sekhniaidze ${ }^{106 a}$ ， K. Sekhon ${ }^{92}$ ， S. J. Sekula ${ }^{43}$ ， N. Semprini-Cesari ${ }^{22 a, 22 b}$ ，S. Senkin ${ }^{37} ，$ C. Serfon ${ }^{121}$ ， L. Serin ${ }^{119}$, L. Serkin ${ }^{167 a, 167 b}$, M. Sessa ${ }^{136 a, 136 b}$, R. Seuster ${ }^{172}$, H. Severini ${ }^{115}$, T. Sfiligoj ${ }^{78}$, F. Sforza ${ }^{32}$, A. Sfyrla ${ }^{52}$, E. Shabalina ${ }^{57}$, N. W. Shaikh ${ }^{148 a, 148 b}$ ， L. Y. Shan ${ }^{35 a}$ ， R. Shang ${ }^{169}$ ， J. T. Shank ${ }^{24}$ ， M. Shapiro ${ }^{16}$ ， P. B. Shatalov ${ }^{99}$ ， K. Shaw ${ }^{167 a, 167 b,}$ S. M. Shaw ${ }^{87}$, A. Shcherbakova ${ }^{148 a, 148 b}$, C. Y. Shehu ${ }^{151}$, Y. Shen ${ }^{115}$ ， N. Sherafati ${ }^{31}$, P. Sherwood ${ }^{81}$, L. Shi ${ }^{153, a m}$,

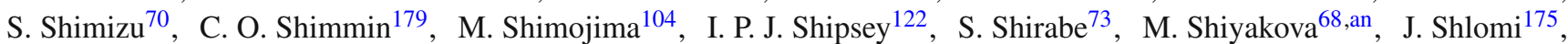
A. Shmeleva ${ }^{98}$, D. Shoaleh Saadi ${ }^{97}, \quad$ M. J. Shochet ${ }^{33}$, S. Shojaiii ${ }^{94 a}$, D. R. Shope ${ }^{115}$, S. Shrestha ${ }^{113}$, E. Shulga ${ }^{100}$, M. A. Shupe ${ }^{7}$, P. Sicho ${ }^{129}$, A. M. Sickles ${ }^{169}$, P. E. Sidebo ${ }^{149}$, E. Sideras Haddad ${ }^{147 c}$, O. Sidiropoulou ${ }^{177}$, A. Sidoti ${ }^{22 a, 22 b}$, F. Siegert ${ }^{47}$, Dj. Sijacki ${ }^{14}$ ，J. Silva ${ }^{128 a, 128 d}$, S. B. Silverstein ${ }^{148 a}$, V. Simak ${ }^{130}$, Lj. Simic ${ }^{14}$, S. Simion ${ }^{119}$, E. Simioni ${ }^{86}$, B. Simmons ${ }^{81}$, M. Simon ${ }^{86}$, P. Sinervo ${ }^{161}$, N. B. Sinev ${ }^{118}$, M. Sioli ${ }^{22 a, 22 b}$, G. Siragusa ${ }^{177}$, I. Siral ${ }^{92}$, S. Yu. Sivoklokov ${ }^{101}$, J. Sjölin ${ }^{148 a}, 148 b$, M. B. Skinner ${ }^{75}$, P. Skubic ${ }^{115}$, M. Slater ${ }^{19}$, T. Slavicek ${ }^{130}$, M. Slawinska ${ }^{42}$, K. Sliwa ${ }^{165}$, R. Slovak ${ }^{131}$, V. Smakhtin ${ }^{175}$, B. H. Smart ${ }^{5}$, J. Smiesko ${ }^{146 a}$, N. Smirnov ${ }^{100}$, S. Yu. Smirnov ${ }^{100}$, Y. Smirnov ${ }^{100}$, L. N. Smirnova ${ }^{101, a o}$, O. Smirnova ${ }^{84}$ ， J. W. Smith ${ }^{57}$ ， M. N. K. Smith ${ }^{38 ， R . ~ W . ~ S m i t h ~}{ }^{38}$ ，M. Smizanska ${ }^{75}$ ，K. Smolek ${ }^{130 \text {, A. A. Snesarev }}{ }^{98}$, I. M. Snyder ${ }^{118}$, S. Snyder ${ }^{27}$, R. Sobie ${ }^{1720}$, F. Socher ${ }^{47}$, A. Soffer ${ }^{155}$, A. Søgaard ${ }^{49}$, D. A. Soh ${ }^{153}$, G. Sokhrannyi ${ }^{78}$,

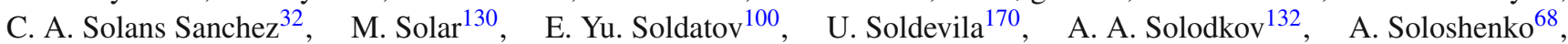
O. V. Solovyanov 132 , V. Solovyev ${ }^{125}$, P. Sommer ${ }^{51}$, H. Son ${ }^{165}$, A. Sopczak ${ }^{130}$, D. Sosa ${ }^{60 b}$, C. L. Sotiropoulou ${ }^{126 a, 126 b}$, R. Soualah ${ }^{167 a, 167 c}$, A. M. Soukharev ${ }^{111}$, D. South ${ }^{45}$, B. C. Sowden ${ }^{80}, \quad$ S. Spagnolo ${ }^{76 a, 76 b}, \quad$ M. Spalla ${ }^{126 a, 126 b}$, M. Spangenberg ${ }^{173}$, F. Spanò ${ }^{80}$, D. Sperlich ${ }^{17}$, F. Spettel ${ }^{103}$, T. M. Spieker ${ }^{60}$, R. Spighi ${ }^{22 a}$, G. Spigo ${ }^{32}$, L. A. Spiller ${ }^{91}$, M. Spousta ${ }^{131}$, R. D. St. Denis ${ }^{56, *}$, A. Stabile ${ }^{94 a}$, R. Stamen ${ }^{60 a}$, S. Stamm ${ }^{17}$, E. Stanecka ${ }^{42}$, R. W. Stanek ${ }^{6}$, C. Stanescu ${ }^{136 a}$, M. M. Stanitzki ${ }^{45}$, B. S. Stapf ${ }^{109}$, S. Stapnes ${ }^{121}$, E. A. Starchenko ${ }^{132}$, G. H. Stark ${ }^{33}$, J. Stark ${ }^{58}$, S. H Stark ${ }^{39}$, P. Staroba ${ }^{129}$, P. Starovoitov ${ }^{60 a}$ ， S. Stärz ${ }^{32}$ ， R. Staszewski ${ }^{42}$, P. Steinberg ${ }^{27}$ ， B. Stelzer ${ }^{144}$ ， H. J. Stelzer ${ }^{32}$, O. Stelzer-Chilton ${ }^{163 a}$, H. Stenzel ${ }^{55}$, G. A. Stewart ${ }^{56}$, M. C. Stockton ${ }^{118}$, M. Stoebe ${ }^{90}$, G. Stoicea ${ }^{28 b}$, P. Stolte ${ }^{57}$, S. Stonjek ${ }^{103}$, A. R. Stradling ${ }^{8}$, A. Straessner ${ }^{47}$, M. E. Stramaglia ${ }^{18}$, J. Strandberg ${ }^{149}$, S. Strandberg ${ }^{148 a, 148 b}$, M. Strauss ${ }^{115}$, P. Strizenec ${ }^{146 b}$, R. Ströhmer ${ }^{177}$, D. M. Strom ${ }^{118}$, R. Stroynowski ${ }^{43}$, A. Strubig ${ }^{108}$, S. A. Stucci ${ }^{27}$, B. Stugu ${ }^{15}$, N. A. Styles ${ }^{45}$, D. Su ${ }^{145}, \quad$ J. Su ${ }^{127}$, S. Suchek ${ }^{60 a}$, Y. Sugaya ${ }^{120}$, M. Suk ${ }^{130}$, V. V. Sulin ${ }^{98}$, DMS Sultan ${ }^{162 a, 162 b}$, S. Sultansoy ${ }^{4 c}$, T. Sumida ${ }^{71}$, S. Sun ${ }^{59}$, X. Sun ${ }^{3}$, K. Suruliz ${ }^{151}$, C. J. E. Suster ${ }^{152}$, M. R. Sutton ${ }^{151}$, S. Suzuki ${ }^{69}$, M. Svatos ${ }^{129}$, M. Swiatlowski ${ }^{33}$, S. P. Swift ${ }^{2}$, I. Sykora ${ }^{146 a}$, T. Sykora ${ }^{131}$, D. Ta ${ }^{51}$, K. Tackmann ${ }^{45}$, J. Taenzer ${ }^{155}$, A. Taffard ${ }^{166}$, R. Tafirout ${ }^{163 a}$, E. Tahirovic ${ }^{79}$, N. Taiblum ${ }^{155}$, H. Takai ${ }^{27}$, R. Takashima ${ }^{72}$, E. H. Takasugi ${ }^{103}$, T. Takeshita ${ }^{142}$, Y. Takubo ${ }^{69}$, M. Talby ${ }^{88}$, A. A. Talyshev ${ }^{111 \text { c }}$, J. Tanaka ${ }^{157}$, M. Tanaka ${ }^{159}$, R. Tanaka ${ }^{119}$, S. Tanaka ${ }^{69}$, R. Tanioka ${ }^{70}$, B. B. Tannenwald ${ }^{113}$, S. Tapia Araya ${ }^{34 b}$, S. Tapprogge ${ }^{86}$, S. Tarem ${ }^{154}$, G. F. Tartarelli ${ }^{94 a}$, P. Tas ${ }^{131}$, M. Tasevsky ${ }^{129}$, T. Tashiro ${ }^{71}$, E. Tassi4 ${ }^{40 a, 40 b}$, A. Tavares Delgado ${ }^{128 a, 128 b}$, Y. Tayalati $^{137 \mathrm{e}}$, A. C. Taylor ${ }^{107}$, G. N. Taylor ${ }^{91}$, P. T. E. Taylor ${ }^{11}$, W. Taylor ${ }^{163 \mathrm{~b}}$, P. Teixeira-Dias ${ }^{80}$, D. Temple ${ }^{144}$, H. Ten Kate ${ }^{32}$, P. K. Teng ${ }^{153}$, J. J. Teoh ${ }^{120}$, F. Tepel ${ }^{178}$, S. Terada ${ }^{69}$, K. Terashi ${ }^{157}$, J. Terron ${ }^{85}$, S. Terzo ${ }^{13}$, M. Testa ${ }^{50}$, R. J. Teuscher ${ }^{1610}$, T. Theveneaux-Pelzer ${ }^{88}$, J. P. Thomas ${ }^{19}$, J. Thomas-Wilsker ${ }^{80}$, P. D. Thompson ${ }^{19}$, A. S. Thompson ${ }^{56}$, L. A. Thomsen ${ }^{179}$, E. Thomson ${ }^{124}$, M. J. Tibbetts ${ }^{16}$, R. E. Ticse Torres ${ }^{88}$, V. O. Tikhomirov ${ }^{98, a p, ~ Y u . ~ A . ~ T i k h o n o v ~}{ }^{111 c}$, S. Timoshenko ${ }^{100}$, P. Tipton ${ }^{179}$, S. Tisserant ${ }^{88}$, K. Todome ${ }^{159}$, S. Todorova-Nova ${ }^{5}$, J. Tojo $^{73}$, S. Tokár $^{146 a}$, K. Tokushuku ${ }^{69}$, E. Tolley ${ }^{59}$, L. Tomlinson ${ }^{87}$, M. Tomoto ${ }^{105}$, L. Tompkins ${ }^{145}$,aq, K. Toms ${ }^{107}$, B. Tong ${ }^{59}$, P. Tornambe ${ }^{51}$, E. Torrence ${ }^{118}$, H. Torres ${ }^{144}$, E. Torró Pastor ${ }^{140}, \quad$ J. Toth ${ }^{88, \text { ar }}, \quad$ F. Touchard ${ }^{88}, \quad$ D. R. Tovey ${ }^{141}, \quad$ C. J. Treado ${ }^{112}, \quad$ T. Trefzger ${ }^{177}$, F. Tresoldi ${ }^{151}$, A. Tricoli ${ }^{27}$, I. M. Trigger ${ }^{163 a}$, S. Trincaz-Duvoid ${ }^{83}$, M. F. Tripiana ${ }^{13}$, W. Trischuk ${ }^{161}$, B. Trocmé ${ }^{58}$, A. Trofymov ${ }^{45}, \quad$ C. Troncon ${ }^{94 a}, \quad$ M. Trottier-McDonald ${ }^{16}, \quad$ M. Trovatelli ${ }^{172}, \quad$ L. Truong ${ }^{167 a, 167 c}, \quad$ M. Trzebinski ${ }^{42}$, A. Trzupek ${ }^{42}$, K. W. Tsang ${ }^{62 a}$, J.C.-L. Tseng ${ }^{122}$, P. V. Tsiareshka ${ }^{95}$, G. Tsipolitis ${ }^{10}$, N. Tsirintanis ${ }^{9}$, S. Tsiskaridze ${ }^{13}$, V. Tsiskaridze ${ }^{51}$ ， E. G. Tskhadadze ${ }^{54 a}$ ， K. M. Tsui ${ }^{62 a}$ ， I. I. Tsukerman ${ }^{99}$ ， V. Tsulaia ${ }^{16}$ ， S. Tsuno ${ }^{69}$ ， D. Tsybychev ${ }^{150}$, Y. Tu ${ }^{62 b}$, A. Tudorache ${ }^{28 b}$, V. Tudorache ${ }^{28 b}$, T. T. Tulbure ${ }^{28 a}$, A. N. Tuna ${ }^{59}$, S. A. Tupputi ${ }^{22 a, 22 b}$, S. Turchikhin ${ }^{68}$, D. Turgeman ${ }^{175}$, I. Turk Cakir ${ }^{4 b, a s}$, R. Turra ${ }^{94 a}$, P. M. Tuts ${ }^{38}$, G. Ucchielli ${ }^{22 a, 22 b}$, I. Ueda ${ }^{69}$, M. Ughetto ${ }^{148 a, 148 b}$, F. Ukegawa ${ }^{164}$, G. Unal $^{32}$, A. Undrus ${ }^{27}$, G. Unel ${ }^{166}$, F. C. Ungaro ${ }^{91}$, Y. Unno ${ }^{69}$, C. Unverdorben ${ }^{102}$, J. Urban ${ }^{146 b}$,

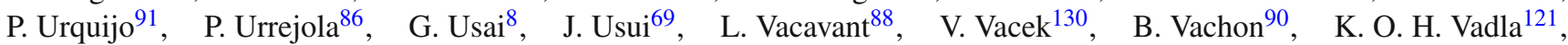
A. Vaidya ${ }^{81}$, C. Valderanis ${ }^{102}$, E. Valdes Santurio ${ }^{148 a, 148 b}$, M. Valente ${ }^{52}$, S. Valentinetti ${ }^{22 a, 22 b}$, A. Valero ${ }^{170}$, L. Valéry ${ }^{13}$, S. Valkar ${ }^{131}$ ， A. Vallier ${ }^{5}$ ， J. A. Valls Ferrer ${ }^{170}$, W. Van Den Wollenberg ${ }^{109}$ ， H. van der Graaf ${ }^{109}$, P. van Gemmeren ${ }^{6}$, J. Van Nieuwkoop ${ }^{144}$, I. van Vulpen ${ }^{109}$, M. C. van Woerden ${ }^{109}$ ， M. Vanadia ${ }^{135 a, 135 b}$, W. Vandelli ${ }^{32}$, A. Vaniachine ${ }^{160}$, P. Vankov ${ }^{109}$, G. Vardanyan ${ }^{180}$, R. Vari ${ }^{134 a}$, E. W. Varnes ${ }^{7}$, C. Varni ${ }^{53 a, 53 b}$, T. Varol ${ }^{43}$, D. Varouchas ${ }^{119}$, A. Vartapetian ${ }^{8}$, K. E. Varvel1 ${ }^{152}$, J. G. Vasquez ${ }^{179}$, G. A. Vasquez ${ }^{34 b}$, F. Vazeille ${ }^{37}$, T. Vazquez Schroeder ${ }^{90}$, J. Veatch ${ }^{57}$, V. Veeraraghavan ${ }^{7}$, L. M. Veloce ${ }^{161}$, F. Veloso ${ }^{128 a, 128 c}$, S. Veneziano ${ }^{134 a}$, A. Ventura ${ }^{76 a, 76 b}$, M. Venturi ${ }^{172}$, N. Venturi ${ }^{32}$, A. Venturini ${ }^{25}$, $\begin{array}{ll}\text { V. Vercesi } & 123 a\end{array}$ M. Verducci ${ }^{136 a, 136 b}$, W. Verkerke ${ }^{109}$, A. T. Vermeulen ${ }^{109}$, J. C. Vermeulen ${ }^{109}$, M. C. Vetterli ${ }^{144 d}$, N. Viaux Maira ${ }^{34 b}$ ， O. Viazlo ${ }^{84}$ ， I. Vichou ${ }^{169, *}, \quad$ T. Vickey ${ }^{141}, \quad$ O. E. Vickey Boeriu ${ }^{141}$ ， G. H. A. Viehhauser ${ }^{122}$, 


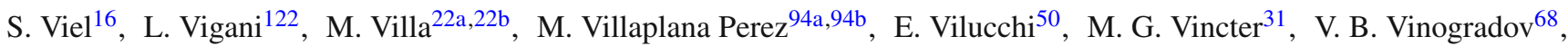
A. Vishwakarma ${ }^{45}$, C. Vittori ${ }^{22 a, 22 b}$, I. Vivarelli ${ }^{151}$, S. Vlachos ${ }^{10}$, M. Vlasak ${ }^{130}$, M. Vogel ${ }^{178}$, P. Vokac ${ }^{130}$, G. Volpi ${ }^{126 a, 126 b}$, H. von der Schmitt ${ }^{103}$ ， E. von Toerne ${ }^{23}$ ， V. Vorobel ${ }^{131}, \quad$ K. Vorobev ${ }^{100}, \quad$ M. Vos ${ }^{170}, \quad$ R. Voss ${ }^{32}$, J. H. Vossebeld ${ }^{77}$, N. Vranjes ${ }^{14}$, M. Vranjes Milosavljevic ${ }^{14}$, V. Vrba ${ }^{130}$, M. Vreeswijk ${ }^{109}$ ， R. Vuillermet ${ }^{32}$, I. Vukotic ${ }^{33}$, P. Wagner ${ }^{23}$, W. Wagner ${ }^{178}$, J. Wagner-Kuhr ${ }^{102}$, H. Wahlberg ${ }^{74}$, S. Wahrmund ${ }^{47}$, J. Wakabayashi ${ }^{105}$, J. Walder ${ }^{75}$, R. Walker ${ }^{102}$, W. Walkowiak ${ }^{143}$, V. Wallangen ${ }^{148 a, 148 b}$, C. Wang ${ }^{35 b}$, C. Wang ${ }^{36, a t}$, F. Wang ${ }^{176}$, H. Wang ${ }^{16}$, H. Wang ${ }^{3}$, J. Wang ${ }^{45}$, J. Wang ${ }^{152}$, Q. Wang ${ }^{115}$, R. Wang ${ }^{6}$, S. M. Wang ${ }^{153}$, T. Wang ${ }^{38}$, W. Wang ${ }^{153, \text { au }, \text { W. Wang }}{ }^{36 a}$, Z. Wang ${ }^{36 c}$, C. Wanotayaroj ${ }^{118}$, A. Warburton ${ }^{90}$, C. P. Ward $^{30}$, D. R. Wardrope ${ }^{81}$, A. Washbrook ${ }^{49}$, P. M. Watkins ${ }^{19}$, A. T. Watson ${ }^{19}$, M. F. Watson ${ }^{19}$, G. Watts ${ }^{140}$, S. Watts ${ }^{87}$, B. M. Waugh ${ }^{81}$, A. F. Webb ${ }^{11}$, S. Webb ${ }^{86}$, M. S. Weber ${ }^{18}$, S. W. Weber ${ }^{177}$, S. A. Weber ${ }^{31}$, J. S. Webster ${ }^{6}$, A. R. Weidberg ${ }^{122}$, B. Weinert ${ }^{64}$, J. Weingarten ${ }^{57}$, M. Weirich ${ }^{86}$, C. Weiser ${ }^{51}$, H. Weits ${ }^{109}$, P. S. Wells ${ }^{32}$, T. Wenaus ${ }^{27}$, T. Wengler ${ }^{32}$, S. Wenig ${ }^{32}$, N. Wermes ${ }^{23}$, M. D. Werner ${ }^{67}$, P. Werner ${ }^{32}$, M. Wessels ${ }^{60 a}$, T. D. Weston ${ }^{18}$, K. Whalen ${ }^{118}$, N. L. Whallon ${ }^{140}$, A. M. Wharton ${ }^{75}$, A. S. White ${ }^{92}$, A. White ${ }^{8}$, M. J. White ${ }^{1}$, R. White ${ }^{34 b}$, D. Whiteson ${ }^{166}$, B. W. Whitmore ${ }^{75}$, F. J. Wickens ${ }^{133}$, W. Wiedenmann ${ }^{176}$, M. Wielers ${ }^{133}$, C. Wiglesworth ${ }^{39}$, L. A. M. Wiik-Fuchs ${ }^{23}$, A. Wildauer ${ }^{103}$, F. Wilk ${ }^{87}$, H. G. Wilkens ${ }^{32}$, H. H. Williams ${ }^{124}$, S. Williams ${ }^{109}$, C. Willis ${ }^{93}$, S. Willocq ${ }^{89}$, J. A. Wilson ${ }^{19}$, I. Wingerter-Seez ${ }^{5}$, E. Winkels ${ }^{151}$, F. Winklmeier ${ }^{118}$, O. J. Winston ${ }^{151}$, B. T. Winter ${ }^{23}$, M. Wittgen ${ }^{145}$, M. Wobisch ${ }^{82 t}$, T. M. H. Wolf ${ }^{109}$, R. Wolff ${ }^{88}$, M. W. Wolter ${ }^{42}$, H. Wolters ${ }^{128 a, 128 c}$, V. W. S. Wong ${ }^{171}$, S. D. Worm ${ }^{19}$, B. K. Wosiek ${ }^{42}$, J. Wotschack ${ }^{32}$, K. W. Wozniak ${ }^{42}$, M. Wu ${ }^{33}$, S. L. Wu ${ }^{176}$, X. Wu ${ }^{52}$, Y. Wu ${ }^{92}$, T. R. Wyatt ${ }^{87}$, B. M. Wynne ${ }^{49}$, S. Xella ${ }^{39}$, Z. Xi ${ }^{92}$, L. Xia ${ }^{35 c}$, D. Xu ${ }^{35 a}$, L. Xu ${ }^{27}$, B. Yabsley ${ }^{152}$, S. Yacoob ${ }^{147 a}$, D. Yamaguchi ${ }^{159}$, Y. Yamaguchi ${ }^{120}$, A. Yamamoto ${ }^{69}$, S. Yamamoto ${ }^{157}$, T. Yamanaka ${ }^{157}$, M. Yamatani ${ }^{157}$, K. Yamauchi ${ }^{105}$, Y. Yamazaki ${ }^{70}$, Z. Yan ${ }^{24}$, H. Yang ${ }^{36 c}$, H. Yang ${ }^{16}$, Y. Yang ${ }^{153}$, Z. Yang ${ }^{15}$, W.-M. Yao ${ }^{16}$, Y. C. Yap ${ }^{83}$, Y. Yasu ${ }^{69}$, E. Yatsenko ${ }^{5}$, K. H. Yau Wong ${ }^{23}$, J. Ye ${ }^{43}$, S. Ye ${ }^{27}$, I. Yeletskikh ${ }^{68}$, E. Yigitbasi ${ }^{24}$, E. Yildirim ${ }^{86}$, K. Yorita ${ }^{174}$, K. Yoshihara ${ }^{124}$, C. Young ${ }^{145}$, C. J. S. Young ${ }^{32}$, J. Yu ${ }^{8}$, J. Yu ${ }^{67}$, S. P. Y. Yuen ${ }^{23}$, I. Yusuff ${ }^{30, a v}$, B. Zabinski ${ }^{42}$, G. Zacharis ${ }^{10}$, R. Zaidan ${ }^{13}$, A. M. Zaitsev ${ }^{132, a j}$, N. Zakharchuk ${ }^{45}$, J. Zalieckas ${ }^{15}$, A. Zaman ${ }^{150}$, S. Zambito ${ }^{59}$, D. Zanzi ${ }^{91}$, C. Zeitnitz ${ }^{178}$, G. Zemaityte ${ }^{122}$, A. Zemla ${ }^{41 a}$, J. C. Zeng ${ }^{169}$, Q. Zeng ${ }^{145}$, O. Zenin ${ }^{132}$, T. Ženiš ${ }^{146 a}$, D. Zerwas ${ }^{119}$, D. Zhang ${ }^{92}$, F. Zhang ${ }^{176}$, G. Zhang ${ }^{36 a, a w}$, H. Zhang ${ }^{35 b}$, J. Zhang ${ }^{6}$, L. Zhang ${ }^{51}$, L. Zhang ${ }^{36 a}$, M. Zhang ${ }^{169}$, P. Zhang ${ }^{35 b}$, R. Zhang ${ }^{23}$, R. Zhang ${ }^{36 a t}$, X. Zhang ${ }^{36 b}$, Y. Zhang ${ }^{35 a}$, Z. Zhang ${ }^{119}$, X. Zhao ${ }^{43}$, Y. Zhao ${ }^{36 b, a x}$, Z. Zhao ${ }^{36 a}$, A. Zhemchugov ${ }^{68}$, B. Zhou ${ }^{92}$, C. Zhou ${ }^{176}$, L. Zhou ${ }^{43}$, M. Zhou ${ }^{35 a}$, M. Zhou ${ }^{150}$, N. Zhou ${ }^{35 c}$, C. G. Zhu ${ }^{36 b}$, H. Zhu ${ }^{35 a}$, J. Zhu ${ }^{92}$, Y. Zhu ${ }^{36 a}$, X. Zhuang ${ }^{35 a}$, K. Zhukov98, A. Zibell ${ }^{177}$, D. Zieminska ${ }^{64}$, N. I. Zimine ${ }^{68}$, C. Zimmermann ${ }^{86}$, S. Zimmermann ${ }^{51}$, Z. Zinonos ${ }^{103}$, M. Zinser ${ }^{86}$, M. Ziolkowski ${ }^{143}$, L. Živković ${ }^{14}$, G. Zobernig ${ }^{176}$, A. Zoccoli ${ }^{22 a, 22 b}$, R. Zou ${ }^{33}$, M. zur Nedden ${ }^{17}$, L. Zwalinski ${ }^{32}$

${ }^{1}$ Department of Physics, University of Adelaide, Adelaide, Australia

${ }^{2}$ Physics Department, SUNY Albany, Albany, NY, USA

${ }^{3}$ Department of Physics, University of Alberta, Edmonton, AB, Canada

4 (a) Department of Physics, Ankara University, Ankara, Turkey; ${ }^{(b)}$ Istanbul Aydin University, Istanbul, Turkey; ${ }^{(c)}$ Division of Physics, TOBB University of Economics and Technology, Ankara, Turkey

${ }^{5}$ LAPP, CNRS/IN2P3 and Université Savoie Mont Blanc, Annecy-le-Vieux, France

${ }^{6}$ High Energy Physics Division, Argonne National Laboratory, Argonne, IL, USA

${ }^{7}$ Department of Physics, University of Arizona, Tucson, AZ, USA

${ }^{8}$ Department of Physics, The University of Texas at Arlington, Arlington, TX, USA

${ }^{9}$ Physics Department, National and Kapodistrian University of Athens, Athens, Greece

${ }^{10}$ Physics Department, National Technical University of Athens, Zografou, Greece

${ }^{11}$ Department of Physics, The University of Texas at Austin, Austin, TX, USA

${ }^{12}$ Institute of Physics, Azerbaijan Academy of Sciences, Baku, Azerbaijan

${ }^{13}$ Institut de Física d'Altes Energies (IFAE), The Barcelona Institute of Science and Technology, Barcelona, Spain

${ }^{14}$ Institute of Physics, University of Belgrade, Belgrade, Serbia

${ }^{15}$ Department for Physics and Technology, University of Bergen, Bergen, Norway

${ }^{16}$ Physics Division, Lawrence Berkeley National Laboratory and University of California, Berkeley, CA, USA

${ }^{17}$ Department of Physics, Humboldt University, Berlin, Germany

${ }^{18}$ Albert Einstein Center for Fundamental Physics and Laboratory for High Energy Physics, University of Bern, Bern, Switzerland

${ }^{19}$ School of Physics and Astronomy, University of Birmingham, Birmingham, UK 
20 (a) Department of Physics, Bogazici University, Istanbul, Turkey; ${ }^{(b)}$ Department of Physics Engineering, Gaziantep University, Gaziantep, Turkey; ${ }^{(c)}$ Faculty of Engineering and Natural Sciences, Istanbul Bilgi University, Istanbul, Turkey; ${ }^{(d)}$ Faculty of Engineering and Natural Sciences, Bahcesehir University, Istanbul, Turkey

${ }^{21}$ Centro de Investigaciones, Universidad Antonio Narino, Bogotá, Colombia

22 (a) INFN Sezione di Bologna, Bologna, Italy; ${ }^{(b)}$ Dipartimento di Fisica e Astronomia, Università di Bologna, Bologna, Italy

${ }^{23}$ Physikalisches Institut, University of Bonn, Bonn, Germany

24 Department of Physics, Boston University, Boston, MA, USA

25 Department of Physics, Brandeis University, Waltham, MA, USA

26 (a) Universidade Federal do Rio De Janeiro COPPE/EE/IF, Rio de Janeiro, Brazil; (b) Electrical Circuits Department, Federal University of Juiz de Fora (UFJF), Juiz de Fora, Brazil; ${ }^{(c)}$ Federal University of Sao Joao del Rei (UFSJ), Sao Joao del Rei, Brazil; ${ }^{(d)}$ Instituto de Fisica, Universidade de Sao Paulo, São Paulo, Brazil

27 Physics Department, Brookhaven National Laboratory, Upton, NY, USA

28 (a) Transilvania University of Brasov, Brasov, Romania; ${ }^{(b)}$ Horia Hulubei National Institute of Physics and Nuclear Engineering, Bucharest, Romania; ${ }^{(c)}$ Department of Physics, Alexandru Ioan Cuza University of Iasi, Iasi, Romania; (d) National Institute for Research and Development of Isotopic and Molecular Technologies, Physics Department, Cluj Napoca, Romania; ${ }^{(e)}$ University Politehnica Bucharest, Bucharest, Romania; ${ }^{(f)}$ West University in Timisoara, Timisoara, Romania

${ }^{29}$ Departamento de Física, Universidad de Buenos Aires, Buenos Aires, Argentina

${ }^{30}$ Cavendish Laboratory, University of Cambridge, Cambridge, UK

31 Department of Physics, Carleton University, Ottawa, ON, Canada

32 CERN, Geneva, Switzerland

${ }^{33}$ Enrico Fermi Institute, University of Chicago, Chicago, IL, USA

34 (a) Departamento de Física, Pontificia Universidad Católica de Chile, Santiago, Chile; (b) Departamento de Física, Universidad Técnica Federico Santa María, Valparaiso, Chile

35 (a) Institute of High Energy Physics, Chinese Academy of Sciences, Beijing, China; ${ }^{(b)}$ Department of Physics, Nanjing University, Nanjing, Jiangsu, China; ${ }^{(c)}$ Physics Department, Tsinghua University, Beijing 100084, China

36 (a) Department of Modern Physics and State Key Laboratory of Particle Detection and Electronics, University of Science and Technology of China, Hefei, Anhui, China; ${ }^{(b)}$ School of Physics, Shandong University, Jinan, Shandong, China; ${ }^{(c)}$ Department of Physics and Astronomy, Key Laboratory for Particle Physics, Astrophysics and Cosmology, Ministry of Education; Shanghai Key Laboratory for Particle Physics and Cosmology, Shanghai Jiao Tong University, Shanghai (also at PKU-CHEP), Shanghai, China

${ }^{37}$ Université Clermont Auvergne, CNRS/IN2P3, LPC, Clermont-Ferrand, France

38 Nevis Laboratory, Columbia University, Irvington, NY, USA

${ }^{39}$ Niels Bohr Institute, University of Copenhagen, Kobenhavn, Denmark

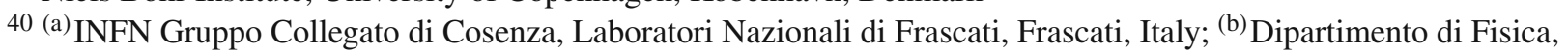
Università della Calabria, Rende, Italy

41 (a) Faculty of Physics and Applied Computer Science, AGH University of Science and Technology, Krakow,

Poland; (b) Marian Smoluchowski Institute of Physics, Jagiellonian University, Krakow, Poland

42 Institute of Nuclear Physics, Polish Academy of Sciences, Krakow, Poland

43 Physics Department, Southern Methodist University, Dallas, TX, USA

44 Physics Department, University of Texas at Dallas, Richardson, TX, USA

45 DESY, Hamburg and Zeuthen, Germany

${ }^{46}$ Lehrstuhl für Experimentelle Physik IV, Technische Universität Dortmund, Dortmund, Germany

${ }^{47}$ Institut für Kern- und Teilchenphysik, Technische Universität Dresden, Dresden, Germany

48 Department of Physics, Duke University, Durham, NC, USA

${ }^{49}$ SUPA-School of Physics and Astronomy, University of Edinburgh, Edinburgh, UK

${ }^{50}$ INFN Laboratori Nazionali di Frascati, Frascati, Italy

${ }^{51}$ Fakultät für Mathematik und Physik, Albert-Ludwigs-Universität, Freiburg, Germany

52 Departement de Physique Nucleaire et Corpusculaire, Université de Genève, Geneva, Switzerland

53 (a) INFN Sezione di Genova, Genoa, Italy; ${ }^{(b)}$ Dipartimento di Fisica, Università di Genova, Genova, Italy

54 (a) E. Andronikashvili Institute of Physics, Iv. Javakhishvili Tbilisi State University, Tbilisi, Georgia; ${ }^{(b)}$ High Energy

Physics Institute, Tbilisi State University, Tbilisi, Georgia 
55 II Physikalisches Institut, Justus-Liebig-Universität Giessen, Giessen, Germany

${ }^{56}$ SUPA-School of Physics and Astronomy, University of Glasgow, Glasgow, UK

${ }^{57}$ II Physikalisches Institut, Georg-August-Universität, Göttingen, Germany

${ }^{58}$ Laboratoire de Physique Subatomique et de Cosmologie, Université Grenoble-Alpes, CNRS/IN2P3, Grenoble, France

${ }^{59}$ Laboratory for Particle Physics and Cosmology, Harvard University, Cambridge, MA, USA

60 (a) Kirchhoff-Institut für Physik, Ruprecht-Karls-Universität Heidelberg, Heidelberg, Germany; ${ }^{(b)}$ Physikalisches Institut,

Ruprecht-Karls-Universität Heidelberg, Heidelberg, Germany; ${ }^{(c)}$ ZITI Institut für technische Informatik,

Ruprecht-Karls-Universität Heidelberg, Mannheim, Germany

${ }^{61}$ Faculty of Applied Information Science, Hiroshima Institute of Technology, Hiroshima, Japan

62 (a) Department of Physics, The Chinese University of Hong Kong, Shatin, N.T., Hong Kong; ${ }^{(b)}$ Department of Physics,

The University of Hong Kong, Hong Kong, China; ${ }^{(c)}$ Department of Physics and Institute for Advanced Study, The Hong

Kong University of Science and Technology, Clear Water Bay, Kowloon, Hong Kong, China

${ }^{63}$ Department of Physics, National Tsing Hua University, Hsinchu, Taiwan

${ }^{64}$ Department of Physics, Indiana University, Bloomington, IN, USA

${ }^{65}$ Institut für Astro- und Teilchenphysik, Leopold-Franzens-Universität, Innsbruck, Austria

${ }^{66}$ University of Iowa, Iowa City, IA, USA

${ }^{67}$ Department of Physics and Astronomy, Iowa State University, Ames, IA, USA

68 Joint Institute for Nuclear Research, JINR Dubna, Dubna, Russia

${ }^{69}$ KEK, High Energy Accelerator Research Organization, Tsukuba, Japan

${ }^{70}$ Graduate School of Science, Kobe University, Kobe, Japan

${ }^{71}$ Faculty of Science, Kyoto University, Kyoto, Japan

${ }^{72}$ Kyoto University of Education, Kyoto, Japan

${ }^{73}$ Research Center for Advanced Particle Physics and Department of Physics, Kyushu University, Fukuoka, Japan

${ }^{74}$ Instituto de Física La Plata, Universidad Nacional de La Plata and CONICET, La Plata, Argentina

75 Physics Department, Lancaster University, Lancaster, UK

76 (a) INFN Sezione di Lecce, Lecce, Italy; ${ }^{\left({ }^{b}\right)}$ Dipartimento di Matematica e Fisica, Università del Salento, Lecce, Italy

77 Oliver Lodge Laboratory, University of Liverpool, Liverpool, UK

${ }^{78}$ Department of Experimental Particle Physics, Jožef Stefan Institute and Department of Physics, University of Ljubljana, Ljubljana, Slovenia

${ }^{79}$ School of Physics and Astronomy, Queen Mary University of London, London, UK

${ }^{80}$ Department of Physics, Royal Holloway University of London, Surrey, UK

${ }^{81}$ Department of Physics and Astronomy, University College London, London, UK

${ }^{82}$ Louisiana Tech University, Ruston, LA, USA

${ }^{83}$ Laboratoire de Physique Nucléaire et de Hautes Energies, UPMC and Université Paris-Diderot and CNRS/IN2P3, Paris, France

${ }^{84}$ Fysiska institutionen, Lunds universitet, Lund, Sweden

${ }^{85}$ Departamento de Fisica Teorica C-15, Universidad Autonoma de Madrid, Madrid, Spain

${ }^{86}$ Institut für Physik, Universität Mainz, Mainz, Germany

${ }^{87}$ School of Physics and Astronomy, University of Manchester, Manchester, UK

${ }^{88}$ CPPM, Aix-Marseille Université and CNRS/IN2P3, Marseille, France

${ }^{89}$ Department of Physics, University of Massachusetts, Amherst, MA, USA

${ }^{90}$ Department of Physics, McGill University, Montreal, QC, Canada

${ }^{91}$ School of Physics, University of Melbourne, Victoria, Australia

92 Department of Physics, The University of Michigan, Ann Arbor, MI, USA

${ }^{93}$ Department of Physics and Astronomy, Michigan State University, East Lansing, MI, USA

94 (a) INFN Sezione di Milano, Milan, Italy; ${ }^{(b)}$ Dipartimento di Fisica, Università di Milano, Milan, Italy

95 B.I. Stepanov Institute of Physics, National Academy of Sciences of Belarus, Minsk, Republic of Belarus

${ }^{96}$ Research Institute for Nuclear Problems of Byelorussian State University, Minsk, Republic of Belarus

${ }^{97}$ Group of Particle Physics, University of Montreal, Montreal, QC, Canada

98 P.N. Lebedev Physical Institute of the Russian Academy of Sciences, Moscow, Russia

${ }^{99}$ Institute for Theoretical and Experimental Physics (ITEP), Moscow, Russia

${ }^{100}$ National Research Nuclear University MEPhI, Moscow, Russia

${ }^{101}$ D.V. Skobeltsyn Institute of Nuclear Physics, M.V. Lomonosov Moscow State University, Moscow, Russia 
102 Fakultät für Physik, Ludwig-Maximilians-Universität München, München, Germany

${ }^{103}$ Max-Planck-Institut für Physik (Werner-Heisenberg-Institut), München, Germany

${ }^{104}$ Nagasaki Institute of Applied Science, Nagasaki, Japan

105 Graduate School of Science and Kobayashi-Maskawa Institute, Nagoya University, Nagoya, Japan

106 (a) INFN Sezione di Napoli, Naples, Italy; ${ }^{(b)}$ Dipartimento di Fisica, Università di Napoli, Naples, Italy

${ }^{107}$ Department of Physics and Astronomy, University of New Mexico, Albuquerque, NM, USA

${ }^{108}$ Institute for Mathematics, Astrophysics and Particle Physics, Radboud University Nijmegen/Nikhef, Nijmegen,

The Netherlands

${ }^{109}$ Nikhef National Institute for Subatomic Physics and University of Amsterdam, Amsterdam, The Netherlands

${ }^{110}$ Department of Physics, Northern Illinois University, DeKalb, IL, USA

${ }^{111}$ Budker Institute of Nuclear Physics, SB RAS, Novosibirsk, Russia

112 Department of Physics, New York University, New York, NY, USA

113 Ohio State University, Columbus, OH, USA

${ }^{114}$ Faculty of Science, Okayama University, Okayama, Japan

${ }^{115}$ Homer L. Dodge Department of Physics and Astronomy, University of Oklahoma, Norman, OK, USA

116 Department of Physics, Oklahoma State University, Stillwater, OK, USA

117 Palacký University, RCPTM, Olomouc, Czech Republic

${ }^{118}$ Center for High Energy Physics, University of Oregon, Eugene, OR, USA

${ }^{119}$ LAL, Univ. Paris-Sud, CNRS/IN2P3, Université Paris-Saclay, Orsay, France

${ }^{120}$ Graduate School of Science, Osaka University, Osaka, Japan

${ }^{121}$ Department of Physics, University of Oslo, Oslo, Norway

122 Department of Physics, Oxford University, Oxford, UK

123 (a) INFN Sezione di Pavia, Pavia, Italy; ${ }^{(b)}$ Dipartimento di Fisica, Università di Pavia, Pavia, Italy

${ }^{124}$ Department of Physics, University of Pennsylvania, Philadelphia, PA, USA

${ }^{125}$ National Research Centre "Kurchatov Institute" B.P. Konstantinov Petersburg Nuclear Physics Institute, St. Petersburg, Russia

126 (a) INFN Sezione di Pisa, Pisa, Italy; ${ }^{\text {(b) }}$ Dipartimento di Fisica E. Fermi, Università di Pisa, Pisa, Italy

${ }^{127}$ Department of Physics and Astronomy, University of Pittsburgh, Pittsburgh, PA, USA

128 (a) Laboratório de Instrumentação e Física Experimental de Partículas-LIP, Lisbon, Portugal; ${ }^{(b)}$ Faculdade de Ciências,

Universidade de Lisboa, Lisbon, Portugal; ${ }^{(c)}$ Department of Physics, University of Coimbra,

Coimbra, Portugal; ${ }^{(d)}$ Centro de Física Nuclear da Universidade de Lisboa, Lisbon, Portugal; ${ }^{(e)}$ Departamento de Fisica, Universidade do Minho, Braga, Portugal; ${ }^{(\mathrm{f})}$ Departamento de Fisica Teorica y del Cosmos and CAFPE, Universidad de Granada, Granada, Spain; ${ }^{(\mathrm{g})}$ Dep Fisica and CEFITEC of Faculdade de Ciencias e Tecnologia, Universidade Nova de Lisboa, Caparica, Lisbon, Portugal

${ }^{129}$ Institute of Physics, Academy of Sciences of the Czech Republic, Prague, Czech Republic

${ }^{130}$ Czech Technical University in Prague, Prague, Czech Republic

${ }^{131}$ Charles University, Faculty of Mathematics and Physics, Prague, Czech Republic

${ }^{132}$ State Research Center Institute for High Energy Physics (Protvino), NRC KI, Protvino, Russia

133 Particle Physics Department, Rutherford Appleton Laboratory, Didcot, UK

134 (a) INFN Sezione di Roma, Rome, Italy; ${ }^{(b)}$ Dipartimento di Fisica, Sapienza Università di Roma, Rome, Italy

135 (a) INFN Sezione di Roma Tor Vergata, Rome, Italy; ${ }^{(b)}$ Dipartimento di Fisica, Università di Roma Tor Vergata, Rome, Italy

136 (a) INFN Sezione di Roma Tre, Rome, Italy; ${ }^{(b)}$ Dipartimento di Matematica e Fisica, Università Roma Tre, Rome, Italy

137 (a) Faculté des Sciences Ain Chock, Réseau Universitaire de Physique des Hautes Energies-Université Hassan II, Casablanca, Morocco; ${ }^{\left({ }^{b}\right)}$ Centre National de l'Energie des Sciences Techniques Nucleaires, Rabat, Morocco; ${ }^{\left({ }^{c}\right)}$ Faculté des Sciences Semlalia, Université Cadi Ayyad, LPHEA-Marrakech, Marrakech, Morocco; ${ }^{(d)}$ Faculté des Sciences, Université Mohamed Premier and LPTPM, Oujda, Morocco; ${ }^{(e)}$ Faculté des Sciences, Université Mohammed V, Rabat, Morocco

${ }^{138}$ DSM/IRFU (Institut de Recherches sur les Lois Fondamentales de l'Univers), CEA Saclay (Commissariat à l'Energie Atomique et aux Energies Alternatives), Gif-sur-Yvette, France

${ }^{139}$ Santa Cruz Institute for Particle Physics, University of California Santa Cruz, Santa Cruz, CA, USA

${ }^{140}$ Department of Physics, University of Washington, Seattle, WA, USA

${ }^{141}$ Department of Physics and Astronomy, University of Sheffield, Sheffield, UK 
142 Department of Physics, Shinshu University, Nagano, Japan

143 Department Physik, Universität Siegen, Siegen, Germany

${ }^{144}$ Department of Physics, Simon Fraser University, Burnaby, BC, Canada

${ }^{145}$ SLAC National Accelerator Laboratory, Stanford, CA, USA

146 (a) Faculty of Mathematics, Physics and Informatics, Comenius University, Bratislava, Slovak Republic; (b) Department of Subnuclear Physics, Institute of Experimental Physics of the Slovak Academy of Sciences, Kosice, Slovak Republic

147 (a) Department of Physics, University of Cape Town, Cape Town, South Africa; ${ }^{(b)}$ Department of Physics, University of

Johannesburg, Johannesburg, South Africa; ${ }^{(c)}$ School of Physics, University of the Witwatersrand, Johannesburg, South Africa

148 (a) Department of Physics, Stockholm University, Stockholm, Sweden; ${ }^{(b)}$ The Oskar Klein Centre, Stockholm, Sweden

149 Physics Department, Royal Institute of Technology, Stockholm, Sweden

${ }^{150}$ Departments of Physics and Astronomy and Chemistry, Stony Brook University, Stony Brook, NY, USA

${ }^{151}$ Department of Physics and Astronomy, University of Sussex, Brighton, UK

${ }^{152}$ School of Physics, University of Sydney, Sydney, Australia

${ }^{153}$ Institute of Physics, Academia Sinica, Taipei, Taiwan

${ }^{154}$ Department of Physics, Technion: Israel Institute of Technology, Haifa, Israel

155 Raymond and Beverly Sackler School of Physics and Astronomy, Tel Aviv University, Tel Aviv, Israel

${ }^{156}$ Department of Physics, Aristotle University of Thessaloniki, Thessaloníki, Greece

${ }^{157}$ International Center for Elementary Particle Physics and Department of Physics, The University of Tokyo, Tokyo, Japan

158 Graduate School of Science and Technology, Tokyo Metropolitan University, Tokyo, Japan

${ }^{159}$ Department of Physics, Tokyo Institute of Technology, Tokyo, Japan

160 Tomsk State University, Tomsk, Russia

${ }^{161}$ Department of Physics, University of Toronto, Toronto, ON, Canada

162 (a) INFN-TIFPA, Trento, Italy; ${ }^{\text {(b) }}$ University of Trento, Trento, Italy

163 (a) TRIUMF, Vancouver, BC, Canada; ${ }^{\text {(b) }}$ Department of Physics and Astronomy, York University, Toronto, ON, Canada

${ }^{164}$ Faculty of Pure and Applied Sciences, and Center for Integrated Research in Fundamental Science and Engineering,

University of Tsukuba, Tsukuba, Japan

165 Department of Physics and Astronomy, Tufts University, Medford, MA, USA

${ }^{166}$ Department of Physics and Astronomy, University of California Irvine, Irvine, CA, USA

167 (a) INFN Gruppo Collegato di Udine, Sezione di Trieste, Udine, Italy; ${ }^{\left({ }^{b}\right)}$ ICTP, Trieste, Italy; ${ }^{\left({ }^{c}\right)}$ Dipartimento di Chimica,

Fisica e Ambiente, Università di Udine, Udine, Italy

168 Department of Physics and Astronomy, University of Uppsala, Uppsala, Sweden

${ }^{169}$ Department of Physics, University of Illinois, Urbana, IL, USA

${ }^{170}$ Instituto de Fisica Corpuscular (IFIC), Centro Mixto Universidad de Valencia-CSIC, Valencia, Spain

171 Department of Physics, University of British Columbia, Vancouver, BC, Canada

172 Department of Physics and Astronomy, University of Victoria, Victoria, BC, Canada

${ }^{173}$ Department of Physics, University of Warwick, Coventry, UK

174 Waseda University, Tokyo, Japan

175 Department of Particle Physics, The Weizmann Institute of Science, Rehovot, Israel

${ }^{176}$ Department of Physics, University of Wisconsin, Madison, WI, USA

${ }^{177}$ Fakultät für Physik und Astronomie, Julius-Maximilians-Universität, Würzburg, Germany

${ }^{178}$ Fakultät für Mathematik und Naturwissenschaften, Fachgruppe Physik, Bergische Universität Wuppertal, Wuppertal, Germany

${ }^{179}$ Department of Physics, Yale University, New Haven, CT, USA

${ }^{180}$ Yerevan Physics Institute, Yerevan, Armenia

1811211 Geneva 23, Switzerland

182 Centre de Calcul de l'Institut National de Physique Nucléaire et de Physique des Particules (IN2P3), Villeurbanne, France

${ }^{183}$ Academia Sinica Grid Computing, Institute of Physics, Academia Sinica, Taipei, Taiwan

a Also at Department of Physics, King's College London, London, UK

${ }^{\mathrm{b}}$ Also at Institute of Physics, Azerbaijan Academy of Sciences, Baku, Azerbaijan

${ }^{\mathrm{c}}$ Also at Novosibirsk State University, Novosibirsk, Russia 
d Also at TRIUMF, Vancouver, BC, Canada

e Also at Department of Physics and Astronomy, University of Louisville, Louisville, KY, USA

${ }^{\mathrm{f}}$ Also at Physics Department, An-Najah National University, Nablus, Palestine

g Also at Department of Physics, California State University, Fresno CA, USA

h Also at Department of Physics, University of Fribourg, Fribourg, Switzerland

${ }^{i}$ Also at II Physikalisches Institut, Georg-August-Universität, Göttingen, Germany

j Also at Departament de Fisica de la Universitat Autonoma de Barcelona, Barcelona, Spain

${ }^{k}$ Also at Departamento de Fisica e Astronomia, Faculdade de Ciencias, Universidade do Porto, Porto, Portugal

${ }^{1}$ Also at Tomsk State University, Tomsk, Russia

${ }^{m}$ Also at The Collaborative Innovation Center of Quantum Matter (CICQM), Beijing, China

${ }^{\mathrm{n}}$ Also at Universita di Napoli Parthenope, Naples, Italy

${ }^{\circ}$ Also at Institute of Particle Physics (IPP), Victoria, BC, Canada

p Also at Horia Hulubei National Institute of Physics and Nuclear Engineering, Bucharest, Romania

q Also at Department of Physics, St. Petersburg State Polytechnical University, St. Petersburg, Russia

${ }^{\mathrm{r}}$ Also at Borough of Manhattan Community College, City University of New York, New York City, USA

${ }^{s}$ Also at Centre for High Performance Computing, CSIR Campus, Rosebank, Cape Town, South Africa

${ }^{\mathrm{t}}$ Also at Louisiana Tech University, Ruston, LA, USA

u Also at Institucio Catalana de Recerca i Estudis Avancats, ICREA, Barcelona, Spain

v Also at Graduate School of Science, Osaka University, Osaka, Japan

${ }^{w}$ Also at Fakultät für Mathematik und Physik, Albert-Ludwigs-Universität, Freiburg, Germany

${ }^{x}$ Also at Institute for Mathematics, Astrophysics and Particle Physics, Radboud University Nijmegen/Nikhef, Nijmegen, The Netherlands

y Also at Department of Physics, The University of Texas at Austin, Austin, TX, USA

${ }^{z}$ Also at Institute of Theoretical Physics, Ilia State University, Tbilisi, Georgia

aa Also at CERN, Geneva, Switzerland

ab Also at Georgian Technical University (GTU), Tbilisi, Georgia

ac Also at Ochadai Academic Production, Ochanomizu University, Tokyo, Japan

${ }^{\text {ad }}$ Also at Manhattan College, New York, NY, USA

${ }^{\text {ae }}$ Also at Departamento de Física, Pontificia Universidad Católica de Chile, Santiago, Chile

${ }^{\text {af }}$ Also at Department of Physics, The University of Michigan, Ann Arbor MI, USA

ag Also at School of Physics, Shandong University, Shandong, China

ah Also at Departamento de Fisica Teorica y del Cosmos and CAFPE, Universidad de Granada, Granada, Portugal

ai Also at Department of Physics, California State University, Sacramento, CA, USA

aj Also at Moscow Institute of Physics and Technology State University, Dolgoprudny, Russia

ak Also at Departement de Physique Nucleaire et Corpusculaire, Université de Genève, Geneva, Switzerland

${ }^{\text {al }}$ Also at Institut de Física d'Altes Energies (IFAE), The Barcelona Institute of Science and Technology, Barcelona, Spain

am Also at School of Physics, Sun Yat-sen University, Guangzhou, China

an Also at Institute for Nuclear Research and Nuclear Energy (INRNE) of the Bulgarian Academy of Sciences, Sofia, Bulgaria

ao Also at Faculty of Physics, M.V. Lomonosov Moscow State University, Moscow, Russia

ap Also at National Research Nuclear University MEPhI, Moscow, Russia

aq Also at Department of Physics, Stanford University, Stanford, CA, USA

${ }^{a r}$ Also at Institute for Particle and Nuclear Physics, Wigner Research Centre for Physics, Budapest, Hungary

as Also at Giresun University, Faculty of Engineering, Giresun, Turkey

${ }^{\text {at }}$ Also at CPPM, Aix-Marseille Université and CNRS/IN2P3, Marseille, France

au Also at Department of Physics, Nanjing University, Jiangsu, China

${ }^{a v}$ Also at University of Malaya, Department of Physics, Kuala Lumpur, Malaysia

${ }^{\text {aw }}$ Also at Institute of Physics, Academia Sinica, Taipei, Taiwan

${ }^{\text {ax }}$ Also at LAL, Univ. Paris-Sud, CNRS/IN2P3, Université Paris-Saclay, Orsay, France

* Deceased 